\title{
Aptamer-based detection of fumonisin B1: A systematic comparison with conventional and other novel methods
}

\author{
Vicente Antonio Mirón-Mérida ${ }^{1, *}$, Yun Yun Gong ${ }^{1}$, Francisco M. Goycoolea ${ }^{1, *}$ \\ 1 School of Food Science and Nutrition, University of Leeds, Leeds LS2 9JT, UK; Y.Gong@leeds.ac.uk \\ (Y.Y.G) \\ * Correspondence: fsvamm@leeds.ac.uk (V.A.M.M); F.M.Goycoolea@leeds.ac.uk (F.M.G.)
}

\begin{abstract}
Mycotoxin contamination is a current issue affecting several crops and processed products worldwide. Among the diverse mycotoxin group, fumonisin B1 (FB1) has become a relevant compound because of its adverse effects in the food chain. Conventional analytical methods previously proposed to quantify FB1 comprise LC-MS, HPLC-FLD and ELISA, while novel approaches integrate different sensing platforms and fluorescently labelled agents in combination with antibodies. Nevertheless, such methods could be expensive, time-consuming and require experience. Aptamers (ssDNA) are promising alternatives to overcome some of the drawbacks of conventional analytical methods, their high affinity through specific aptamer-target binding has been exploited in various designs attaining favorable limits of detection (LOD). So far, two aptamers specific to FB1 have been reported, and their modified and shortened sequences have been explored for a successful target quantification. In this critical review spanning the last eight years, we have conducted a systematic comparison based on principal component analysis of the aptamerbased techniques for FB1, compared with chromatographic, immunological and other analytical methods. We have also conducted an in-silico prediction of the folded structure of both aptamers under their reported conditions. The potential of aptasensors for the future development of highly sensitive FB1 testing methods is emphasized.
\end{abstract}

Keywords: Biosensors; mycotoxins; fumonisin B1; aptamers; aptasensors; analytical methods

\section{Introduction}

Mycotoxins are hazardous secondary metabolites produced by fungi, mainly Alternaria, Aspergillus, Claviceps, Fusarium and Penicillium. These biotic compounds act as hazards towards vertebrates, causing diseases when ingested, inhaled, or through skin contact. Some infectious processes, for instance, mycotoxicosis, take place after metabolization and accumulation of mycotoxins in several organs and tissues, due to immediate and progressive consumption of different contaminated food commodities [1], namely cereals, cocoa, coffee, fruit juices, milk and dairy, vegetable oils, beer, dried fruits, nuts, spices and their derived products. Multiple food matrices have been considered for the mitigation of toxin contamination [2], as mycotoxin occurrence takes place at different stages of the food chain, including field handling, storage and subsequent steps.

Exposure to mycotoxins is more likely to arise in regions with scarce methods for manipulation and storage of food products and can be related to other conditions such as malnutrition, limited regulations, and lack of protection for exposed groups [3]. Likewise, high-income countries are not exempt from mycotoxin occurrence, especially those importing agricultural and processed products from developing economies. As shown in Figure 1, there has been an increasing number of mycotoxin notifications in the last five years for the European Union (EU), whereas the United Kingdom (UK) has maintained a regular number of incidences, mostly identified through alerts, and border rejections of food and feed from EU member and non-member countries. To date, products such as peanuts, pistachios, hazelnuts, groundnuts, almonds, nutmeg, chilies, maize and dried figs are the most recurrent commodities exhibiting mycotoxin contamination; with a greater incidence in goods from Africa, South Asia, South America, China, USA and the Middle East [4]. 


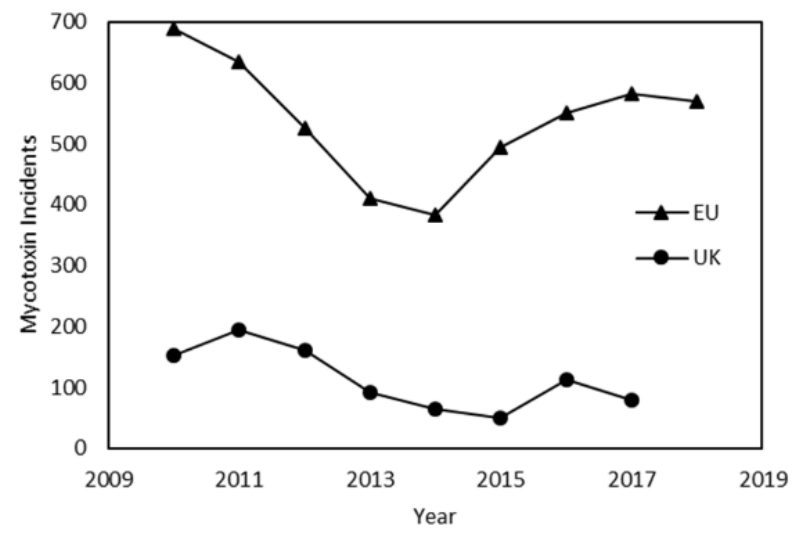

Figure 1. Number of mycotoxin notifications per year in the EU and the UK. Data based on the available Rapid Alert System for Food and Feed 2010-2018 by the European Commission [4] and the Incidents Annual Report 2010-2017 by the Food Standards Agency [184]

\subsection{Fumonisin B1}

Fumonisins are usually small alkyl amines containing two hydroxyl esterified propane tricarboxylic acids (tricarballylic acid), which are linked to adjacent carbons (Figure 2) [5]. When substituted in up to seven "R" side chains, the fumonisin aliphatic backbone serves as the basic structural unit for the conformation of different analogues. Existing fumonisin analogues can be classified in series $A, B, C$ and $P$, where group $B$ is the most abundant in nature [6]. Understanding the structure of fumonisins is critical when selecting and refining some quantification methods.

a)

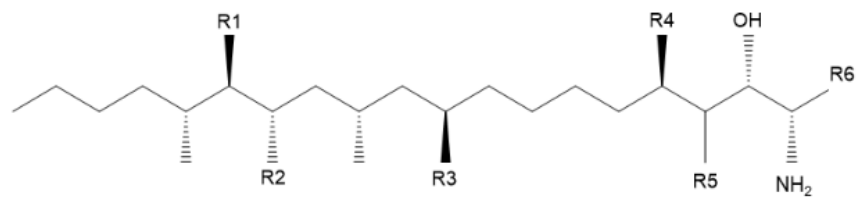

b)<smiles>COC(=O)CC(CC(=O)O)C(=O)O</smiles>

c)

\begin{tabular}{|c|c|c|c|c|c|c|}
\hline Fumonisin & R1 & R2 & R3 & R & R5 & R6 \\
\hline $\mathrm{FB}_{1}$ & TCA & TCA & $\mathrm{OH}$ & $\mathrm{OH}$ & & $\mathrm{CH}_{3}$ \\
\hline $\mathrm{FB}_{2}$ & TCA & TCA & $\mathrm{H}$ & $\mathrm{OH}$ & $\mathrm{H}$ & $\mathrm{CH}$ \\
\hline $\mathrm{FB}_{3}$ & TCA & TCA & $\mathrm{OH}$ & $\mathrm{H}$ & $\mathrm{H}$ & $\mathrm{CH}_{3}$ \\
\hline $\mathrm{FB}_{4}$ & TCA & TCA & $\mathrm{H}$ & $\mathrm{H}$ & $\mathrm{H}$ & $\mathrm{CH}_{3}$ \\
\hline Iso- $\mathrm{FB}$ B1 & TCA & TCA & $\mathrm{OH}$ & $\mathrm{H}$ & $\mathrm{OH}$ & $\mathrm{CH}_{3}$ \\
\hline FBK1 & & TCA & $\mathrm{OH}$ & $\mathrm{OH}$ & $\mathrm{H}$ & $\mathrm{CH} 3$ \\
\hline PHFB1a & TCA & $\mathrm{OH}$ & $\mathrm{OH}$ & $\mathrm{OH}$ & $\mathrm{H}$ & $\mathrm{CH} 3$ \\
\hline PHFB1b & $\mathrm{OH}$ & TCA & $\mathrm{OH}$ & $\mathrm{OH}$ & $\mathrm{H}$ & $\mathrm{CH} 3$ \\
\hline HFB1 & $\mathrm{OH}$ & $\mathrm{OH}$ & $\mathrm{OH}$ & $\mathrm{OH}$ & $\mathrm{H}$ & $\mathrm{CH}_{3}$ \\
\hline $\mathrm{FC} 1$ & TCA & TCA & $\mathrm{OH}$ & $\mathrm{OH}$ & $\mathrm{H}$ & $\mathrm{H}$ \\
\hline $\mathrm{FC} 3$ & TCA & TCA & $\mathrm{OH}$ & $\mathrm{H}$ & H & H \\
\hline $\mathrm{FC4}$ & TCA & TCA & $\mathrm{H}$ & H & $\mathrm{H}$ & $\mathrm{H}$ \\
\hline |soFC1 & TCA & TCA & $\mathrm{OH}$ & $\mathrm{H}$ & $\mathrm{OH}$ & $\mathrm{H}$ \\
\hline $\mathrm{OH}-\mathrm{FC} 1$ & TCA & TCA & $\mathrm{OH}$ & $\mathrm{OH}$ & $\mathrm{OH}$ & $\mathrm{H}$ \\
\hline
\end{tabular}

Figure 2. Structure representation of (a)the general fumonisin backbone, (b)tricarballylic acid (TCA) and (c)a list of alkyl amine fumonisins (c) [6]

Fumonisins B1 and B2 were initially studied and isolated from Fusarium verticillioides, formerly known as Fusarium monoliforme. They were discovered during the investigation of compounds responsible for leukoencephalomalacia, toxicity and hepatocarcinogenicity in some animal species [6]. Early studies reported the main role of $\mathrm{F}$. verticillioides in the production of FB1, FB2, FB3 (isoFB2), FB4, FA1, FA2 and FC1 [7, 8, 9, 10], when cultivated in liquid cultures and solid matrices (maize). However, depending on the host crop and growth media, fumonisins can be generated by other fungal species such as Alternaria alternata on potato dextrose agar [11], stationary cultures of Aspergillus niger producing FB6 and FB2 [12, 13], and some strains of Tolypocladium 
cylindrosporum, T. geodes and T. inflatum which developed fumonisins in high sugar media, when incubated at $25-30^{\circ} \mathrm{C}[14]$.

\subsection{Effects of fumonisin B1 on health}

Classified as group 2B hazard, fumonisins B1 and B2, are possible carcinogenic to humans [15]. Fumonisin B1 causes multiple effects on different species, its toxicity was first related to the disruption of sphingolipid metabolism, as this mycotoxin inhibits ceramide synthase, which leads to both an increase in sphinganine and a decrease in complex sphingolipids, and further cell death observed in pig kidney cells $[16,17]$. Notwithstanding this frequent assumption, studies on the protective role of liver $\mathrm{X}$ receptor (LXR) on FB1-caused hepatotoxicity implied the presence of different pathways [18].

Another mechanism triggered by FB1 is oxidative stress, where FB1 reduces mitochondrial and cellular respiration and increases the production of reactive oxygen species, as observed in rat astrocytes and human neuroblastoma cells [19]. In the same way, FB1 reduced growth of pig iliac endothelial cells and their barrier functions, while decreased the activities of some enzymes with antioxidant effects and enhanced the formation of lipid peroxidation compounds [20]. Exposure to fumonisin could also induce epigenetic changes such as DNA methylation and hypomethylation in rat glioma cells and human intestinal and hepatoma cells [19]. Apart from neurotoxicity, hepatotoxicity, nephrotoxicity, and carcinogenicity, FB1 has also been studied in corneal infections, due to its ability to form Langmuir monolayers on liquid surfaces [21]. Besides, some geographical studies have correlated the prevalence of esophageal cancer in humans with the presence of FB1 and FB2 in regional crops [22, 23].

In addition, adverse effects from fumonisins in human health were reported for Mexican American women living in the border region between Mexico and Texas, where fumonisin exposure was associated with neural tube defects [24]. Fumonisin B1 occurrence in Tanzania was reported in breastfeeding with contaminated milk as a current issue among children under six months of age [25]; elevated levels of dietary fumonisin were likewise related to inhibition of ceramide synthase in women from Guatemala [26], whose consumption of contaminated maize was detected in their high urinary fumonisin levels [27]. Other studies conducted in Tanzania have demonstrated the main role of fumonisin in underweight children due to breastfeeding and weaning within the first 36 months of age [28] as well as the high impact of substituting breastfeeding on the infant mycotoxin exposure [25]. Even though fumonisin B1 is not as prioritized as other mycotoxins, single exposure to it and its combination with other mycotoxins such as aflatoxins, represent an issue that needs to be addressed in deep, due to its common occurrence.

\subsection{Fumonisin occurrence in food commodities and its worldwide regulation}

The Food and Agriculture Organization (FAO) of the United Nations trough the worldwide regulations for mycotoxins in food and feed, indicated that by 2003 only 99 countries had regulations in place focused on mycotoxins. Additionally, the extent of those actions covered a brief group of different toxins among continents. As it can be noticed from Figure 3, the regulations for fumonisins in food and feed are established on either the sum of fumonisins type B1+B2+B3, B1+B2, or as total content of FB1 [29]. 


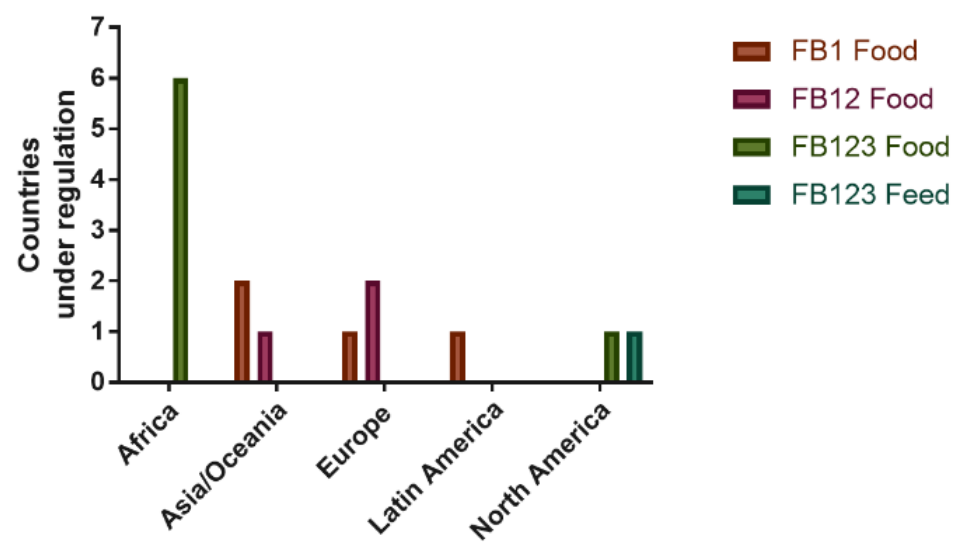

Figure 3. Countries regulating fumonisin in food and feed worldwide [29]

The number of countries under fumonisin regulations is equivalent for Europe and Asia/Oceania. On the other hand, the North America region has a noticeable approach by the United States, where limits for mycotoxins are targeted not only in food, but in feed. Based on the FAO controls, Africa was overall the less active region in enforcing mycotoxin regulations, particularly for any type of fumonisin. Paradoxically, though perhaps not surprising, the highest incidence of mycotoxins in food and feed occurs in Africa [30], however since 2011 a control for aflatoxin and fumonisin was established by the East African Community (EAS), whose scope included the six member countries, with a potential application on the trade activities by the twenty COMESA member states (Table 1). Furthermore, Latin America possesses a gap in recognizing fumonisins as an important group of hazardous compounds [29].

The growth of fumonisin producing fungal species has been reported on corn seedlings, grits, meal and flour, tomato leaves, seedlings and rice [5, 9, 31, 32, 33] as well as some dried samples comprising coffee beans and vine fruits [34, 35]. Some Fusarium species can produce fumonisins in media based on rice, oat, carrots and malt. In contrast, A. niger requires low water activity media and products with high sugar content [12, 35]. Maximum levels of fumonisins in both food and feed are shown in Table 1. As previously mentioned, cereals, rice and maize food and feed products are the most common targeted commodities for possible fumonisin outbreaks. Understanding the maximum values established by regulation, along with the expected contamination levels for distinct samples, is crucial during the design of conventional and novel quantification methods. Also, it is necessary to know the scope and applicability of each technique. The focus of this systematic comparison centers in these aspects by reflecting the state of the art in the field since 2012.

\section{Methods}

\subsection{Systematic comparison}

For this systematic comparison a screening was made from results obtained after searching the words "fumonisin + aptamer" and "FB1 + aptamer" in Scopus $(28,12)$, Web of science $(28,14)$, and Google Scholar (4, 32); as well as papers containing the specific DNA sequences. As indicated in Scheme 1, from the 29 relevant papers, 27 biosensors were identified and compared with other conventional methods for FB1 detection in terms of their limit of detection (LOD), assay time, and assay preparation time. The data were plotted in GraphPad Prism 7 to show the evolution and relation of such parameters throughout the years. 
Table 1. Maximum permitted levels $(\mu \mathrm{g} / \mathrm{kg})$ of fumonisins in food and feed set by different organizations ${ }^{1}$

\begin{tabular}{|c|c|c|c|c|c|}
\hline Commodity & $\begin{array}{c}\text { Maximum } \\
\text { Level }(\mu \mathrm{g} / \mathrm{kg})\end{array}$ & Type & Authority & $\begin{array}{l}\text { Regulatory } \\
\text { Framework }\end{array}$ & Country \\
\hline Raw maize grain & 4000 & B1, B2 & FAO, WHO & $\begin{array}{l}\text { CODEX STAN } \\
193-1995\end{array}$ & International trade \\
\hline Maize flour and maize meal & 2000 & B1, B2 & $\mathrm{FAO}, \mathrm{WHO}$ & $\begin{array}{l}\text { CODEX STAN } \\
193-1995\end{array}$ & International trade \\
\hline Unprocessed maize (not for milling) & 4000 & B1, B2 & CEC & $\begin{array}{l}\text { (EC) No } \\
1126 / 2007\end{array}$ & EU \\
\hline $\begin{array}{l}\text { Maize, maize-based foods for direct human } \\
\text { consumption }\end{array}$ & 1000 & B1, B2 & CEC & $\begin{array}{l}\text { (EC) No } \\
1126 / 2007\end{array}$ & EU \\
\hline Maize-based breakfast cereals and snacks & 800 & B1, B2 & CEC & $\begin{array}{l}\text { (EC) No } \\
1126 / 2007\end{array}$ & EU \\
\hline $\begin{array}{l}\text { Processed maize-based foods and baby } \\
\text { foods (Infants and young children) }\end{array}$ & 200 & B1, B2 & CEC & $\begin{array}{l}\text { (EC) No } \\
1126 / 2007\end{array}$ & EU \\
\hline $\begin{array}{l}\text { Milling fractions according to size (500 } \\
\text { micron) and CN code } 19041010\end{array}$ & $1400-2000$ & B1, B2 & CEC & $\begin{array}{l}\text { (EC) No } \\
1126 / 2007\end{array}$ & EU \\
\hline Maize and processed products & 1000 & B1, B2 & $\mathrm{MH}$ & BG1 & Bulgaria \\
\hline Maize, rice & 1000 & & $\mathrm{MPH} / \mathrm{INHA}$ & CU1 & Cuba \\
\hline Cereals \& cereal products & 1000 & B1 & DGCCRF & FR1 & France \\
\hline Maize & 1000 & B1, B2 & $\begin{array}{l}\text { ISIRI, } \\
\mathrm{MOH}\end{array}$ & IR1 & Iran \\
\hline Corn \& corn products & Not given & B1 & SG1 & AVA & Singapore \\
\hline Maize & 1000 & B1, B2 & $\mathrm{CH} 1$ & OFCACS & Switzerland \\
\hline Maize products & $\begin{array}{l}\text { According to the } \\
\text { result of risk } \\
\text { assessment }\end{array}$ & B1 & - & - & Taiwan \\
\hline $\begin{array}{l}\text { Degermed dry milled corn products (e.g. } \\
\text { flaking grits, corn grits, corn meal, corn flour } \\
\text { with fat content of }<2.25 \% \text {, dry weight basis) }\end{array}$ & 2000 & $\begin{array}{l}\text { B1, B2, } \\
\text { B3 }\end{array}$ & US4, US5 & FDA & USA \\
\hline Cleaned corn intended for popcorn & 3000 & $\begin{array}{l}\text { B1, B2, } \\
\text { B3 }\end{array}$ & US4, US5 & FDA & USA \\
\hline $\begin{array}{l}\text { Whole of partially degermed dry milled corn } \\
\text { products (e.g. flaking grits, corn grits, corn } \\
\text { meal, corn flour with fat content of } \geq 2.25 \% \text {, } \\
\text { dry weight basis); dry milled corn bran; } \\
\text { cleaned corn intended for masa production }\end{array}$ & 4000 & $\begin{array}{l}\mathrm{B} 1, \mathrm{~B} 2, \\
\mathrm{~B} 3\end{array}$ & US4, US5 & FDA & USA \\
\hline $\begin{array}{l}\text { Corn and corn by-products intended for } \\
\text { equids and rabbits }\end{array}$ & 5000 & $\begin{array}{l}\mathrm{B} 1, \mathrm{~B} 2, \\
\mathrm{~B} 3\end{array}$ & US4, US5 & FDA & USA \\
\hline $\begin{array}{l}\text { Corn and corn by-products intended for } \\
\text { swine and catfish }\end{array}$ & 20000 & $\begin{array}{l}\mathrm{B} 1, \mathrm{~B} 2, \\
\mathrm{~B} 3\end{array}$ & US4, US5 & FDA & USA \\
\hline $\begin{array}{l}\text { Corn and corn by-products intended for } \\
\text { breeding ruminants, breeding poultry and } \\
\text { breeding mink (includes lactating dairy cattle } \\
\text { and hens laying eggs for human } \\
\text { consumption) }\end{array}$ & 30000 & $\begin{array}{l}\text { B1, B2, } \\
\text { B3 }\end{array}$ & US4, US5 & FDA & USA \\
\hline $\begin{array}{l}\text { Ruminants } \geq 3 \text { months old being raised for } \\
\text { slaughter and mink being raised for pelt } \\
\text { production }\end{array}$ & 60000 & $\begin{array}{l}\text { B1, B2, } \\
\text { B3 }\end{array}$ & US4, US5 & FDA & USA \\
\hline Poultry being raised for slaughter & 100000 & $\begin{array}{l}\text { B1, B2, } \\
\text { B3 }\end{array}$ & US4, US5 & FDA & USA \\
\hline $\begin{array}{l}\text { All other species or classes of livestock and } \\
\text { pet animals }\end{array}$ & 10000 & $\begin{array}{l}\text { B1, B2, } \\
\text { B3 }\end{array}$ & US4, US5 & FDA & USA \\
\hline Maize grains/ Millet flour & 2000 & Fumonisin & EAC & EAS & $\begin{array}{l}\text { Burundi, Kenya, Rwanda, } \\
\text { South Sudan, Tanzania, } \\
\text { Uganda. }\end{array}$ \\
\hline \multicolumn{6}{|c|}{ 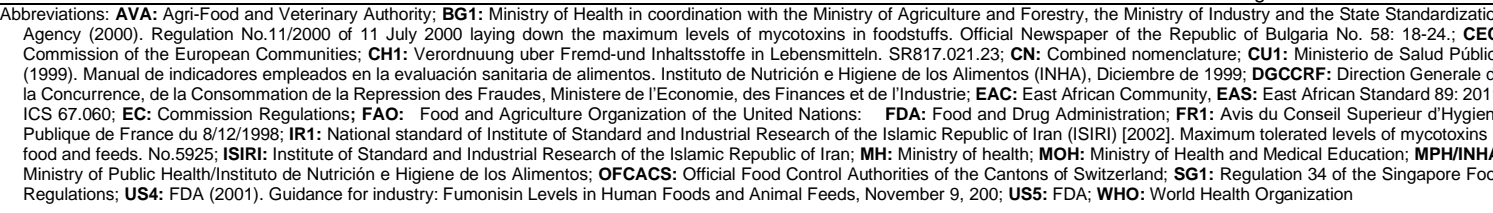 } \\
\hline
\end{tabular}

\subsection{Principal component analysis}

The aptamer-based biosensors for FB1 detection and several conventional and novel methods published since 2012 (publication year of the first aptasensor), were combined in a principal component analysis, performed in Minitab 15 Statistical Software. Before the application of the correlation matrix, all data were treated according to the following equations:

$$
L O D t=\frac{L O D \max }{L O D} \quad A T t=\frac{A T \max }{A T} \quad A P t=\frac{A P \max }{A P}
$$

Where LODt, ATt and APt are the treated limit of detection, assay time and assay preparation time, respectively; LODmax and ATmax are the maximum limit of detection and maximum assay time for all the data in this comparison (since 2012), equal to $3200 \mu \mathrm{g} / \mathrm{L}$ [90] and $720 \mathrm{~min}$ [166] respectively. The assay preparation time was calculated by adding the reported times for sample extraction, 
synthesis of nanoparticles, support treatment, and array assembling. The maximum preparation time per assay was calculated as $12900 \mathrm{~min}$ [151]. This mathematical treatment allowed to determine the correlation of the maximum values to the most sensitive, fast and therefore, effective methods.

\subsection{DNA folding}

The DNA folding forms of the four existing aptamers were predicted with mfold Web Server according to their reported folding conditions.

\section{Conventional and novel methods for mycotoxin identification}

Typical methods for the identification of mycotoxins in food samples incorporate compound separation principles for quantification through TLC, HPLC, and LC-MS. At the same time, some commercial immunoassays optimized the use of antibodies for mycotoxin quantification. However, most of them utilize expensive and sophisticated equipment for time-consuming assays that are required to be performed by skilled operators, as they utilize complex elements and instruments [36, 37]. Novel approaches including optical, electromagnetic, electrochemical and surface-sensitive techniques (e.g., surface plasmon resonance, ion-selective field-effect transistors, surfacedenhanced Raman spectroscopy) along with aptamer-based techniques, have been developed and found to exhibit comparable and even higher sensitivities in than that of conventional procedures.

Based on Tables 2-5, the LODs of different reported methods were plotted against their total assay times, as reflected on Figure 4a. The assay time was calculated from either the divulged times at either the injection step in chromatography, or the incubation between the antibody/aptamer/recognition region with its corresponding target molecule. This consideration excluded any pre-treatment, extraction steps and particle fabrication, as those phases were part of the assay preparation time (Fig 4b). The shortest response time for the analysis of extracted samples was achieved in seconds to minutes, when using Surface-enhanced Raman spectroscopy [140]. Nevertheless, some sensors qualified as fast required overnight steps and long incubation times for the whole system arrangement, especially when the synthesis of nanoparticles and drying phases were required. Assay times below ten minutes were achieved through chromatographic, immunoassays, and some innovative methods, nonetheless the more sensitive assays were secured with aptamer-based biosensors [170, 177, 200], immunosensors with carbon nanotubes [101, 109], and molecularly imprinted polymer nanoparticles (MIPs) [191], as indicated in Figures 4a and 4c.

In addition to high specificity, the combination of minimum assay times with low limit of detections is ideal for an appropriate quantification technique. Nonetheless, an increase in the assay preparation time can complicate the achievement of on-site/point of care analysis and compromise the reproducibility. Even though there is high sensitivity achieved through aptasensors, such DNA-based techniques along with some immunoassays, entail long assay times with extended preparation time, due to incubation and platform preparation, respectively (Figure 4b). In those cases, the final response was normally measured as either a fluorescent or a colorimetric signal. Figure 4c portrays the LODs accomplished per year, where it can be noted that ongoing research is still focused on developing chromatographic techniques and immunoassays. 
a)

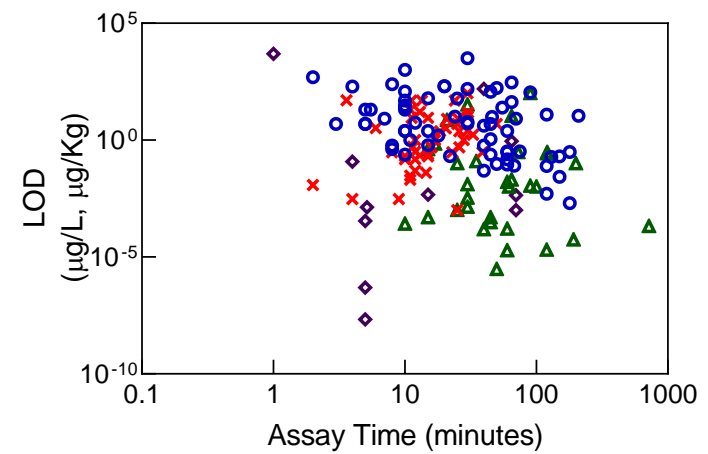

b)

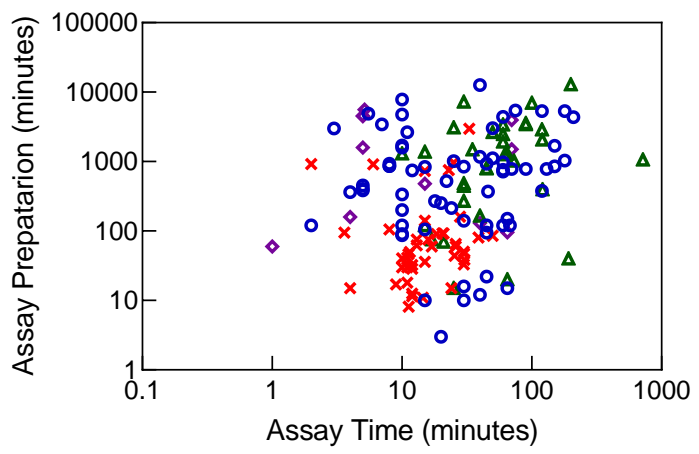

c)

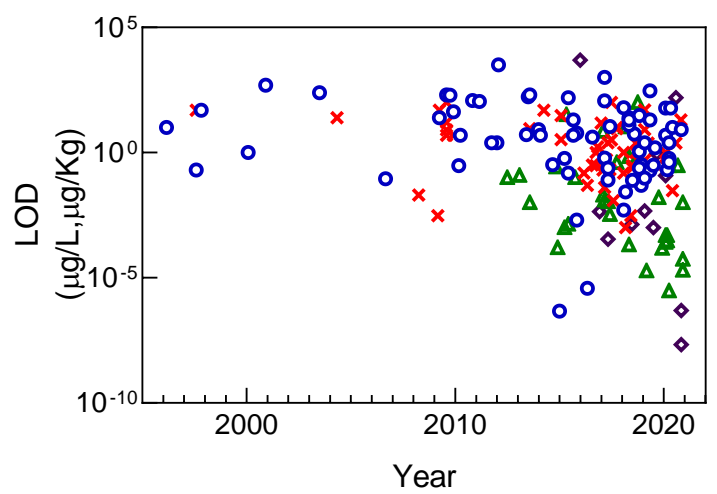

Figure 4. Relation of the assay time with (a) the limit of detection (LOD) and (b) assay preparation time for the approaches reported since 2012, (c) and LODs achieved over time through different methods (O: Immunologic, $\mathrm{X}$ :Chromatographic, $\Delta$ : Aptamer-based, $\diamond$ : Other)

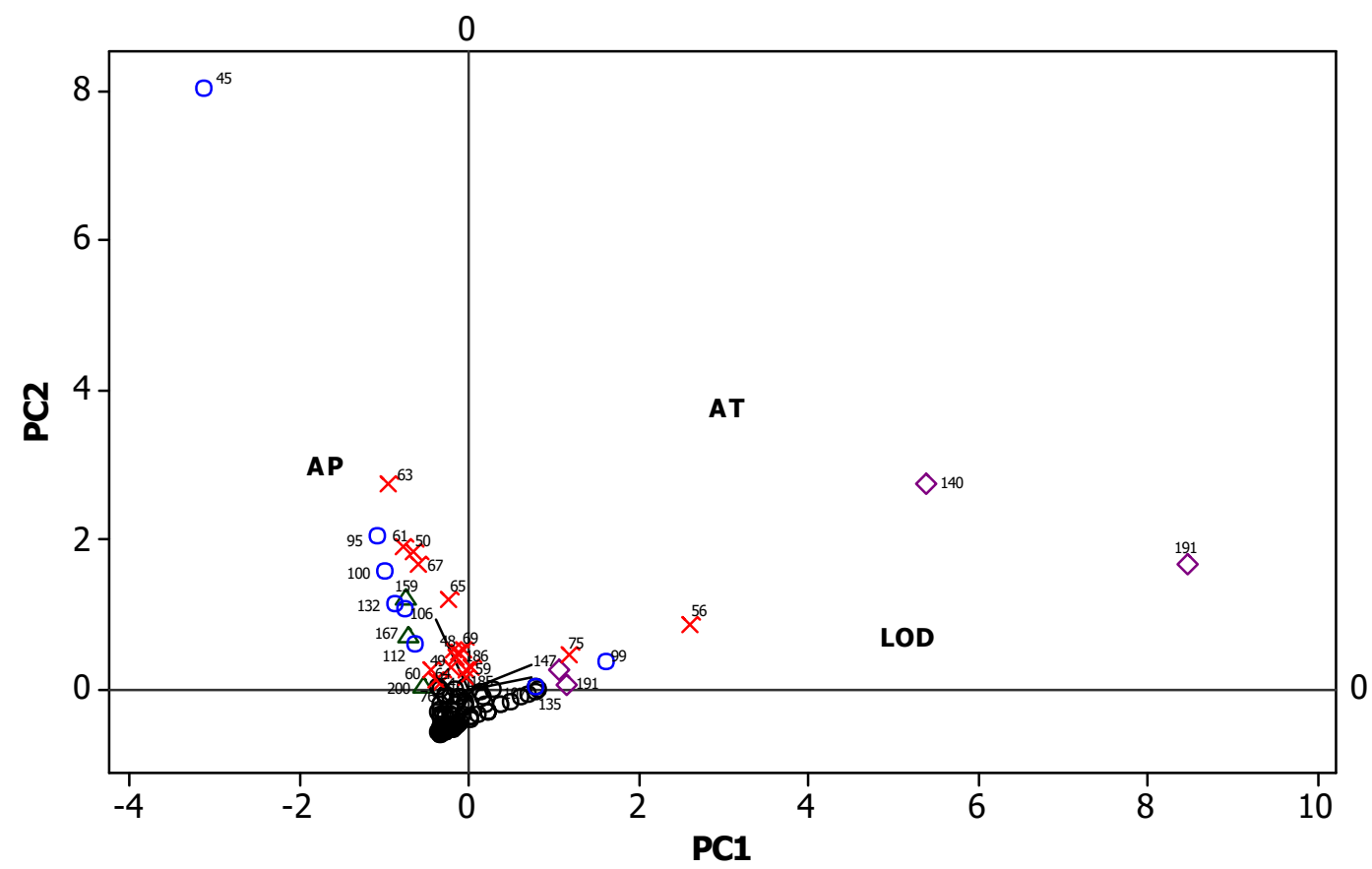

Figure 5. Principle component analysis for the correlation of aptasensors and conventional methods reported from 2012 to the lowest detection limits (LOD), assay time (AT) and assay preparation time (AP). (O: Immunologic, X: Chromatographic, $\Delta$ : Aptamer, $\diamond:$ Other). The numbers correspond to the correlated references from Tables 2-5. 
Although, over the last five years there has been an improvement on the detection limits of some protocols, especially for immunoassays whose LODs have reached the picogram scale, most of the new chromatographic and antibody-based methods still quantify values comparable to earlier findings. Conventional assays with the highest sensitivity have included electrochemical designs, electrochemiluminescent quantifications, and MS detection (Table 2-3). Of note, fluorescent, colorimetric and electrochemical aptamer-based sensors reported over the last three years, accomplished relevant LODs with a promising tendency (Figure 4).

Despite the fact that the use of antibodies with electrochemical readouts was advantageous for achieving some of the lowest LODs for fumonisin B1, equivalent to $4.6 \mathrm{e}-7$ and $3.7 \mathrm{e}-6 \mu \mathrm{g} / \mathrm{L}[101,109]$, these immunosensors were not included in the principal component analysis (PCA), as no assay time was reported in either case. Hence, as indicated in Figure 5, LC-MS [56,75], immunoassays with optical $[99,107,135]$, Raman (due to its quick procedure) [140], fluorescent readouts [147] and electrochemical MIPs [191] were correlated to the combination of low LODs with short assay times. However, such statistical analysis did not show the advantages of aptamer-based methods, which was also observed on the correlation of short assay preparation times with LC-MS, immunologic and only three aptasensors [159, 167, 200]. This was shown by PCA, where the main drawbacks from aptamer-based sensors for FB1 was their long assay and assay preparation times denoted by is absence of correlation in both components when compared to other methods.

\subsection{Chromatographic detection of fumonisin B1}

Together with immunoassays, chromatographic methods for the quantification of mycotoxins, have been widely studied and optimized for the analysis of several food products as indicated in Table 2. Initial chromatographic techniques were focused on the exclusive quantification of fumonisin in corn, through the analysis of either MS/MS or fluorescence signals. Following analysis confirmed the good correlation of maize-based products expenditure with FB1 levels in human urine [40]; which consolidated its utilization as a relevant biomarker, as a portion of ingested FB1 is excreted in urine [44].

The detection of fumonisins is limited by its absence of fluorescence; therefore, the introduction of a chromophore for the derivatization of the amino groups within fumonisin is always required [179]. Initial derivatization procedures utilized maleic anhydride derivatives and fluorescamine $[7,180]$. Nevertheless, more sensitive detection procedures introduced but still utilize pre-column derivatization with o-phthaldialdehyde [39, 42, 46, 181, 182], naphthalene-2,3-dicarboxaldehyde [41], and the quick and stable (9-fluorenylmethyl) chloroformate (FMOC) $[55,183]$.

Fluorescence detectors are restricted for the individual quantification of $\mathrm{FB} 1[38,46,47]$, the sum of FB1, FB2, FB3 [41] or the separate determination of up to three group B fumonisins [42, 55]. On the other hand, one of the main advantages of mass spectrometry detectors is the possibility of performing multiplex analysis, not only for different mycotoxins $[48,49,50,53,54,57,58,59,60,61$, $64,65,66,67,71,72,73,74,77,79,80,187]$, but also when combined with varied metabolites. Growth regulators, antibiotics, pesticides [51, 70,75], and other fungal metabolites [63,68], were simultaneously identified in analysis capable of assessing up to 74 and 90 compounds $[62,78]$. Mass spectrometry has also been used for assessing mycotoxin transfer from feed to organs and tissue in poultry [80]

The importance of novel analytical methods relies on the low sensitivities achieved within a relatively short detection time. The speed of mass spectrometry (ESI+) signals, was early proven to reduce the sole determination of FB1 in bovine milk to 4 minutes [43], with a half-fold time reduction on more recent assays for pig samples (plasma, urine, feces) [56]. Its limits of detection have reached $0.003 \mu \mathrm{g} / \mathrm{kg}[43,65]$ for animal (pig) and food samples (corn meal), and $0.001 \mu \mathrm{g} / \mathrm{L}$ in human urine [73]. Notwithstanding the excellent performance of conventional analytical methods, some disadvantages are related to sample pre-treatment including long extraction steps with further purification protocols, as well as method optimization of the chromatographic separation, derivatization or internal standard addition, along with its corresponding validation method. For instance, a single drying step could add two days to the total assay preparation time [53].

Sample clean-up is a key step for reducing matrix effect, where strong anion exchange (SAX) columns have been utilized as cheaper clean up cartridges in LC-MS detection, with recoveries of up 
to 86.6 and $106 \%$ for human hair [47] and piglet urine [56] respectively. In a similar way, immunoaffinity columns (IAC) have been proven to attain maximum recoveries of $109 \%$ for FMOCderivatized cornmeal samples [55], and $90 \%$ in rice analyzed by LC-MS [75]. The specificity of antibodies in IAC also allowed the successful LC-MS analysis of FB1 in complex samples, such as milk [43], human urine [44] and chicken tissue [80] with peak recoveries of $88.4 \%, 99.1 \%$ and 95 $102 \%$ respectively. Lower recoveries were found for the determination of OPA-derivatized FB1 in maize $(68.5 \%)$, rice $(72.4 \%)$, sorghum $(75.6 \%)$ and wheat $(69.4 \%)$ extracts [42]. Nonetheless, IACs increase the total assay cost, since they could account for double or triple the price of SAX cartridges, with highly comparable performance. Besides, IACs have a limitation on the variability of analytes and could promote interaction with the matrix constituents [77]. In both cases (SAX and IAC) the total analysis time is enlarged by the conditioning, loading, washing, elution, evaporation, and reconstitution steps.

Some novel developments incorporated magnetic nanoparticles for the sorption and concentration of mycotoxins, promoting a simultaneous clean-up and sensitivity enhancement in the overall method [59]. Nonetheless, even when the performance of patented commercial clean up columns allows their utilization in single [39] and multiple mycotoxin analysis, the adsorption procedure of recent products might impede the detection of FB1 and FB2 [53]. As a replacement, novel dispersants such as nano zirconia, have been found with high extraction efficiency of FB1 [65].

Alternatively, the QuEChERS method, initially developed for pesticides, was subsequently introduced for the dispersive solid-phase extraction (SPE) of FB1 [46], and further validated for its application in the multi-target analysis due to its lower cost, less time consumption, easy procedure $[50,52,63,185,186]$, as well as its availability in extraction kits [54] with satisfactory recoveries [60]. Likewise, sample preparation with a QuEChERS dispersive SPE was useful for minimization of matrix effects from beer, with a preconcentration step producing enhanced LODs [74]. In spite of being a favorable option for sugar reduction in the quantification of FB1 in oat, soy and rice beverages (extraction recoveries $80,82,85 \%$; matrix effect: 76, 63, 75\%) [61], a UPLC-MS/MS study of Alpinia oxyphylla revealed the unsatisfactory FB1 and FB2 recoveries from QuEChERS ( 50 \& 55\%) and hydrophilic-lipophilic balance (HLB) cartridges ( 65 \& 55\%) in comparison to solid-liquid extraction ( $80 \& 70 \%)$. Nevertheless, the three extraction methods exhibited a signal increase $(80-145 \%)$ due to matrix effect [64]. By contrast, recent studies in sugarcane juice proposed the use of HLB cartridges as an alternative to QuEChERS, due to its high recoveries of 98\% for FB1 [79].

Despite the expected disadvantages of the dilute and shoot method towards the complexity of some samples, which could affect the detector sensitivity and assay performance, when optimized, this procedure can be applied in the multi-target analysis of food samples without a clean-up phase $[61,68,70,75]$. For instance, a comparison between the efficiency of dilute and IAC methods revealed that, even when lower LODs and limits of quantification (LOQs) were obtained with the clean-up step ( 0.5 and 1.66 against 2.3 and $4.3 \mu \mathrm{g} / \mathrm{kg}$ ), a dilution procedure accomplished improved regression (0.9941), high recoveries (94-106\%) and reproducibility for FB1-spiked animal feed [77].

A similar situation was confirmed for matrix-match calibration $[45,49,70]$, and internal standard (IS) addition [48, 66, 77] where a clean-up step was not necessary to eliminate matrix effects and run accurate determinations. Yet, the use of specific IS and a validated method for a single matrix, could reduce the scope of the determination, and increase its final cost. Notwithstanding, some approaches proposed the application of the aforementioned procedures combined with clean up techniques and QuEChERS, for a greater method validation $[58,69,71,73]$. 
Table 2. Chromatographic determination of $\mathrm{FB} 1^{1}$

\begin{tabular}{|c|c|c|c|c|c|c|c|c|}
\hline Support & Method & Eluent & Measurement & $\begin{array}{c}\text { Assay } \\
\text { Time (min) }\end{array}$ & $\begin{array}{l}\text { Limit of } \\
\text { Detection }\end{array}$ & Sample & Fumonisin Type & Ref \\
\hline Wakosil $5 \mathrm{C} 18$ column & HPLC & $\begin{array}{l}\text { Acidified methanol and disodium phosphate }(80: 20 \mathrm{pH} \\
3.3)\end{array}$ & Fluorescence & 24 & $50 \& 100 \mu g / K g$ & Corn & $\begin{array}{c}\text { FB1, } \\
\text { FB2 FB3 }\end{array}$ & [38] \\
\hline 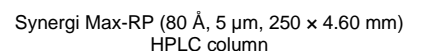 & HPLC & $\begin{array}{l}\text { Methanol//.1 M phosphate buffer }(77.23, \mathrm{v} / \mathrm{v}) \text { adjusted } \\
\text { to } \mathrm{pH} 3.35 \text { with concentrated orthophoshoric acid. }\end{array}$ & Fluorescence & - & $25 \mu \mathrm{g} / \mathrm{Kg}$ & $\begin{array}{l}\text { Corn kernels, tortillas and } \\
\text { masa }\end{array}$ & FB1 & [39] \\
\hline $\begin{array}{l}\text { Luna C18 column }(50 \times 4.6 \mathrm{~mm} \text { ID, } 5 \mu \mathrm{mm} \\
\text { Phenomenex) }\end{array}$ & LC-MS & $\begin{array}{l}\text { Water/acetonitrilefformic acid at } \\
\text { Wo }\end{array}$ & MS detection & 11 & $0.02 \mu \mathrm{g} / \mathrm{L}$ & Urine (Tortilla consumption) & FB1 & [40] \\
\hline Column C18 XTerra Waters narrow bore with a & LC & Acidified water \& methanol & MS/MS analysis & 50 & $5 \mu \mathrm{g} / \mathrm{kg}$ & Corn & $\mathrm{FBs}, \mathrm{HFBs}$ & [41] \\
\hline 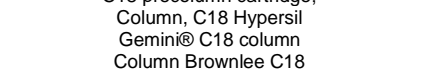 & $\begin{array}{l}\text { LC } \\
\text { HPLC } \\
\text { HPLC }\end{array}$ & $\begin{array}{l}\text { Acidified water \& acetonitrile } \\
\text { Methanol/water/acetic acid with ammonium acetate } \\
\text { Water-acetonitrile-acetic acid }\end{array}$ & $\begin{array}{l}\text { MS/MS analysis } \\
\text { MS/MS analysis } \\
\text { Fluorescence }\end{array}$ & $\begin{array}{l}13 \\
21\end{array}$ & $\begin{array}{c}16 \mu \mathrm{gg} / \mathrm{kg} \\
8 \mu \mathrm{kg} \\
100 \mu \mathrm{\mu g} / \mathrm{kg}\end{array}$ & $\begin{array}{l}\text { Corn } \\
\text { Corn } \\
\text { Corn }\end{array}$ & $\begin{array}{l}\text { HFBs } \\
\text { HFBS } \\
\text { FBs }\end{array}$ & $\left.\begin{array}{l}{[41]} \\
{[41]} \\
{[41]}\end{array}\right]$ \\
\hline Symmetry Spherisorb ODS2 C18 Column & HPLC & Methanol\& sodium dihydrogen phosphate & Fluorescence & 11.20 & $50 \mu \mathrm{\mu g} / \mathrm{kg}$ & Tunisian foods and feed & FB1, & [42] \\
\hline XTerra MS C18 column & $\begin{array}{l}\text { LC-MS- } \\
\text { MS }\end{array}$ & Acidified water:acetonitrile \& acetonitrile & MS-MS detection & $\begin{array}{l}<4 \mathrm{~min}(\mathrm{LC}- \\
\text { ESI-MSMSS } \\
\text { signal) }\end{array}$ & $0.003 \mu \mathrm{g} / \mathrm{kg}$ & Bovine Milk & FB1 & [43]. \\
\hline Luna C18 column & $\begin{array}{l}\text { LC-MS- } \\
\text { MS }\end{array}$ & Acidified water \& methanol & MS-MS detection & 25 & $5 \mu \mathrm{g} / \mathrm{L}$ & Urine & $\mathrm{FB} 1, \mathrm{FB} 2$ & [44] \\
\hline Agilent Zorbax Eclipse XDB C- 18 column & LC- & Acidified water \& methanol & MS & 15 & $9,6 \mu \mathrm{g} / \mathrm{kg}$ & Maize & $\mathrm{FB} 1, \mathrm{FB} 2$ & [45] \\
\hline Hypersil'TM ODS C18 Columns & $\begin{array}{l}\text { MSMS } \\
\text { HPLC }\end{array}$ & Acetonitrile \& sodium phosphate buffer & Fluorescence & $\begin{array}{c}\sim 13.5 \\
\text { (retention } \\
\text { time) }\end{array}$ & $50 \mu \mathrm{g} / \mathrm{kg}$ & Rice & FB1 & [46] \\
\hline Shimadzu C18 column & HPLC & Water/acetonitrile/acetic acid & Fluorescence & - tom & $30 \mu \mathrm{g} / \mathrm{kg}$ & Corn & FB1 & [47] \\
\hline Thermo Hypersil GOLD column & LC-MS & Acidified water \& acetonitrile & MS detection & 6 & $3.3 \mu \mathrm{g} / \mathrm{kg}$ & Human hair & $\mathrm{FB} 1$ & [47] \\
\hline $\begin{array}{l}\text { SHISEIDO Capcell core C18 column } \\
\text { SHISEIO Capcell coreC } 18 \text { column }\end{array}$ & $\begin{array}{l}\text { UFLC } \\
\text { UFLC }\end{array}$ & $\begin{array}{l}\text { Acetonitrile }- \text { water }(0.1 \% \text { formic acid }) \\
0.1 \% \text { formic acid in acetonitrile and water }\end{array}$ & $\begin{array}{l}\text { MS/MS } \\
\text { MS/MS }\end{array}$ & $\begin{array}{l}12 \\
12\end{array}$ & $\begin{array}{l}0.15 \mu \mathrm{gg} / \mathrm{kg} \\
0.05 \mu \mathrm{gg} / \mathrm{L}\end{array}$ & $\begin{array}{l}\text { Areca catechu } \\
\text { Yam }\end{array}$ & $\begin{array}{l}\mathrm{FB} 1, \mathrm{FB} 2 \\
\mathrm{FB} 1, \mathrm{FB} 2\end{array}$ & $\begin{array}{l}{[48]} \\
{[49]}\end{array}$ \\
\hline ACQUITY UPLC BEH C18 column & UHPLC & $\begin{array}{l}\text { Water containing } 0.1 \% \text { formic acid (ESI+) or } 0.1 \% \\
\text { ammonia (ESI) and acetonitrile }\end{array}$ & MS & 12 & $\begin{array}{l}0.32 \mu \mathrm{\mu g} / \mathrm{kg}, \\
0.08 \mathrm{~g} / \mathrm{kg}\end{array}$ & Radix Paeoniae Alba & $F B 1, F B 2$ & [50] \\
\hline ZIC-pHILIC (SeQuant) & LC & Aqueous ammonium formate & MS & 23 & $\begin{array}{l}0.3,1.3,1.3, \\
0.8,0.9,2.6\end{array}$ & $\begin{array}{l}\text { Apples, apricots, lettuce, } \\
\text { onion, wheat flour, } \\
\text { chickpeas }\end{array}$ & $\mathrm{FB} 1$ & [51] \\
\hline Poroshell 120 PFP column & UHPLC & $\begin{array}{l}\text { Ammonium formate and formic acid in Milli-Q water } \\
\text { and methanol (ESI+), and Milli-Q water and } \\
\text { acetonitrile (ESI-). }\end{array}$ & MS/MS & 17 & $1,1,3 \mu \mathrm{\mu g} / \mathrm{kg}$ & Pheretima & $\mathrm{FB} 1, \mathrm{FB} 2, \mathrm{FB} 3$ & [52] \\
\hline Kinetex $\mathrm{C} 18$ column & LC & Water-methanol with ammonium formate and formic & MS/MS & 33 & $1.7,3.9 \mu \mathrm{g} / \mathrm{L}$ & Maize & $F B 1, F B 2$ & [53] \\
\hline CORTECS C18 column & UPLC & Methanol-water with 0.5\% (v/v) formic acid & MS/MS & 30.3 & $15 \mu \mathrm{g} / \mathrm{kg}$ & Cereals (Wheat, corn, and & $\mathrm{FB} 1, \mathrm{FB} 2, \mathrm{FB} 3$ & [54] \\
\hline Acclaim $120 \mathrm{C} 18$ analytical column & HPLC & Acidified acetonitrile & Fluorescence & 30 & $30 \mu \mathrm{g} / \mathrm{kg}$ & $\begin{array}{l}\text { Corn based feed } \\
\text { rice }\end{array}$ & $\mathrm{FB}_{1}, \mathrm{FB}_{2}$ & [55] \\
\hline BEH C18 column & $\begin{array}{l}\text { LC-MS- } \\
\text { MS }\end{array}$ & Acidified water \& acetonitrile & MS-MS detection & $\begin{array}{l}2,4 \text { (only } \\
\text { hair) }\end{array}$ & $\begin{array}{l}2.5 \mathrm{~g} / \mathrm{L} /, \\
0.014,0.040, \\
0.12, \mathrm{ND} \mathrm{\mu g/L}\end{array}$ & $\begin{array}{l}\text { Pig plasma, urine, feces, } \\
\text { hair }\end{array}$ & $\mathrm{FB}_{1}$ & [56] \\
\hline $\begin{array}{l}\text { Nucleodur C18 Gravity SB column } \\
\text { Ascentis Express C18 }\end{array}$ & $\begin{array}{l}\mathrm{LC} \\
\mathrm{LC}\end{array}$ & $\begin{array}{l}\text { Acetonitrile }(2 \% \text { acetic acid)- - water }(0.1 \% \text { acetic acid) } \\
\text { Aqueous ammmonium formate }(0.1 \% \text { formic acid)- } \\
\text { aqueous methanol solution (ammonium formate, }+ \\
\text { formic acid, } 0.1 \% \text { ) }\end{array}$ & $\begin{array}{l}\text { MS } \\
\text { MS/MS }\end{array}$ & $\begin{array}{l}11.5 \\
30.1\end{array}$ & $\begin{array}{l}0.521 \mu \mathrm{gg} / \mathrm{L} \\
10.14,2.5 \\
0.625 \mu \mathrm{\mu g} / \mathrm{L}\end{array}$ & $\begin{array}{l}\text { Human blood } \\
\text { Milk }\end{array}$ & $\begin{array}{l}\mathrm{FB} 1 \\
\mathrm{FB} 1, \mathrm{FB} 2, \mathrm{FB} 3\end{array}$ & $\begin{array}{l}{[57]} \\
{[58]}\end{array}$ \\
\hline MNPs + Acquity UPLC@BEH C18 column & UPLC & $\begin{array}{l}\mathrm{MeOH} / \mathrm{H}_{2} \mathrm{O}(60: 40) \text { with ammonium acetate and } \\
\text { formic acid }\end{array}$ & MS/MS & 10 & $0.210 \mu \mathrm{g} / \mathrm{kg}$ & Vegetable oil & $\mathrm{FB} 1$ & [59] \\
\hline Kinetex XB-C18 100 Å column & HPLC & Methanol- water ( with ammonium formiate+ formic & MS/MS & 30 & $100 \mu \mathrm{g} / \mathrm{kg}$ & Cereal-derived products & $F B 1, F B 2$ & [60] \\
\hline Cortecs UHPLC C18 column & LC & $\begin{array}{c}\text { accid) } \\
\text { Water- } \\
\left.\mathrm{MeOH} \text { (with } \mathrm{NH}_{4} \mathrm{HCOO}+\mathrm{HCOOH}\right)\end{array}$ & MS/MS & 14.5 & $0.04 \mu \mathrm{g} / \mathrm{L}$ & Soy, oat and rice beverages & $\mathrm{FB} 1, \mathrm{FB} 2$ & [61] \\
\hline Gemini® C18 column & LC & Methano//water/acetic acid 10:89:1 (v/v/v) -97:2:1 & MS/MS & 20.5 & 3.2 (FB1), 2.4 & Maize-fufu & $\mathrm{FB} 1, \mathrm{FB} 2, \mathrm{FB} 3, \mathrm{FB} 4, \mathrm{FA} 1$ & [62] \\
\hline C18 column & UHPLC & $\begin{array}{l}\text { (with ammonium acetate) } \\
\text { Water- MeOH with formic acid and ammonium } \\
\text { formate }\end{array}$ & MS/MS & 11.25 & $\begin{array}{c}\mu \mathrm{g} / \mathrm{kg} \\
17.3,12.4,10.7, \\
9 \mu \mathrm{\mu g} / \mathrm{L}(\mathrm{FB} 1), \\
11.8,17.2,9,10 \\
\mu \mathrm{g} / \mathrm{L}(\mathrm{FB} 2)\end{array}$ & $\begin{array}{l}\text { Oat, soy,rice and bird seed } \\
\text { milk }\end{array}$ & $\mathrm{FB} 1, \mathrm{FB} 2$ & [63] \\
\hline Acquity BEH C18 column & UPLC & Water (ammonium acetate) - $\mathrm{MeOH}$ (formic acid) & MS/MS & 15 & $0.20,0.15 \mu \mathrm{g} / \mathrm{kg}$ & Alpinia oxyphylla & $\mathrm{FB} 1, \mathrm{FB} 2$ & [64] \\
\hline
\end{tabular}




\begin{tabular}{|c|c|c|c|c|c|c|c|c|}
\hline Eclipse Plus C8 RRHD column & $\begin{array}{l}\text { MA-D- } \mu- \\
\text { SPE with } \\
\text { UHPLC- } \\
\text { Q- }\end{array}$ & Water containing $0.1 \%$ formic acid-acetonitrile & MS & 9 & $\begin{array}{c}0.0068 \\
0.013 \\
0.0074 \\
0.0030 \mu \mathrm{g} / \mathrm{kg}\end{array}$ & $\begin{array}{l}\text { Peach seed, milk powder, } \\
\text { corn flour }\end{array}$ & FB1 & [65] \\
\hline $\begin{array}{l}\text { C18 column } \\
\text { Phenomenex Kinetex }\end{array}$ & $\begin{array}{l}\text { UPLC- } \\
\text { MS/MS }\end{array}$ & $\begin{array}{c}\text { Water } \\
\text { containing } 0.5 \mathrm{mM} \mathrm{NH} \text { Ac- } \mathrm{MeOH} \text { with } \\
0.1 \% \text { formic acid }\end{array}$ & MS/MS & 15 & $\begin{array}{l}0.25 \& 0.1 \\
(\mathrm{FB} 2) \quad \mu \mathrm{g} / \mathrm{kg}\end{array}$ & Lotus seed & $\mathrm{FB} 1, \mathrm{FB} 2$ & [66] \\
\hline $\begin{array}{l}\text { ZORBAX RRHD Eclipse Plus C18 } \\
\text { Gemini@ C18-column }\end{array}$ & $\underset{\text { LC }}{\text { UHPC }}$ & $\begin{array}{l}\text { 0.1\% formic acid solution - acetonitrile ( formic acid) } \\
\text { Methanol/water (with acetic acid and ammonium } \\
\text { acetate }\end{array}$ & $\begin{array}{l}\text { MS } \\
\text { MS/MS }\end{array}$ & 12 & $\begin{array}{l}1 \mu \mathrm{g} / \mathrm{h} \\
1 \mu \mathrm{gg} / \mathrm{kg}\end{array}$ & $\begin{array}{l}\text { Grape and wines } \\
\text { Dried date palm fruits }\end{array}$ & $\begin{array}{l}\text { FB1 } \\
\text { FB2 }\end{array}$ & {$[67]$} \\
\hline Acquity UPLC HSS T3 column & UPLC & (Formic acid \& ammonium formate) water-acetonitrile & MS/MS & 10 & $\begin{array}{l}0.15,0.09,0.04 \\
0.03,0.17 \mathrm{\mu g} / \mathrm{L}\end{array}$ & Broiler chicken plasma & $\begin{array}{l}\text { FB1, FB2, pHFB1a, } \\
\text { pHFB1b, HFB1 }\end{array}$ & [69] \\
\hline $\begin{array}{l}\text { Silica based particles bonded with C18-penta } \\
\text { fluorophenyl functions }\end{array}$ & LC-HRMS & Water- acetonitrile (both with formic acid) - $\mathrm{MeOH}$ & MS & 26 & $0.5 \mathrm{\mu g} / \mathrm{L}$ & Tea & FB1, FB2 & [70] \\
\hline Gemini-NX LC-column & LC & $\begin{array}{l}\text { Water - methanol acidified (both with ammonium } \\
\text { formate +formic acid) }\end{array}$ & MS/MS & 39 & $\begin{array}{c}1.5,0.3 \\
\text { (vegetables) } \\
\text { ug/kg }\end{array}$ & $\begin{array}{l}\text { Ready-to-eat food (cereals, } \\
\text { fish, legumes, vegetables, } \\
\text { meat) }\end{array}$ & FB1, FB2 & [71] \\
\hline Scherzo Sm-C18 column & HPLC & $\begin{array}{l}\text { Acetonitrile (ammonium acetate) - acetonitrile (formic } \\
\text { acid) }\end{array}$ & MS/MS & 26 & $2.4,2.3 \mu \mathrm{g} / \mathrm{kg}$ & Corn derived products & $\mathrm{FB} 1, \mathrm{FB} 2$ & [72] \\
\hline Acquity HSS T3 column & LC & Water-ACN (both acidified with $\mathrm{HAc}$ ) & MS/MS & 25 & $0.001 \mu \mathrm{g} / \mathrm{L}$ & Human urine & FB1 & [73] \\
\hline Waters ACQUITY HSS T3 column & UPLC & $\begin{array}{l}0.1 \% \text { formic acid and } 5 \mathrm{mM} \text { ammonium formate } \\
\text { (phase A) -methanol } \\
\text { (phase B). }\end{array}$ & MS/MS & 13 & $0.22 \mu \mathrm{g} / \mathrm{L}$ & Beer & $\mathrm{FB} 1, \mathrm{FB} 1$ & [74] \\
\hline Zorbax CX & UHPLC & Methano/ water $(1: 1 \mathrm{v} / \mathrm{v})$ with $0.1 \%$ acetic acid & MS/MS & $\begin{array}{l}3.6 \\
\text { (chromatogra } \\
\mathrm{m} \text { time) }\end{array}$ & $51.5,45.3 \mu \mathrm{g} / \mathrm{kg}$ & Rice & $\mathrm{FB} 1, \mathrm{FB} 2$ & [75] \\
\hline Kinetex Core-shell C18 & LC & $\begin{array}{l}\text { Water-methanol (both with ammonium formate and } \\
\text { formic acid ) }\end{array}$ & MS/MS & 25.5 & $8.3 \mu \mathrm{g} / \mathrm{kg}$ & Green coffee & FB1, FB2 & [76] \\
\hline Kinetex Biphenyl column & LC & $\begin{array}{l}0.01 \mathrm{Mammonium} \text { acetate }+0.1 \% \text { of acetic acid in } \\
\text { water/ MeOH - } 0.11 \mathrm{M} \text { ammonium acetate }+0.1 \% \text { of } \\
\text { acetic acid in water } \mathrm{MeOH}\end{array}$ & MS/MS & 16 & $\begin{array}{l}0.50, \\
1.56 \mathrm{\mu g} / \mathrm{kg}\end{array}$ & Animal feed & FB1, FB2 & [77] \\
\hline UPLC HSS T3 & LC & $\begin{array}{l}\text { Aqueous ammonium formate } 1 \mathrm{mM} \text { and formic acid } \\
1 \% \text { (phase A)-Ammonium formate } 1 \mathrm{mM} \text { and formic } \\
\text { acid } 1 \% \text { in methanol:water } 95: 3.9 \text { ) }\end{array}$ & MS/MS & 11 & $20 \mu \mathrm{g} / \mathrm{kg}$ & Nixtamalized Maize & $\mathrm{FB} 1, \mathrm{FB} 2$ & [185] \\
\hline Kinetex $2.6 \mu \mathrm{m} \mathrm{C18} \mathrm{100A}$ & UHPLC & $\begin{array}{l}\text { Aqueous acetic acid } 0.5 \% \text { (phase A)-Acetic acid 0.5\% } \\
\text { and isopropanol 99.5\% (phase B) }\end{array}$ & MS/MS & 11 & $0.03,0.01 \mu \mathrm{g} / \mathrm{L}$ & Kankankan & FB1, FB2 & [186] \\
\hline Gemini C18-column & LC-ESI & $\begin{array}{c}\text { Ammonium acetate } 5 \mathrm{mM} \text { with methanol/water/acetic } \\
\text { acid 10:89:1 (phase A) and 97:2:1 (phase B) }\end{array}$ & MS/MS & 18.5 & $\begin{array}{c}2.39,1.68,8.55 \\
\mathrm{\mu g} / \mathrm{kg}\end{array}$ & Dried Turkish figs & FB1, FB2, FB3 & [187] \\
\hline
\end{tabular}




\subsection{Immunosensors for detection of fumonisin}

The enzyme-linked immunosorbent assay (ELISA) for the determination of FB1 represents the foundation of different approaches. Competitive assays have been commonly employed for biosensing techniques, mostly because of the restriction produced by single epitopes on other types such as sandwich ELISA [85]. Some general procedures for a competitive immunoassay include a coating stage of antibody on the selected support, followed by the incubation with a mixture of free FB1 (sample) and functionalized toxin (horseradish peroxide (HRP)-FB1). After washing the unbound FB1 or HRP-FB1, different substrates can be added for the development of either a chemiluminescence or a colorimetric signal [86]. Some commercial kits are also based on a competitive scheme, in which capture antibodies, specific to a FB1 antibody, are coated on a well, where free FB1, enzyme-fumonisin and antibody are incubated. The bound HRP-fumonisin is then measured by incubating with a chromogen [42]. In some bulk experiments, magnetic nanobeads have been used as a support with a competitive binding role under the presence of FB1 and its biotinylated antibody [137].

Other modifications suggested the substitution of HRP with compounds such as glucose oxidase to produce hydrogen peroxide, an inducer of AuNP aggregation [133], and the application of genetically engineered antibodies [138]. A novel technique used a monoclonal antibody-rhodamine isothiocyanate (RBITC)-AuNPs probe for the competitive binding between OVA-FB1 and FB1, where cysteamine worked as a turn-on compound for revealing the degrees of fluorescence from the quenched probe [188].

This antigen-antibody interaction has been used, optimized and improved over the years; and commercially available ELISA kits and standardized ELISA protocols are still applied for method validation and comparison with novel biosensing developments [128, 130, 143, 166, 170]. As presented in Fig 4, electrochemical immunosensors have portrayed some of the lowest LODs $[101,109]$. For instance, the signal of an impedance sensor was modified by depositing quantum dotscarbon nanotubes on a glassy carbon electrode (GCE) for the immobilization of the corresponding antibody. In this case, the electron transfer resistance was enhanced after target binding, allowing LODs as low as $0.46 \mathrm{pg} / \mathrm{L}$ [101]. An electrochemical indirect competitive method was also refined by modifying a GCE with nanotubes-chitosan (undefined characteristics) and FB1-Bovine serum albumin (BSA). The remaining antibody after the incubation with free FB1 (sample) was able to bind FB1BSA, as well as an alkaline phosphate-labelled anti-antibody, whose substrate triggered the electrochemical signal with lower, yet good sensitivity of $2 \mathrm{ng} / \mathrm{L}$ [103]. The reduction of conductivity promoted by the antibody-antigen reaction was again explored for the immobilization of antibodies on nanotube-modified GCE, attaining a LOD of $3.8 \mathrm{pg} / \mathrm{L}$ [109]. In addition to electrochemical methods, surface-enhanced Raman scattering (SERS) competitive immunoassays were applied by combining FB1-BSA functionalized Au nanopillars with nanotags, consisting in AuNP simultaneously functionalized with anti-antibody and malachite green isothiocyanate (MGITC). The interaction between the primary antibody and high antigen concentrations resulted in a weak SERS signal, due to the absence of complex formation within free primary antibodies, nanopillars and nanotags, with a LOD of $0.00511 \mathrm{pg} / \mathrm{L}[126]$.

As noted in Table 3, immunosensors can be supported on different matrices, including optical fiber, well plates, glass slides, magnetic beads, magnetic nanoparticles, electrodes and chips. Yet another of the main advantages of using antibodies is the feasibility to be incorporated in paper-based biosensors. Paper matrices are presently relevant for the creation of portable, point-of-care, applicable and cheap devices [36]. The conjugation of antibodies with colloidal gold (gold nanoparticles) has been widely applied for the colorimetric detection of FB1 on nitrocellulose membranes $[88,91,94,95,98,99,102,107,113,116,120,136]$. Some modifications included the application of urchin-like and flower-like gold nanoparticles (AuNP), which slightly increased the sensitivity when compared to a spherical particle [107, 135]. 
Table 3. Immuno-based assays for the determination of FB1 ${ }^{1}$

\begin{tabular}{|c|c|c|c|c|c|c|c|c|}
\hline Support & Method & Labelling/Substrate & Measurement & $\begin{array}{c}\text { Assay } \\
\text { Time (min) }\end{array}$ & LOD & Sample & Fumonisin & Ref \\
\hline $\begin{array}{l}\text { 96-well immunoplates } \\
\text { ELISA kit } \\
\text { 96-well plate } \\
\text { Test tit } \\
\text { Optical fibre } \\
\text { Sample cell } \\
\text { Protein-A coated capillary column } \\
\text { Glass culture tube } \\
\text { Borosilicate glass slides } \\
\text { 96-well microplate }\end{array}$ & $\begin{array}{c}\text { ELISA } \\
\text { AgraQuant Total Fumonisin Assay Protocol } \\
\text { ELISA (RIDASCREEN } \text { (R) } \\
\text { ELISA } \\
\text { DC assay } \\
\text { SPR } \\
\text { Liposome-amplified competitive assay } \\
\text { Competition of unlabelled fumonisin } \\
\text { Competitive assay } \\
\text { ECL-ELISA }\end{array}$ & $\begin{array}{l}\text { HRP } \\
\text { Methanol-water } \\
\text { HRP } \\
\text { Antigen } \\
\text { FITC } \\
\text { Gold film } \\
\text { Liposome } \\
\text { Fluorescein } \\
\text { Biotin } \\
\text { HRP }\end{array}$ & $\begin{array}{l}\text { Optical density } \\
\text { Intensity of colour } \\
\text { Optical density } \\
\text { OD } \\
\text { Fluorescence } \\
\text { Reflected light intensity } \\
\text { Fluorescence } \\
\text { Fluorescence Polarization } \\
\text { Fluorescence } \\
\text { Fluorescence }\end{array}$ & $\begin{array}{l}150 \\
20 \\
55 \\
20 \\
24 \\
10 \\
<11 \\
2 \\
\sim 8 \\
60\end{array}$ & $\begin{array}{l}0.2 \mu \mathrm{\mu g} / \mathrm{L} \\
200 \mu \mathrm{gg} / \mathrm{kg} \\
25 \mathrm{\mu g} / \mathrm{g}, \\
200 \mu \mathrm{gg} / \mathrm{kg} \\
10 \mathrm{\mu g} / \mathrm{L} \\
50 \mu \mathrm{\mu g} / \mathrm{L} \\
1 \mathrm{\mu g} / \mathrm{L} \\
500 \mathrm{\mu g} / \mathrm{kg} \\
250 \mathrm{\mu g} / \mathrm{L} \\
0.09 \mu \mathrm{\mu g} / \mathrm{L}\end{array}$ & $\begin{array}{c}\text { Corn } \\
\text { Corn } \\
\text { Tunisian foods and feed } \\
\text { Maize } \\
\text { Corn } \\
\text { PBS } \\
\text { TBS } \\
\text { Maize } \\
\text { PBSTB } \\
\text { Cereals }\end{array}$ & $\begin{array}{c}\mathrm{FB1} \\
\mathrm{FBS} \\
\mathrm{FBB}+\mathrm{FB} 2 \\
\mathrm{FB1} 1 \mathrm{FB} 2 \\
\mathrm{FB} 1 \\
\mathrm{FB1} \\
\mathrm{FB} 1 \\
\mathrm{FB1} \\
\mathrm{FB} 1 \\
\mathrm{FB1}\end{array}$ & $\begin{array}{l}{[38]} \\
{[41]} \\
{[42]} \\
{[45]} \\
{[81]} \\
{[82]} \\
{[83]} \\
{[84]} \\
{[85]} \\
{[86]}\end{array}$ \\
\hline $\begin{array}{l}\text { DMA-NAS-MAPS treated glass } \\
\text { NC membrane } \\
\text { Luminex } 100 \text { microspheres } \\
\text { SPGE } \\
\text { NC membrane } \\
\text { Aldehydelized } \\
\text { glass slides }\end{array}$ & $\begin{array}{l}\text { Competitive immunoassay } \\
\text { LFIA } \\
\text { Indirect competitive fluid array } \\
\text { DC assay } \\
\text { LFIA } \\
\text { Specific competitive reactions }\end{array}$ & $\begin{array}{l}\text { Streptavidin-AP/NBT/BCIP } \\
\text { Colloidal Gold } \\
\text { Biotin } \\
\text { HRP-TMB } \\
\text { Colloidal Gold } \\
\text { Ag conjugates }\end{array}$ & $\begin{array}{l}\text { Colorimetric } \\
\text { Line intensity } \\
\text { Fluorescence cytometry } \\
\text { Chronoamperometry } \\
\text { Line intensity } \\
\text { Fluorescence }\end{array}$ & $\begin{array}{l}65 \\
4 \\
60 \\
45 \\
10 \\
90\end{array}$ & $\begin{array}{c}43 \mu \mathrm{\mu g} / \mathrm{L} \\
199 \mathrm{\mu g} / \mathrm{kg} \\
0.3 \mathrm{Hg} / \mathrm{L} \\
5 \mathrm{\mu g} / \mathrm{L} \\
120 \mathrm{\mu g} / \mathrm{L} \\
109.06 \mathrm{\mu g} / \mathrm{L}\end{array}$ & $\begin{array}{l}\text { Binding buffer } \\
\text { Maize } \\
\text { Grain Products } \\
\text { Corn } \\
\text { Maize } \\
\text { Wheat }\end{array}$ & $\begin{array}{l}\mathrm{FB} 1 \\
\mathrm{FB} 1 \\
\mathrm{FB} 1 \\
\mathrm{FB1}, \mathrm{FB} 2 \\
\mathrm{FB1} \\
\mathrm{FB} 1\end{array}$ & $\begin{array}{l}{[87]} \\
{[88]} \\
{[89]} \\
{[90]} \\
{[91]} \\
{[92]}\end{array}$ \\
\hline $\begin{array}{l}\text { NC strip } \\
\text { NC membrane strip } \\
\text { NC membrane } \\
96 \text {-well microplate } \\
\text { Paramagnetic beads }\end{array}$ & $\begin{array}{l}\text { Competitive lateral flow immunoassay } \\
\text { One-step competitive Immunochromatographic } \\
\text { LFIA } \\
\text { IC ELISA } \\
\text { Inhibition immunoassay }\end{array}$ & $\begin{array}{c}\text { HRP } \\
\text { AuNP } \\
\text { Protein A-gold } \\
\text { HRP } \\
\text { Mycotoxin-R-Phycoerythrin }\end{array}$ & $\begin{array}{c}\mathrm{CL} \\
\text { Colour density } \\
\text { Line intensity } \\
\text { Absorbance } \\
\text { Dose-response cytometry } \\
\text { (Fluorescence) }\end{array}$ & $\begin{array}{l}15 \\
10 \\
30 \\
70 \\
50\end{array}$ & $\begin{array}{c}2.5 \mathrm{\mu g} / \mathrm{L} \\
2.5 \mathrm{gg} / \mathrm{L} \\
3200 \mathrm{~g} / \mathrm{kg} \\
8.32 \mathrm{gg} / \mathrm{kg} \\
170,1270 \\
\mu \mathrm{g} / \mathrm{kg}\end{array}$ & $\begin{array}{l}\text { Maize } \\
\text { Maize } \\
\text { Maize } \\
\text { Corn } \\
\text { Maize, wheat }\end{array}$ & $\begin{array}{c}\mathrm{FB} 1, \mathrm{FB} 2 \\
\mathrm{FB} 1+\mathrm{FB} 2+\mathrm{FB} 3 \\
\mathrm{FB} 1 \\
\mathrm{FB} 1 \\
\mathrm{FB} 1+\mathrm{FB} 2\end{array}$ & $\begin{array}{l}{[93]} \\
{[94]} \\
{[95]} \\
{[96]} \\
{[97]}\end{array}$ \\
\hline $\begin{array}{l}\text { NC membrane } \\
\text { NC membrane } \\
\text { PrG functionalized magnetic beads } \\
\text { SPCEs }\end{array}$ & $\begin{array}{c}\text { LFDIA } \\
\text { Immunochromatographic strip } \\
\text { DC multi-channel electrochemical immunoassay }\end{array}$ & $\begin{array}{l}\text { Colloidal Gold } \\
\text { Colloidal gold } \\
\text { HRP }\end{array}$ & $\begin{array}{l}\text { (Fuorescence) } \\
\text { Line intensity } \\
\text { Visual detection } \\
\text { Current }\end{array}$ & $\begin{array}{c}30 \\
3 \\
40\end{array}$ & $\begin{array}{l}\mu \mathrm{\mu g} / \mathrm{kg} / \mathrm{L} \\
5.2 \mu \mathrm{gg} / \mathrm{L} \\
0.58 \mu \mathrm{\mu g} / \mathrm{L}\end{array}$ & $\begin{array}{l}\text { Corn } \\
\text { Cereal } \\
\text { Cereals }\end{array}$ & $\begin{array}{l}\mathrm{FB} 1 \\
\mathrm{FB1} \\
\mathrm{FB} 1\end{array}$ & $\begin{array}{r}{[98]} \\
{[99]} \\
{[100]}\end{array}$ \\
\hline GCE/PT & Impedimetric immunosensor & PDMA-MWCNT & EIS & - & $\begin{array}{c}0.00000046 \\
\mu \mathrm{gg} / \mathrm{L} \\
14 \mathrm{\mu g} / \mathrm{kg}\end{array}$ & $\begin{array}{l}\text { Methanol } \\
\text { Corn }\end{array}$ & $\begin{array}{l}\mathrm{FB} 1 \\
\mathrm{FB} 1\end{array}$ & [101] \\
\hline $\begin{array}{l}\text { NC Strip } \\
\text { SWNTs//CS electrode } \\
\text { SPCEs-Magnetic beads } \\
\text { 96-well microplate } \\
\text { Microplate reader }\end{array}$ & $\begin{array}{l}\text { LFIA } \\
\text { Indirect competitive binding } \\
\text { Competitive multi-immunoassay } \\
\text { Biopanning } \\
\text { FPIA }\end{array}$ & $\begin{array}{c}\text { HRP } \\
\text { Alkaline phosphatase } \\
\text { HRP } \\
\text { Ab2B Nb/HRP } \\
\text { FITC }\end{array}$ & $\begin{array}{c}\mathrm{CL} \\
\text { Electrochemical } \\
\text { Amperometric } \\
\mathrm{OD} \\
\text { Fluorescence Polarization }\end{array}$ & $\begin{array}{c}30 \\
180.11 \\
60 \\
\sim 60 \\
<30\end{array}$ & $\begin{array}{c}14 \mu \mathrm{\mu g} / \mathrm{kg} \\
6 \mathrm{\mu g} \\
6 \mathrm{\mu g} / \mathrm{kg} \\
0.002 \mu \mathrm{\mu g} / \mathrm{L} \\
0.33 \mu \mathrm{\mu g} / \mathrm{L} \\
0.15 \mu \mathrm{h} \\
157.4 \\
290.6 \mathrm{ug} / \mathrm{kg}\end{array}$ & $\begin{array}{l}\text { Corn } \\
\text { Maize } \\
\text { Corn } \\
\text { CRM, beer } \\
\text { PBS } \\
\text { Maize }\end{array}$ & $\begin{array}{l}\text { FB2+FB3 } \\
\text { FB1 } \\
\text { FB1 } \\
\text { FB1,FB2,FB3 } \\
\text { FB1, FB2 } \\
\text { FB1, } \\
\text { FB2. }\end{array}$ & $\begin{array}{l}{[102]} \\
{[103]} \\
{[104]} \\
{[105]} \\
{[106]}\end{array}$ \\
\hline $\begin{array}{l}\text { NC membrane } \\
\text { NC membrane } \\
\text { PPy/ErGO SPE } \\
\text { GCE }\end{array}$ & $\begin{array}{l}\text { Competitive small molecule detection } \\
\text { Competitive small molecule detection } \\
\text { Label-free electrochemical immunosensing } \\
\text { Electrochemical impedance spectroscopy }\end{array}$ & $\begin{array}{c}\text { UGNS } \\
\text { AuNP } \\
\text { AuNP } \\
\text { PDMA-MWCNT }\end{array}$ & $\begin{array}{c}\text { Colour intensity } \\
\text { Colour intensity } \\
\text { Current } \\
\text { Electron transfer resistance }\end{array}$ & $\begin{array}{l}<5 \\
<5 \\
40\end{array}$ & $\begin{array}{c}290.6 \mu \mathrm{gg} / \mathrm{kg} \\
5 \mu \mathrm{hg} \\
20 \mu \mathrm{Lg} / \mathrm{L} \\
4.2 \mu \mathrm{\mu g} / \mathrm{kg} \\
0.0000038 \\
\mu \mathrm{gg} / \mathrm{L}\end{array}$ & $\begin{array}{l}\text { Grains } \\
\text { Grains } \\
\text { Corn } \\
\text { Corn }\end{array}$ & $\begin{array}{l}\mathrm{FB2} \\
\mathrm{FB1} \\
\mathrm{FB1} \\
\mathrm{FB1} \\
\mathrm{FB1}\end{array}$ & $\left.\begin{array}{l}{[107]} \\
{[107]} \\
{[108]} \\
{[109]}\end{array}\right]$ \\
\hline $\begin{array}{l}\text { NC membrane } \\
\text { Hi-Flow Plus membranes } \\
\text { Microbead }\end{array}$ & $\begin{array}{l}\text { Immunochromatographic strip test } \\
\text { Competitive reaction } \\
\text { Flow immunocytometry }\end{array}$ & $\begin{array}{l}\text { DR-AuNP } \\
\text { AunP } \\
\text { Phycoerythrin }\end{array}$ & $\begin{array}{l}\text { Visual detection } \\
\text { Coloration } \\
\text { Fluorescence }\end{array}$ & $\begin{array}{l}10 \\
15 \\
45\end{array}$ & $\begin{array}{l}1000 \mathrm{\mu g} / \mathrm{kg} \\
0.6 \mathrm{\mu g} / \mathrm{Lg} \\
116 \mu \mathrm{kg}\end{array}$ & $\begin{array}{l}\text { Maize flour } \\
\text { Maize } \\
\text { Maize }\end{array}$ & $\begin{array}{l}\mathrm{FB} 1 \\
\mathrm{FB1} \\
\mathrm{FB1}\end{array}$ & $\begin{array}{l}{[110]} \\
{[111]} \\
{[112]}\end{array}$ \\
\hline NC strips & Competitive assay & Colloidal gold & Colour intensity & 10 & $0.24 \mu \mathrm{g} / \mathrm{L}$ & Agricultural products & FB1 & [113] \\
\hline $\begin{array}{l}\text { Plates } \\
\text { Mimotope on ARChip Epoxy slides } \\
\text { NC high-flow plus membranes }\end{array}$ & $\begin{array}{l}\text { IC ELISA } \\
\text { Competitive binding inhibition } \\
\text { Competitive binding inhibition }\end{array}$ & $\begin{array}{l}\text { IgG-HRP } \\
\text { Alexa Fluor } 647-\text { lgG } \\
\text { AuNP/ HRP-labelled lgG }\end{array}$ & $\begin{array}{l}\text { Absorbance } \\
\text { Fluorescence } \\
\text { Colour }\end{array}$ & $\begin{array}{l}68 \\
210 \\
10\end{array}$ & $\begin{array}{l}0.08 \mu \mathrm{g} / \mathrm{L} \\
11.1 \mu \mathrm{\mu} / \mathrm{L} \\
25 \mu \mathrm{g} / \mathrm{L}\end{array}$ & $\begin{array}{l}\text { Agricultural products } \\
\text { Maize, wheat } \\
\text { Corn }\end{array}$ & $\begin{array}{l}\mathrm{FB} 1 \\
\mathrm{FB1} \\
\mathrm{FB} 1\end{array}$ & $\begin{array}{l}{[113]} \\
{[114]} \\
{[115]}\end{array}$ \\
\hline $\begin{array}{l}\text { Nitrocellulose membrane } \\
\text { 96-well microplates } \\
\text { Mycotoxin-protein conjugates on chip } \\
\text { (MZI) }\end{array}$ & $\begin{array}{c}\text { LFIA } \\
\text { Competitive assay } \\
\text { Primary ( (yycotoxin//protein } \\
\text { conjugates - anti-mycotoxin specific mAbs) and } \\
\text { secondary } \\
\text { immunoreaction (immune adsorbed mAbs- IgG } \\
\text { antibody) }\end{array}$ & $\begin{array}{c}\text { AuNP/ CdSe/ZnS QD } \\
\text { AuNF@FeTPPCl + TMB } \\
\text { Label-free }\end{array}$ & $\begin{array}{l}\text { Fluorescence } \\
\text { Colour } \\
\text { Phase shift }\end{array}$ & $\begin{array}{l}15 \\
40 \\
12\end{array}$ & $\begin{array}{c}62.5 \mu \mathrm{\mu g} / \mathrm{kg} \\
0.05 \mu \mathrm{\mu g} / \mathrm{L} \\
5.6 \mu \mathrm{g} / \mathrm{L}\end{array}$ & $\begin{array}{l}\text { Maize flour } \\
\text { Buffer } \\
\text { Beer }\end{array}$ & $\begin{array}{l}\mathrm{FB1}, \mathrm{FB} 2 \\
\mathrm{FB} 1 \\
\mathrm{FB} 1\end{array}$ & $\left.\begin{array}{l}{[116]} \\
{[117]} \\
{[118]}\end{array}\right]$ \\
\hline
\end{tabular}




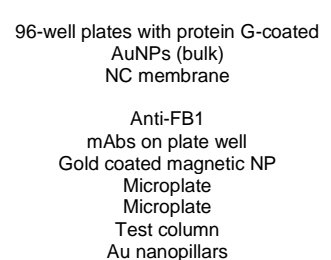

$$
\begin{gathered}
\text { Anti-FB1 } \\
\text { mAbs on plate we }
\end{gathered}
$$$$
\begin{aligned}
& \text { mAbs on plate well } \\
& \text { Gold coated magnetic NP }
\end{aligned}
$$$$
\begin{aligned}
& \text { Microplate } \\
& \text { Microplate }
\end{aligned}
$$$$
\begin{aligned}
& \text { Test column } \\
& \text { Aunanopillars }
\end{aligned}
$$$$
\text { NC membrane }
$$

Anti- FB1 mAb in microtiter wells

ITO coated glass integrated with PDMS

Superparamagnentic carbooxylated
XMAP@ microspheres

NC membranes
GONC on DEP electrod

96 well plates with protein-G and BSA

$$
\begin{aligned}
& \text { NC membrane } \\
& \text { NC membrane } \\
& \text { Nanomagnetic beads } \\
& \text { NC membrane } \\
& \text { NC membrane }
\end{aligned}
$$

\section{Microplate-OVA-FB1}

NC membrane
NC-membrane
Competitive
immunoassay

immunoassay
Competitive inhibition reaction

Competitive fluorescence ELISA

Competitive CLIA

DC-DELISA
IATC

Surface-enhanced Raman scattering

Direct competition

Non-competitive idiometric nanobodies phage

Three-electrode electrochemical sensor

Quadplex FCIA

Multiplex ICr assay
Electroactivity reduction with biorecognition

Competitive Plasmonic ELIIIA
Competitive multiplex ICr Assay

ICr strip

Multiplex ICr tes

Competitive solid-phase assay

Competitive ICr strip
Smartphone-based multiplex LFIA

\section{Competitive immunoreaction}

Competitive ICr strip

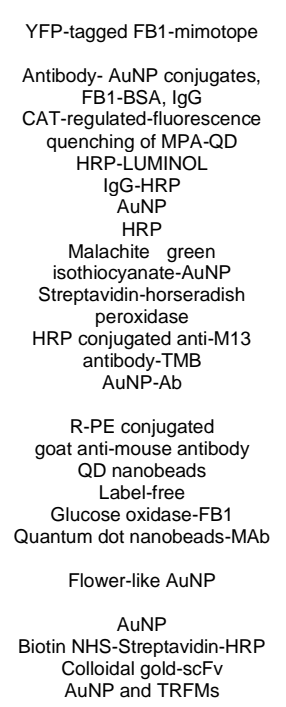

Cysteamine on mAb-RBITC-
AuNPs

AuNPs
QDNBs-mAb
Eu-FM-mAB

\section{Fluorescence \\ Visual detection \\ Fluorescence \\ Fluorescence \\ Absorbance
Absorbance
Color intensity \\ Raman intensity}

Enhanced chemiluminescence

Absorbance

Current

Fluorescence
CV/DPV

Absorbance
Fluorescence (test line/ control

line)
Color intensity

Colour intensity
OD

Color Intensity
Ratio T/C line color \&
fluorescence

Fluorescence

Fluorescence
Time-resolved fluorescence

\section{5}

10

$1.1 \mu \mathrm{g} / \mathrm{L}$

$30 \mu \mathrm{g} / \mathrm{L}$

$0.33 \mu \mathrm{g} / \mathrm{L}$

$0.027 \mathrm{mg} / \mathrm{L}$

$0.078 \mathrm{gg} / \mathrm{L}$
$12.5 \mu \mathrm{L}$
$20 \mu \mathrm{\mu g} / \mathrm{kg}$
0.09511

$\mathrm{pg} / \mathrm{mL}$
$0.24 \mu \mathrm{g} / \mathrm{L}$

$0.19 \mu \mathrm{g} / \mathrm{L}$

$0.097 \mu \mathrm{g} / \mathrm{L}$

$2.45 \mu \mathrm{g} / \mathrm{L}$

$20 \mu \mathrm{g} / \mathrm{L}$

$294 \mu \mathrm{\mu g} / \mathrm{L}$
$0.31 \mu g / \mathrm{L}$
$1.58 \mu \mathrm{h} / \mathrm{L}$

$5 \mu \mathrm{g} / \mathrm{L}$

$60 \mu \mathrm{g} / \mathrm{L}$

$0.21 \mu \mathrm{\mu g} / \mathrm{L}$
$2.5 \mathrm{~g} / \mathrm{L}$
$0.59 \mathrm{\mu g} / \mathrm{kg}$

(C)

$0.42 \mu \mathrm{\mu g} / \mathrm{kg}$
$(\mathrm{F})$

$0.023 \mu \mathrm{g} / \mathrm{L}$

$60 \mu \mathrm{\mu g} / \mathrm{L}$
$8.26 \mu \mathrm{kg}$
Wheat

Corn

Corn

Cereals

Corn
Corn
Maize

Corn samples

Corn

Corn

Milk

Maize
PBS-T
Maize

Cereals

Chinese traditional

medicine
Wheat and corn

Maize

Maize, wheat, bran

Maize

Wheat. corn
Corn flour, whea

Corn, corn flour, wheat,
rice, brown rice
FB1, FB2 [119]

FB1 [120]

FB1

$\mathrm{FB} 1$
$\mathrm{FB} 1, \mathrm{FB} 2, \mathrm{FB} 3$

$\begin{array}{cc}\mathrm{FB} 1 & {[124} \\ \mathrm{FB} 1, \mathrm{FB} 2, \mathrm{FB} 3 & {[125} \\ \mathrm{FB} & {[120}\end{array}$

FB1 [127]

FB1 [128]

FB1 [129]

FB1 [130]

$\begin{array}{ll}\text { FB1 } & {[131]} \\ \text { FB1 } & {[132]} \\ \text { FB1 } & {[133]} \\ \text { FB1 } & {[134]}\end{array}$

FB1 [135]

$\begin{array}{ll}\text { FB1 } & {[136]} \\ \text { FB1 } & {[137]} \\ \text { FB1 } & {[138]} \\ \text { FB1 } & {[139]}\end{array}$

FB1, FB2, FB3

$\begin{array}{ll}\text { FB1 } & {[189} \\ \text { FB1 } & {[190}\end{array}$

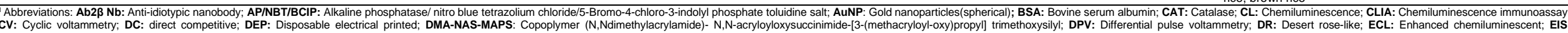

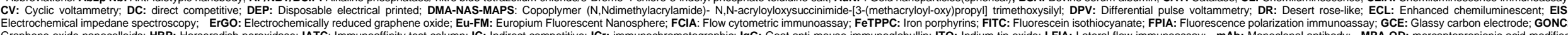

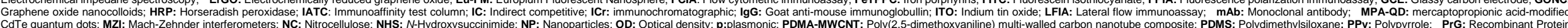

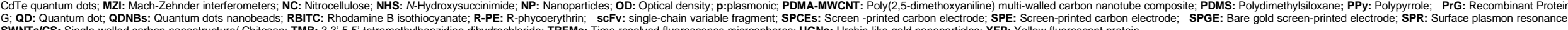


As an alternative to color intensity measurements, a chemiluminescent substrate could be incubated with HRP for a slight improvement of the LOD [93, 127], or the application of quantum dot (QD) in which a radiometric analysis revealed a constant signal from the test line with biotin-BSA, compared to the calibration with anti-mouse IgG [134]. Nevertheless, the application of fluorescent QDs not always result in an improved sensitivity, as reported for a nitrocellulose strip for the detection of FB1 (LOD: $60 \mu \mathrm{g} / \mathrm{L}$ ) , ZEN and OTA with a monoclonal antibody-QD probe placed on the conjugate pad, through the competitive interaction with mycotoxin-BSA at the test line [189], and a mABEuropium fluorescent nanoparticle with FB1 (LOD: $8.26 \mu \mathrm{g} / \mathrm{L}$ ) and FB1-BSA (Test line) [190]. An advantage of paper-based biosensors is the possibility of performing smartphone-based analysis, as already achieved on colorimetric and fluorescent signals [139]. Notwithstanding the multiple modifications, most of the differences among paper-based and other types of immunosensors can be explained in terms of the different antibodies selected and employed in each method.

\subsection{Other methods}

Alternatives to the extensively known immunologic and chromatographic techniques include chemometric, electrochemical and colorimetric analysis, as shown in Table 4. In SERS, the spectral variations of extracted samples mixed with $\mathrm{Ag}$ dendrites were measured on a quartz plate [140], while innovative, promising and more robust techniques incorporated the use of molecularly imprinted polymer nanoparticles (MIPs). Commonly polymerized with monomers such as methacrylic acid (MAA), ethylene glycol methacrylate (EGMP), N-isopropylacrylamide (NIPAM), N,N'-methylene-bisacrylamide (BIS), N-tert-butylacrylamide (TBAm), and N-(3-Aminopropyl) methacrylamide hydrochloride (NAPMA); MIPs have functioned as a replacement of primary antibodies; in which the utilization of FB1 as template molecule enhanced the performance, selectivity, thermal stability, and easy manufacturing of this technique. Once the MIPs are synthesized, the general procedure is similar to ELISA, where free FB1 competes with a FB1-HRP conjugate, where the latter reacts with a substrate (TMB: 3,3',5,5'-tetramethylbenzidine), bearing a colorimetric response. Such mechanism reduces the limit of the detection to $4.4 \mathrm{ng} / \mathrm{L}$ [141] and $1.37 \mathrm{ng} / \mathrm{L}$ [143], while an improvement on the silanisation step yielded more MIPSs and allowed the quantification of FB in maize, with a lower LOD equivalent to $1 \mathrm{ng} / \mathrm{L}$ [145]. Recent alternative methods suggested the chemical modification of FB1 prior to its quantification assay, where alkaline hydrolysis with $\mathrm{KOH}$ was proposed to reduce steric hindrance, allowing the formation of hydrogen bonds between hydrolysed fumonisin (HFB1) and the $\mathrm{NH}_{2}$ groups in cysteamine functionalized AuNP [146]. Likewise, a derivatization step between FB1 and a fluorescent derivative was necessary for spectra acquisition on a nylon membrane [146]. Besides, as already observed for some immunoassays, electrochemical methods were combined with MIPs, for a reduction on the limit of detection. A GCE modified with AuNPs and Ru@SiO 2 in chitosan (undefined characteristics), was proved as favorable support to produce MIPs generating electrochemiluminescent estimations with a LOD of $0.35 \mathrm{ng} / \mathrm{L}$ [142]. In a similar approach, an iridium tin oxide (ITO) electrode modified with CdS quantum dots, chitosan (undefined characteristics) and graphene oxide worked as the UV polymerization area, in which the resulting MIPs were used for photoelectrochemical evaluation of FB1 levels as low as $4.7 \mathrm{ng} / \mathrm{L}$ [144]. The application of nanoMIPs in electrochemical measurements (EIS, DPV) allowed the achievement of LODs as low as $21.6 \mathrm{fg} / \mathrm{L}$, which so far is the lowest value reported for FB1 [191].

On the other hand, capillary electrophoresis (CE) was initially reported in 1995 as a different technique with greater capability for the separation of FB1 to that from LC, where either its integration with MS detection or the quantification of fluorescent derivatives were utilized in the analysis of corn [192, 193]. Subsequent CE approaches explored the performance of fluorescein isothiocyanate for the derivatization of FB1 [194], and its application in the competitive binding of $m A b$ by labeled (derivatized) and unlabeled FB1, for the CE of the remaining fluorescein-FB1 [195]. Despite the advantages of CE in terms of the column efficiency, speed, reduction of organic solvents [193], the high limit of detections restricted any further applications. After two decades only one recent work on the application of coated $\left(C_{1}\right)$ and uncoated capillaries resulted in a relatively high LOD of $156 \mu \mathrm{g} / \mathrm{L}$ for the analysis of rice and fusarium microconidia by CE-MS [196], which denotes an opportunity for exploring, refining and optimizing more CE options for the determination of FB1 and other analogues. 
Table 4. Other methods for FB1 determination ${ }^{1}$

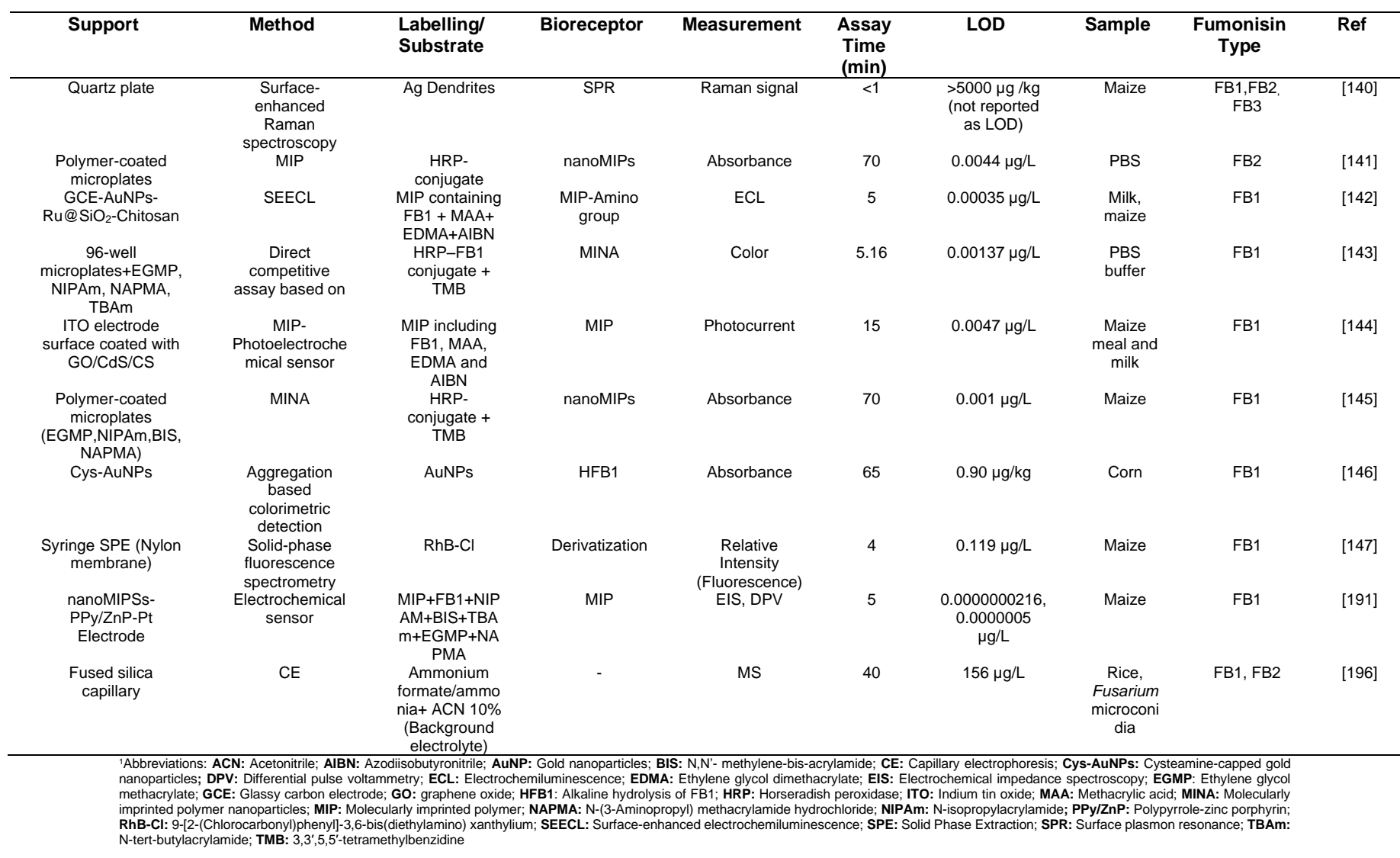

\section{Aptamer-based determination of FB1}

Aptamers are single-stranded DNA or RNA with high molecular recognition towards different types of molecules. Such probes exhibit diverse binding affinities and target selectivity and can discriminate even slight chiral differences. Aptamers are selected by a technique called Systematic Evolution of Ligands by Exponential enrichment (SELEX) in which a DNA library is incubated with the target or other relevant molecules, followed by the amplification of potential binders after several selection and discrimination rounds [148]. So far, two aptamers composed by 96 and 80 nucleotides, have been reported through SELEX and utilized in different biosensing approaches [148, 149], all the aptamer-based sensors are chronologically described in Table 5, while the binding and functionalization conditions are illustrated in Table 6. From the 31 aptasensors found in the literature, 24 utilized the $96 \mathrm{nt}$ aptamer [148], one method applied a shortened version (60 nt) from this first sequence [159], one platform included the second $80 \mathrm{nt}$ aptamer[156], two biosensors manipulated a condensed version (40 nt) of the second main aptamer [164, 167], and three references did not specify their single-stranded (ss) DNA sequence $[175,177,198]$. The schematic representation of each type of aptasensor assay is illustrated in Fig 6a a for the biosensors involving the initial $96 \mathrm{nt}$ aptamer, and in Fig $6 \mathrm{~b}$ for the application of the subsequent and not specified aptamers. It should be noted that the most recent sequences have not replaced the first reported aptamer, and current biosensing designs still apply the $96 \mathrm{nt}$ ssDNA molecule with high sensitivity and specificity. 
Table 5. Aptasensors for the determination of FB1 ${ }^{1}$

\begin{tabular}{|c|c|c|c|c|c|c|c|c|c|}
\hline Support & Labelling & Measurement & $\begin{array}{l}\text { Detectio } \\
\text { n Time } \\
(\min )\end{array}$ & $\begin{array}{l}\text { Extraction } \\
\text { Time } \\
\text { (min) }\end{array}$ & $\begin{array}{c}\text { Sample } \\
\text { Preparatio } \\
\text { n Steps }\end{array}$ & $\begin{array}{l}\mathrm{LOD} \\
\mu \mathrm{g} / \mathrm{L}\end{array}$ & Sample & Specificity Test $^{2}$ & Ref \\
\hline GO & $\begin{array}{l}\text { UCNPs with Er } \\
\text { and Tm }\end{array}$ & $\begin{array}{l}\text { Fluorescence } \\
\text { spectra }\end{array}$ & 200 & - & - & 0.1 & PBS & $\begin{array}{l}\text { OTA, AFB1, AFB2, } \\
\text { AFG1, AFG2, FB2, } \\
\text { ZEN }\end{array}$ & [151] \\
\hline $\begin{array}{l}\text { Carboxilated } \\
\text { MNPs/MB }\end{array}$ & UCNPs & Fluorescence & 100 & $>2$ & 7 & 0.01 & Maize & - & [152] \\
\hline Centrifuge tubes & AuNP-cDNA & Absorbance & 35 & 30 & 3 & 0.125 & Beer & & [153] \\
\hline SPCMs & $\begin{array}{l}\text { FITC- } \\
\text { Complementary } \\
\text { DNA }\end{array}$ & Fluorescence & 60 & 135 & 3 & 0.00016 & Cereal & AFB1, OTA, FB2 & [154] \\
\hline $\begin{array}{l}\text { cDNA modified Au } \\
\text { electrode }\end{array}$ & Au NPs-Ir & ECL & 120.41 & - & 5 & 0.27 & Wheat flour & $\begin{array}{l}\text { OTA, AFT, L-cystein, } \\
\text { BSA }\end{array}$ & [155] \\
\hline $\begin{array}{l}\text { GCE-AuNPs } \\
\text { Au coated silicon }\end{array}$ & $\begin{array}{l}\text { Label free } \\
\text { Label Free }\end{array}$ & $\begin{array}{l}\text { EIS } \\
\text { Deflection }\end{array}$ & $\begin{array}{l}30 \\
30\end{array}$ & 745 & 8 & 0.0014 & $\begin{array}{l}\text { Maize } \\
\text { Buffer }\end{array}$ & AFB1, ZEN, T-2 toxin & {$[156]$} \\
\hline cantilever beams & & & & & & & & & \\
\hline $\begin{array}{l}\text { GCE-AuNPs-capture } \\
\text { DNA }\end{array}$ & GS-TH & $\mathrm{CV}$ & 25.11 & - & - & 0.001 & $\begin{array}{l}\text { Ultra-pure } \\
\text { water }\end{array}$ & $\begin{array}{l}\text { AFB1, OTA, ZEN, } \\
\text { DON }\end{array}$ & [158] \\
\hline $\begin{array}{l}\text { cDNA } \\
\text { (Corning }{ }^{\circledR} \text { Costar }{ }^{8} 9 \\
6 \text {-Well Cell Culture } \\
\text { Plates) }\end{array}$ & PicoGreen & $\begin{array}{l}\text { Fluorescence } \\
\text { intensity }\end{array}$ & 25 & - & 2 & 0.1 & Milk & $\begin{array}{l}\text { CTN,OTA, AFB1, } \\
\text { ZEN }\end{array}$ & [159] \\
\hline $\begin{array}{l}\text { SPCE-PDMS } \\
\text { microcell }\end{array}$ & AuNPs & $\begin{array}{l}\text { Impedance } \\
\text { signal }\end{array}$ & 30 & 735 & 7 & 0.0034 & Corn & FB2, OTA, AFB1 & [160] \\
\hline SPCM & $\begin{array}{l}\text { cy3 modified } \\
\text { aptamer }\end{array}$ & Fluorescence & 90 & 751 & 7 & 0.01104 & Cereals & AFB1, OTA & [161] \\
\hline $\begin{array}{l}\mathrm{SiO}_{2} \text { spheres/ } \\
\mathrm{Fe}_{3} \mathrm{O}_{4} @ \mathrm{Au} \text { Magnetic } \\
\text { Beads-cDNA }\end{array}$ & $\mathrm{PbS}$ QD & SWV (current) & 65 & 15 & 4 & 0.02 & Maize & ОТА, OTB, AFB1 & [162] \\
\hline $\begin{array}{l}\text { Reduce graphene/Ni/ } \\
\text { Pt NPs micromotors }\end{array}$ & $\begin{array}{l}\text { Fluorescein } \\
\text { amidine (FAM) } \\
\text { labelled aptamer }\end{array}$ & $\begin{array}{l}\text { Fluorescence } \\
\text { intensity }\end{array}$ & 15 & $\begin{array}{l}\text { Maize: } 30 \\
\text { Beer: } 20 \\
\text { Whine: - }\end{array}$ & $4,1,1$ & 0.4 & Maize, Beer & OTA & [163] \\
\hline $\begin{array}{l}\text { Graphene modified } \\
\text { GCE }\end{array}$ & Label free & $\begin{array}{l}\text { Impedimetric } \\
\text { signal }\end{array}$ & 30 & - & - & 0.0123 & Tris buffer & - & [164] \\
\hline Centrifuge tube & $\begin{array}{l}\text { FITC- } \\
\text { Complementary } \\
\text { DNA }\end{array}$ & Fluorescence & 21 & - & - & 7.21 & Buffer & $\begin{array}{l}\text { AFB1, AFB2, OTA, } \\
\text { FB2 (response) }\end{array}$ & [165] \\
\hline $\begin{array}{l}\mathrm{TiO}_{2} \text { modified porous } \\
\text { silicon }\end{array}$ & $\begin{array}{l}\text { Cy3 labelled } \\
\text { aptamer-BHQ2 } \\
\text { labelled anti } \\
\text { aptamer }\end{array}$ & $\begin{array}{l}\text { Fluorescence } \\
\text { Intensity }\end{array}$ & 720 & 751 & 7 & 0.00021 & $\begin{array}{l}\text { Cereal (Rice, } \\
\text { Wheat, Corn) }\end{array}$ & OTA, AFB1 & [166] \\
\hline $\begin{array}{l}\text { GONC on DEP } \\
\text { carbon electrodes }\end{array}$ & GONC & $\begin{array}{l}\text { Peak current } \\
\text { intensity }\end{array}$ & 65 & - & - & 10.82 & Tris buffer & OTA, Thrombin & [167] \\
\hline $\begin{array}{l}\text { Reduced graphene/ } \\
\text { Pt NPs micromotors }\end{array}$ & $\begin{array}{l}\text { FAM labelled } \\
\text { aptamer }\end{array}$ & Fluoresecence & 17 & 30,20 & 3,2 & 0.70 & Maize. Beer & OTA & [168] \\
\hline $\begin{array}{l}\mathrm{GO}-\mathrm{cDNA} \text { (probe1)\& } \\
\mathrm{Fe}_{3} \mathrm{O}_{4} / \mathrm{GO}-\mathrm{cDNA} \\
\text { (probe 2) }\end{array}$ & $\begin{array}{l}\text { Allochroic dyes } \\
\text { (thymolphthalein)- } \\
\text { alkaline conditions }\end{array}$ & Absorbance & 90 & 40 & 7 & $\begin{array}{l}100 \\
\text { (lowest } \\
\text { value } \\
\text { explored) }\end{array}$ & Peanut & $\begin{array}{l}\text { OTA, AFB1, } \\
\text { microcystin-LR }\end{array}$ & [169] \\
\hline $\begin{array}{l}\text { Amine funtionalized } \\
\mathrm{Fe}_{3} \mathrm{O}_{4} \text { magnetic } \\
\text { particles }\end{array}$ & $\begin{array}{l}\mathrm{NaYF}_{4}: \mathrm{Ce} / \mathrm{Tb} \\
\text { nanoparticles- } \\
\text { cDNA }\end{array}$ & $\begin{array}{l}\text { Fluorescence } \\
\text { decrease }\end{array}$ & 60 & $>2$ & 7 & 0.000019 & Maize & $\begin{array}{l}\text { OTA } \\
\text { T-2, AFB1, OTB, ZEN }\end{array}$ & [170] \\
\hline $\begin{array}{l}\mathrm{GO} / \mathrm{Fe}_{3} \mathrm{O}_{4} \\
\text { nanocomposites }\end{array}$ & Aptamer-Red QDs & $\begin{array}{l}\text { Fluorescence } \\
\text { inensity }\end{array}$ & 60 & - & 3 & 0.0162 & Peanut & $\begin{array}{l}\text { OTA, AFB1, OTB. } \\
\text { AFM1, AFB2 }\end{array}$ & [171] \\
\hline cDNA on AuE & Methylene blue & Peak current & 40 & 45 (Corn) & $\begin{array}{l}3 \text { (Corn) } \\
1\end{array}$ & 0.00015 & $\begin{array}{l}\text { Corn } \\
\text { Beer }\end{array}$ & OTA, ZEN, AFB1 & [172] \\
\hline $\begin{array}{l}\text { MoS2-Au modified } \\
\text { GCE }\end{array}$ & FC6S -Au-cDNA & $\begin{array}{l}\text { Current } \\
\text { difference }\end{array}$ & 15 & - & - & 0.0005 & PBS & $\begin{array}{l}\text { ZEN } \\
\alpha-Z O L, \quad A F B 1, D O N, \\
\text { T-2, OTA }\end{array}$ & [173] \\
\hline cDNA on AuE & AuNRs-Fc & DPV & 10 & - & 4 & & Beer & & [174] \\
\hline cDNA on AuNR & Cy5.5-aptamer & $\begin{array}{l}\text { SERS/ } \\
\text { Fluorescence }\end{array}$ & 45 & 735 & 8 & $\begin{array}{l}0.0003 / \\
0.0005\end{array}$ & Corn & $\begin{array}{l}\text { AFB1, ZEN, PAT, } \\
\text { OTA, FB2, FB3 }\end{array}$ & [175] \\
\hline $\begin{array}{l}\text { Streptavidin coated } \\
\text { microplate }\end{array}$ & TMB & Absorbance & 73 & 30 & 11 & 0.3 & $\begin{array}{l}\text { Beer } \\
\text { Corn }\end{array}$ & AFB, DON, OTA, ZEN & [176] \\
\hline cDNA2 on AuNR & $\begin{array}{l}\text { UCNPs-Hibridized } \\
\text { TAMRA-cDNA1\& }\end{array}$ & Fluorescence & 50 & 735 & 7 & 0.000003 & Corn & $\begin{array}{l}\text { ZEN, AFB1, OTA, } \\
\text { PAT, OTB }\end{array}$ & [177] \\
\hline $\begin{array}{l}\text { Aptamer-Magnetic } \\
\text { Beads }\end{array}$ & $\begin{array}{l}\text { Aptamer } \\
\text { cDNA-AgNP }\end{array}$ & $\begin{array}{l}\text { Ag intensity } \\
\text { (ICP-MS) }\end{array}$ & 121 & 42 & 8 & 0.3 & Wheat Flour & $\begin{array}{l}\text { OTA, AFB1, DON, } \\
\text { ZEN, FB2 }\end{array}$ & [197] \\
\hline $\begin{array}{l}\text { Aptamer-AuNP- } \\
\text { UCNP-AuNP-cDNA }\end{array}$ & 4-MBA & SERS & 121 & 735 & 9 & 0.00002 & Corn & $\begin{array}{l}\text { ZEN, OTA, AFB1, } \\
\text { PAT, T-2 }\end{array}$ & [198] \\
\hline ITO electrodes & $\begin{array}{l}\text { Silver-Au-Aptamer- } \\
\text { cDNA-Fe } \mathrm{O}_{4} \text { \& } \\
\text { Prussian Blue }\end{array}$ & $\begin{array}{l}\text { Color change of } \\
\text { ITO (Mobile } \\
\text { phone) }\end{array}$ & 62 & - & - & 0.01 & Corn & DON, OTA & [199] \\
\hline AuNP & AuNP & UV-Vis & 192.2 & - & - & 0.000056 & $\begin{array}{l}\mathrm{MgCl}_{2} 1 \mathrm{mM} \\
\text { Buffer }\end{array}$ & OTA, AFB1 & [200] \\
\hline
\end{tabular}

1Abbreviations: AFB1: Aflatoxin B1; AFB2: Aflatoxin B2; AFG1: Aflatoxin G1; AFG2: Aflatoxin G2; AgNP: Silver nanoparticles; AuE: Gold electrode; AuNP: Gold nanoparticles; AuNRs: Gold
nanorods; BHQ2: Black hole quencher; cDNA: complementary DNA; CTN: Citrinin; Cy: Cyanine; DEP: Disposable electrical printed; DON: Deoxynivalenol; DPV: Differential pulse voltammetry; FAM: Fluorescein amidine; FB2: Fumonisin B2; Fc: Thiol modified ferrocene; FC6S: 6-(Ferrocenyl)hexanethiol; FITC: Fluorescein isothiocyanate; GCE: Glassy carbon electrode; GO: Graphene oxide; GONC: Graphene oxide nanocolloids; GS: Graphenes; ICP-MS: Inductively coupled plasma mass spectrometry; ITO: Indium Tin Oxide; MB: Molecular beacon; MBA: Mercaptobenzoic acid enhanced Raman spectroscopy; SPCE: Screen-printed carbon electrode; SPCM: Silica photonic crystal microsphere; TAMRA: Carboxytetramethylrhodamine; TH: Thionine; TMB: $3,3^{\prime}, 5,5^{\prime}$ tetramethylbenzidine; UCNPs: Upconversion fluorescent nanoparticles; ZEN: Zearalenone; ZOL: Zearalenol; PAT: Patulin tetramethylbenzidine; UCNPs:
${ }^{2}$ Mycotoxins highlighted in bold indicate a multiplex assay 
a)

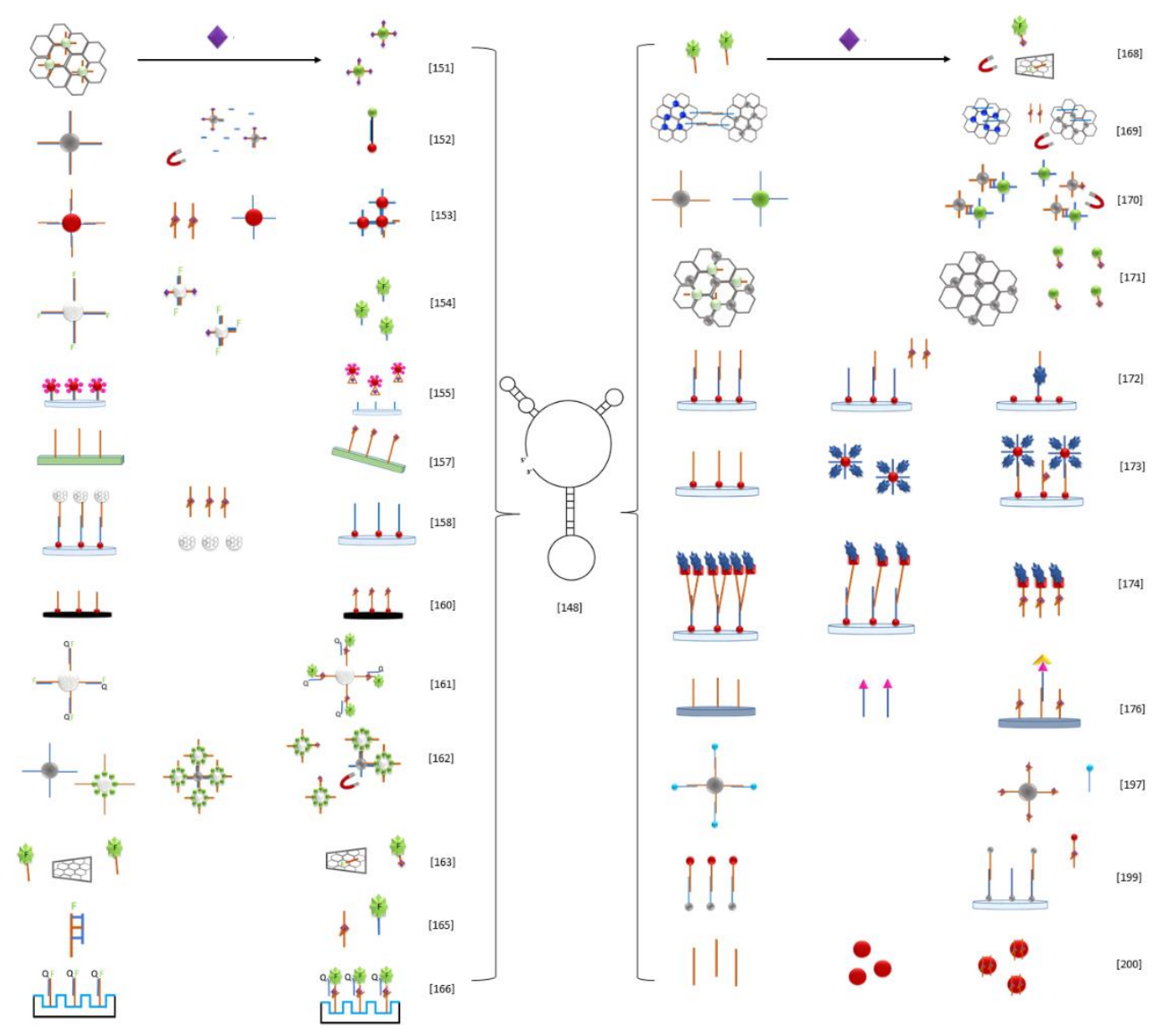

b)

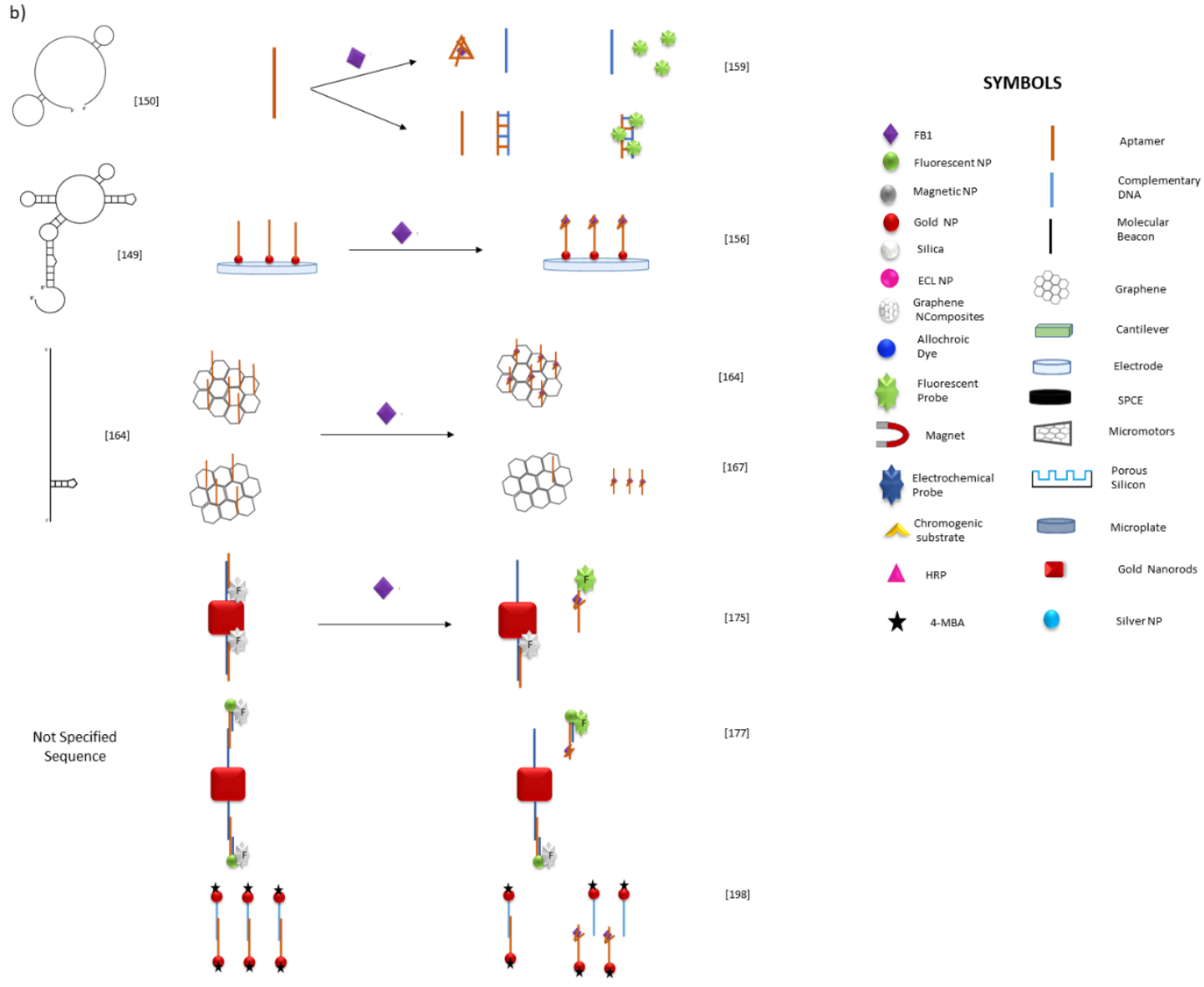

Figure 6. Schematic representation of the various mechanisms of aptamer-based biosensors with (a)the $96 \mathrm{nt}$ aptamer and (b)other shorter or not specified sequences. (Abbreviations: ECL: Electrochemiluminescence; HRP: Horseradish peroxidase; MBA: Mercaptobenzoic acid, NP: Nanoparticle; SPCMs: Silica photonic crystal microspheres) 


\subsection{A 96-mer aptamer for fumonisin determination}

The first aptamer specific for FB1 was reported by McKeague [148], after 18 SELEX rounds through negative selections with unmodified and modified (L-homocysteine, L-cysteine, L-methionine and L-glutamic acid) magnetic beads. From the six sequences initially studied, the sequence with the lowest $G$ content ( $8 \%$ ) was selected due to its greatest binding affinity, confirmed by its low dissociation constant $(\mathrm{Kd}=100 \mathrm{nM})$. This sequence consisted in 60 random nucleotides (bold letters), surrounded by two primer binding sites: 5'-ATA CCA GCT TAT TCA ATT AAT CGC ATT ACC TTA TAC CAG CTT ATT CAA TTA CGT CTG CAC ATA CCA GCT TAT TCA ATT AGA TAG TAA GTG CAA TCT-3'.

\subsubsection{Fluorescent detection}

From all the biosensing designs applying the $96 \mathrm{nt}$ aptamer, the most sensitive were those transduced into fluorescent $[154,166,170]$ and electrochemical $[172,173,174]$ signals. The first fluorescent method described the application of avidin-modified fluorescent nanoparticles and graphene oxide (GO), as donor/acceptor pair in Förster resonance energy transfer (FRET). A biotin modified aptamer was attached to upconversion fluorescent particles (UCNPs); under the presence of an increasing fumonisin concentration, the particles were not quenched by $\mathrm{GO}$, thus exhibiting a linear increment on the fluorescence intensity [151]. The surface of reduced GO/platinum nanoparticles (PtNPs) and RGO/Ni/PtNPs micromotors were also used as a quencher of fluorescein amidine (FAM)-aptamers, with a direct increase on its fluorescence intensity produced by target biding $[163,168]$. A similar procedure was proposed for the FRET-quenching effect between AuNP and UCNPs attached to a molecular beacon (MB), although the measured signal intensity was an indirect analysis of the fumonisin content. To this end, a biotinylated aptamer (linked to avidin modified magnetic particles), was hybridized with its complementary DNA, which was also able to hybridize and open the MB, thus forming a fluorescent double-stranded DNA arrangement [152].

In the most sensitive development with this long sequence, the functionalization of magnetic nanoparticles with aptamers and time-resolved fluorescent nanoparticles with complementary DNA, resulted in the formation of capture and signal probes, respectively. The DNA hybridization step derived to a magnetic/fluorescent biocomplex, whose magnetic separation at rising concentrations of FB reported a reduction in fluorescence intensity [170]. Similarly, amino modified aptamers hybridized with their fluorescein isothiocyanate (FTIC)- modified complementary DNA, were coupled to silica photonic crystal microspheres (SPCMs), with an inhibitory effect on the fluorescent signal caused by an increasing target concentration [154]. In fact, good sensitivity can be achieved with SPCM suspensions, when bound to a hybridized duplex structure formed by a black hole quencher (BHQ2)labelled antiaptamer (quencher) and a $\mathrm{NH}_{2}\left(5^{\prime}\right) / \mathrm{Cy} 3\left(3^{\prime}\right)$ modified aptamer. An increasing FB1 concentration enlarged the separation between the dye and its corresponding quencher, promoting a higher fluorescent signal [161]. Moreover, the hybridization between a $\mathrm{NH}_{2} / \mathrm{Cy} 3$ modified aptamer and its $\mathrm{BHQ} 2$-antiaptamer was examined when immobilized to a $\mathrm{TiO}_{2}$ modified silicon wafer, where the increment of fluorescence was triggered by the addition of FB1 [166]. Unlike other techniques, a less sensitive electrochemiluminescent (ECL) assay resulted from AuNP modified with a thiolated aptamer and an iridium complex, when fixed to an Au electrode by a partial complementary DNA. As the AuNP/lr complex enhanced the electrode conductivity, the addition of FB1 decreased its ECL signal [155]. A very sensitive aptasensor for FB1 combined the interaction of a biotinylated aptamer and its biotinylated complementary DNA, conjugated to magnetic and NaYF4:Ce/Tb nanoparticles respectively. The addition of the target mycotoxin produced a decrease on the complex formation, therefore a signal decrease on the resulting fluorescence [170].

\subsubsection{Electrochemical detection}

As already stated, electrochemical methods have also been applicable to sensitive FB1 aptasensors, and their precise completion can be enhanced by the addition of materials such as AuNP and graphene-thionine (GS-TH). Electrodes functionalized with AuNP are convenient for DNA attachment, and the complexity of its fabrication depends on the aptamer structure. For instance, the unmodified 96-mer molecule was docked to a AuNP modified glassy carbon electrode through a 
thiolated capture DNA. A higher sensitivity was promoted by the addition of GS-TH, due to its competition against FB1 for binding the aptamer, which also generated a decrease in the redox peak [158]. Efforts for reducing the costs and increasing the capacity of aptasensors have focused on a combination of powerful electrochemical techniques with portable devices. A screen-printed carbon electrode (auxiliary, reference and working electrodes included) modified with polydimethylsiloxane was selected for the electrodeposition of AuNP, and further attachment of a thiolated aptamer. The coil to G-quadruplex conformational transition, supported by the presence of FB1, was applied to strengthen the electron transfer resistance, reflected as a reduction in the electrochemical impedance spectroscopy response [160]. While the previous methods were able to quantify FB1 in a $\mathrm{ng} / \mathrm{L}$ scale, upcoming electrochemical assays are reaching limits of detection in the $\mathrm{pg} / \mathrm{L}$ range.

Gold electrodes worked as ideal supports for combined DNA structures, as verified for doublestranded DNA (aptamer-cDNA). The incubation with different concentrations of FB1 in this assay left some free and hybridized cDNA that had to be subsequently washed with exonuclease I. The remaining double-stranded DNA interacted with methylene blue whose electrochemical signal reached a LOD of $0.00015 \mu \mathrm{g} / \mathrm{L}$ [172]. A Y-shaped hybridized structure was also conjugated on a gold electrode. This approach included a DNA sequence complementary on different segments to two aptamers and the addition of gold nanorods for signal enlargement related to concentrations of FB1 as low as $0.00026 \mu \mathrm{g} / \mathrm{L}$ [174]. Another technique in the $\mathrm{pg} / \mathrm{L}$ scale $(0.0005 \mu \mathrm{g} / \mathrm{L})$ was designed on a glassy carbon electrode modified with molybdenum disulfide and gold nanoparticles for the attachment of aptamers and further immobilization with labelled cDNA, whose differential pulse voltammetry (DPV) decreased with the addition of FB1 [173].

\subsubsection{Alternative and colorimetric detection}

Alternative aptasensors comprised gold-modified microcantilevers, capable of containing thiolated aptamers, in which the differential deflection linearly increased with higher FB1 concentrations [157]. In a different novel method, $\mathrm{Fe}_{3} \mathrm{O}_{4} @ \mathrm{Au}$ magnetic beads were also coupled with a thiolated complementary DNA, for the hybridization of amino-modified aptamers, conjugated with $\mathrm{SiO}_{2} @ \mathrm{PbS}$ hybrid spheres. An increasing concentration of FB1 produced a reduction on the number of hybridized labels, which after a magnetic separation were dissolved in acid for the square wave voltammetry of the remaining $\mathrm{Pb}^{2+}$ [162]. As already illustrated, magnetic beads promoted the easy separation of their unhybridized attached sequences, which was also reported for the fluorometric test of a FAM labelled complementary DNA displaced from its hybrid form with its corresponding aptamer, upon FB1 binding [165]. A similar approached was proposed with aptamer functionalized with magnetic beads, whose hybridization with cDNA-silver nanoparticles (AgNPs) was diminished by the presence of target, with further inductively coupled plasma mass spectrometry of Ag released as cDNA-AgNPs [197].

In order to reduce the complexity of the assays, three colorimetric methods have been proposed for the unmodified version of this aptamer. On the first system, gold nanoparticles were functionalized with either a thiolated short-strand (DNA1) complementary to the unmodified aptamer or a thiolated short-strand complementary to DNA1 (DNA2). The association of the aptamer and DNA1 was interrupted by the addition of FB1, which also permitted the hybridization of AuNP-DNA1 and AuNPDNA2, causing aggregation and color shift from red to blue [153]. For the second approach, thymolphthalein was adsorbed on the surface of GO nanoparticles modified with a semi complementary DNA. The use of the unmodified 96-mer aptamer as a DNA linker, allowed the conjugation of the labelled $\mathrm{GO}$ with $\mathrm{Fe}_{3} \mathrm{O}_{4} / \mathrm{GO}$, previously modified with a second semi complementary DNA. After target incubation, the $\mathrm{Fe}_{3} \mathrm{O}_{4} / \mathrm{GO}$ particles were magnetically removed, and a colorimetric detection was revealed by adjusting the $\mathrm{pH}$ of the remaining solution containing labelled GO [169]. Another colorimetric assay was proposed through the competition between a HRPcDNA and FB1 for binding an aptamer immobilized on a streptavidin-coated microplate. Depending on the amount of FB1, a colorless TMB solution was catalyzed by the hybridized HRP-cDNA to obtain the blue oxTMB, whose yellow color was exposed by the stopping solution (sulfuric acid) [176]. A more complex colorimetric method was design through a glassy carbon electrode (GCE) modified with $\mathrm{Fe}_{3} \mathrm{O}_{4}-\mathrm{cDNA}$-aptamer-AuNP at different degrees, due to dehybridization by FB1. Such sensing electrode was connected to a Pt wire through an electric bridge, where varying target concentrations 
resulted in different GCE effective areas and current flow, reported as deposition of Prussian blue (PB) on an ITO electrode. This indirect electrochemical analysis was translated into a colorimetric signal by means of the smartphone detection of deposited PB at the ITO electrode, submerged in the reporting solution along with the Pt wire [199]. In contrast to previous reports, the sole application of the unmodified sequence ( $96 \mathrm{nt}$ ) was reported by our research group in an aptamer-FB1-AuNPs conjugate, stable to salt-induced aggregation at an increasing target concentration, under the presence of $\mathrm{MgCl}_{2}$ [200].

\subsection{Shorter sequences and minimers derived from the $96 \mathrm{nt}$ aptamer}

Five years after the dissemination of the first aptamer specific to FB1 [148], the same research group explored the affinity of minimers (truncated aptamers) from the initial $96 \mathrm{nt}$ aptamer. The different structures included the whole sequence, and its subsequent chains created by preserving the 3' stem loop motif, removing the 3', 5', or both primer binding regions (PBR).

Larger melting temperatures from minimers containing the 3' region, suggested their role on the stability and complete formation of hairpins [150]. The same study compared the binding affinity through the calculation of the dissociation constant $(\mathrm{Kd})$ by two assays: DNase I and magnetic beads. The DNase I assay indicated similar affinities between the minimer without the two PBR and the fulllength oligonucleotide (Table 6); however, this method also carried considerable errors and denoted binding towards FB2. On the other hand, the magnetic beads confirmation assay proved the high affinity of minimers lacking the $3^{\prime}$ and both PBR, as well as their overall upgraded binding, due to primary amine masking by the beads, suggesting a most favorable interaction with the tricarballylic acid regions [150]. A reduction on the sequence length might lead to the development of simpler, yet more sensitive biosensors. The interaction within the shorter 60 nt strand without PBR and its complementary DNA was tested under the presence of different concentrations of FB1, in which the rate of double-stranded DNA formation was identified with the fluorescent dye PicoGreen [159]. Regardless of the specificity issues presented by Frosts [150], the truncated sequence studied by Gui [159] was capable of discriminating ochratoxin A (OTA) aflatoxin B1 (AFB1), citrinin (CTN) and zearalenone (ZEN), while the specificity of the original long length aptamer was already confirmed for the null interaction with OTA, AFB1, AFB2, AFG1, AFG2, FB2, ZEN, L-cysteine, BSA, T-2 toxin and deoxynivalenol (DON)(Table 5). Still, even when this 60 nt aptamer-based method was correlated to a reduction on the assay and assay preparation times, its depicted LOD was higher than the values achieved with the full $96 \mathrm{nt}$ sequence.

\subsection{A novel oligonucleotide for the determination of FB1}

Four years after the first reported sequence, a new aptamer selection was presented by using a library of single stranded DNA designed with 80 nt sequences, in which 40 random nucleotides (bold letters) were edged by $20 \mathrm{nt}$ on each side. The SELEX process was executed with the aid of magnetic beads, and included negative (magnetic beads), positive (FB1 modified magnetic beads) and counter (free glycine, AFB1, AFB2, ZEN) selection rounds, which also served to confirm the aptamer selectivity. The selected aptamer: 5'-AGC AGC ACA GAG GTC AGA TG C GAT CTG GAT ATT ATT TTT GAT ACC CCT TTG GGG AGA CAT CCT ATG CGT GCT ACC GTG AA-3, showed a lower Kd (62 $\mathrm{nM}$ ), hence a greater affinity to FB1 was expected for the development of more sensitive aptasensors than that with the 96 nt aptamers [149]; however, this was not the case and the aptasensors so far reported using this aptamer have not shown the expected superior sensitivity, which was also confirmed by its fewer applications.

After its introduction, the full-length thiolated version was docked on glassy carbon electrodes in order to enhance its electron transfer resistance, whose decrement was caused by the addition of the target mycotoxin [156]. This electrochemical arrangement derived in a sensitive method, with a similar LOD to previous electrochemical aptasensors for FB1 [157, 160]. Furthermore, a shorter version, consisting on its 40 random nucleotides, was casted on doped ( $\mathrm{B}$ or $\mathrm{N}$ ) and undoped graphene modified GCE, from which boron-doped graphene helped immobilize a higher amount of FB1, improving the impedimetric signal thus the sensitivity of the electrochemical sensor [164]. This $40 \mathrm{nt}$ aptamer was also immobilized on graphene oxide nanocolloids (GONCs), causing a reduction on the electroactivity from the oxygen containing groups. The addition of FB1 prompted the full detachment 
of the aptamer and the partial reestablishment of electroactivity, with potential for biosensing purposes and verified sensitivity under the presence of OTA and thrombin [167]. Although the latter corresponded to low assay and assay preparation times, both biosensors were not comparable to the applications with longer chains. Further research is needed to reveal the affinity mechanism for this aptamer to understand its sensitivity constrains and fully develop highly sensitive aptamer-based sensors.

\subsection{Not specified sequences and alternative methods}

Three studies published by the same research group did not specify the aptamers sequence for the detection of FB1. The first approach relied on the hybridization of Cy5.5-aptamer and its cDNA on gold nanorods, with a further measurement of their SERS (LOD: $0.0003 \mu \mathrm{g} / \mathrm{L}$ ) and fluorescent (LOD:0.0005 $\mu \mathrm{g} / \mathrm{L}$ ) signals under the presence of the target mycotoxin [175]. The second work, which so far is the most sensitive aptasensor for FB1, was reported with a LOD of $0.000003 \mu \mathrm{g} / \mathrm{L}$. In this arrangement, the inner filter effect between UCNPs and gold nanorods, both linked by a hybridized aptamer, was reduced by disrupting the biocomplex through target incubation and stimulating fluorescence under excitation $(980 \mathrm{~nm})$ [177]. The third biosensor combined the modification of AuNPs with aptamers and 4-mercaptobenzoic acid as a Raman reporter, whose signal was reduced after target incubation through dehybridization from a cDNA-AuNP-(4-MBA) complex, with an LOD of $0.00002 \mu \mathrm{g} / \mathrm{L}$ [198]. The effect of the electrochemical interaction between FB1 and fish sperm doublestranded DNA was examined on the impedimetric detection with a pencil graphite electrode, which provides a promising biosensing technique with other DNA structures apart from aptamers [178]. Nevertheless, the addition of five FB1 concentrations did not portray differentiated responses; therefore, more optimization would be ideal for the application of this type of non-specific sequences.

\subsection{Multiplex detection}

Aptasensors are not restricted to the sole determination of single mycotoxins, multiplex analysis can be accomplished with different arrays. Fluorescent [151] and magnetic [162, 197] nanoparticles, as well as their association [170,171], were applied for the multiple detection of FB1 and OTA. Moreover, photonic crystal microspheres were able to support double (FB1, OTA) and triple (FB1, OTA, AFB1) mycotoxin quantification [154, 161]. In a similar way to fluorescent particles, the application of fluorescent labels favored the establishment of optimum $\lambda_{\mathrm{em}}$ in combination with their specific reading methods (filters), for the detection of FB1 and OTA [163]. The specific allocation of a cy3 aptamer and its $\mathrm{BHQ}$ antiaptamer on $\mathrm{TiO}_{2}$ modified silicon wafers, was also suitable for the linear quantification of multiple mycotoxins (OTA, AFB1, FB1), where the fluorescence increment was spotted on a defined area of a wafer surface [166]. The combination of two different fluorescent compounds with UCNPs induced two resolved responses under the presence of ZEN and FB1 [177], while the functionalization of UCNPs and AuNPs with aptamers along with aptamer labelling were exploited in the multiplex SERS and fluorescence detection of ZEN, OTA and FB1, through a triple hybridization with a cDNA-AuNPs complex [198]. Likewise, as previously mentioned, the combination of different allochroic dyes with magnetic and GO nanoparticles, was also convenient for the colorimetric detection of FB1, OTA, AFB1 and microcystin-LR [169]. 
Table 6 DNA sequences utilized for different aptasensor and their binding conditions ${ }^{1}$

Aptamer Modification cDNA Other

Binding Buffer

\section{5'-ATA CCA GCT TAT TCA ATT AAT CGC ATT ACC TTA TAC CAG CTT ATT CAA TTA CGT CTG CAC ATA CCA GCT TAT TCA ATT AGA TAG TAA GTG CAA TCT-3'3}

5'-Biotin-(CH2)6-

Tris-HCl buffer (10 mM containing $100 \mathrm{mM} \mathrm{NaCl,} \mathrm{pH} 7.4)$

$37^{\circ} \mathrm{C}$ Overnight (conjugation in BB)

$37^{\circ} \mathrm{C}, 2 \mathrm{~h}$ (Binding)
$37^{\circ} \mathrm{C}, 80$ min (Incubation with GO)

5'-Biotin-(CH2)6-

5'-AAT TGA ATA AGC TGG-3

Molecular Beacon $10 \mathrm{mM}$ PBS

None

5' -SH-AAT TGA ATA AGC TGG TA-3'

5'-SH TAC CAG CTT ATT CAA TT-

$-(\mathrm{CH} 2) 6-\mathrm{NH}_{2}-3^{\prime}$

5'-FITC-AAT TGA ATA AGC TGG TA-3

5'-SH-(CH2)6-

-SH-(CH2)6-AAT TGA ATA AGC TGG TAT

5'-SH-(CH)6-

None

5'-SH-(CH2)6-AAT TGA ATA AGC TGG TA-3

5'-AAT CGC ATT ACC TTA TAC CAG CTT ATT CAA

5'-AAT TGA ATA AGC TGG TAT GTG CAG ACG TAA TTG AAT

FB139t3: F- ATA CCA GCT TAT TCA ATT AAT CGC ATT ACC TTA TAC CAG CTT ATT CAA FB13933-5: F- AAT CGC ATT ACC TTA TAC CAG CTI ATT CAA TIA CGT CTG CAC ATA CCA GCT TAT TCA ATT
5'-SH-(CH2)6-

$37^{\circ} \mathrm{C} 12 \mathrm{~h}$ (immobilization on MNPs)
$37^{\circ} \mathrm{C}, 30$ min (hibridization aptamer-cDNA)

$37^{\circ} \mathrm{C}, 30-40$ min (incubation)

$37^{\circ} \mathrm{C}$, shaking for $12 \mathrm{~h}$ (funtionalization)

$10 \mathrm{mM} \mathrm{PB}$ containing $1 \%$ SDS by mass $\mathrm{pH} 7.4$ (DNA dilution)

$500 \mathrm{Mm} \mathrm{NaCl} \mathrm{CDNA}$

$1 \times$ PCR amplification buffer (Conjugate dilution)

$20 \mathrm{mM} \mathrm{NaCl}+10 \mathrm{mM}$ PB

$95^{\circ} \mathrm{C}, 5$ mint (hibridiization cDNA1-cDNA2)

TE solution (100 mM Tris-HCl + $10 \mathrm{mM}$ EDTA) $5 \times$ saline sodium citrate (hibridization)
$10 \mathrm{mM}$ Tris-HCl (pH 8.0), $120 \mathrm{mMM} \mathrm{NaCl}, 20 \mathrm{mM} \mathrm{CaCl} 2,5 \mathrm{mM} \mathrm{K}$
$20 \mathrm{mM} \mathrm{MgCl}$ (binding)

$4^{\circ} \mathrm{C}, 12 \mathrm{~h}$. (Immobilization on SPCMs in TE solution)

$4^{\circ} \mathrm{C}, 12 \mathrm{~h}$. (Immobilization on SPCMs in
$37^{\circ} \mathrm{C}, 1$, (blocking with $1 \mathrm{~B} \% \mathrm{BSA}$ PBS)

$37^{\circ} \mathrm{C}, 2$. 2 . (hibridization)
$37^{\circ} \mathrm{C}, 1 \mathrm{~h}$ (binding)

Methanol 50\%

$80^{\circ} \mathrm{C}, 5 \mathrm{~min}$ (hibridization)

Cooled to $\mathrm{RT}$
$37^{\circ} \mathrm{C}, 2 \mathrm{~h}$ (binding)

$10 \mathrm{mM}$ Tris- $\mathrm{HCl}, 100 \mathrm{mM} \mathrm{NaCl}$,

(bo mM TCEP, pH 7.4 (immobilization)

$3 \mathrm{~h}, 25^{\circ} \mathrm{C}$ (Functionalization)

$1 \mathrm{~h}, 25^{\circ} \mathrm{C}$ with $\mathrm{MCH}$ (blocking)

$24 \mathrm{~h}, \mathrm{RT}$ (cDNA immobilization)

$10 \mathrm{mM}$ Tris-HCl buffer $\mathrm{pH} 7.4$ (hibridization)

PBS (pH 7.4). (binding)

0
0 $10 \mathrm{mmol} / \mathrm{L}$ Tris, $120 \mathrm{mmo} / \mathrm{L} \mathrm{NaCl}, 5 \mathrm{mmo} / \mathrm{L} \mathrm{KCl} 、 20 \mathrm{mmo} / \mathrm{L}$

oom temperature, 25 min (binding)

$100 \mathrm{mM} \mathrm{NaCl}, 20 \mathrm{mM}$ Tris, $2 \mathrm{mM} \mathrm{MgCl}, 5 \mathrm{mM} \mathrm{KCl}, 1 \mathrm{mM} \mathrm{CaCl}$,

$95{ }^{\circ} \mathrm{C}, 5 \mathrm{~min}$ (denaturation)

$25^{\circ} \mathrm{C}, 20$ min (Incubation)

$25^{\circ} \mathrm{C}, 5 \mathrm{~min}$ (hibridization)

DNase I assay: 30 min, RT (Incubation with FB1)

Magnetic beads assay:

RT, 60 min (Incubation)

Aptamer stock: $50 \mathrm{mM}$ Tris-HCl buffer (ph 7.4, $0.1 \mathrm{M} \mathrm{NaCl}, 0.2 \mathrm{M}$

$\mathrm{KCl}, 5 \mathrm{mMM} \mathrm{MgCl}$ and $1 \mathrm{mM}$ EDTA)
Activation Buffer: $50 \mathrm{mM}$ Tris-HCl with $100 \mathrm{mM}$ TCEP

Room temperature, $1 \mathrm{~h}$ (activation)

$6 \mathrm{~h}$ and $4{ }^{\circ} \mathrm{C}$ (SPCE modification with activated aptamer)

(10.4 Binding buffer: TE buffer containing $0.1 \mathrm{M} \mathrm{NaCl}, 0.2 \mathrm{M} \mathrm{KCl}$, and

h, RT (Blocking with MCH)

Binding buffer: Tris- $\mathrm{HCl}, 0.01 \mathrm{M}, \mathrm{pH} 8.0, \mathrm{NaCl} 120 \mathrm{mM}, \mathrm{CaCl}_{2} 20$ $\mathrm{mM}, \mathrm{KCl}, 5 \mathrm{mM}, \mathrm{MgCl}_{2} 20 \mathrm{mM}$

60 min and $37^{\circ} \mathrm{C}$ (hibridization) Room Temperature $12 \mathrm{~h}$ (Immobilization on microspheres)

$10 \mathrm{mMTris} H \mathrm{HCl} 1 \mathrm{mM}$ EDC $1 \mathrm{mMNHS}$ (aptamer coniugation) $10 \mathrm{mM}$ Tris-HCl with $100 \mathrm{mM}$ TCEP (CDNA activation)

$37^{\circ} \mathrm{C}, 1 \mathrm{~h}$. (cDNA activation)

$37^{\circ} \mathrm{C}, 30$ min (CDNA incubation with MBs

$37^{\circ} \mathrm{C}, 2 \mathrm{~h}$. (hibridization)
$37^{\circ} \mathrm{C}, 1 \mathrm{~h}$ (binding) 
5'-FAM-

5'-NH2-(CH2)6- reverse sequence-Cy3-3

FAM- AATAAGCTGGTATGT

5'-FAM-

None

5'-biotin-(CH2)6-

$-\mathrm{NH} 2-3^{\prime}$

None

5'-SH-(CH2)6

5'-SH-

5'-biotin

'-biotin

5'SH-C6-

5'AGC ACCA

5 -SH-(CH2)6-

5'C GAT CTG GAT ATT ATT TTT GAT ACC CCT

TTG GGG AGA CAT- 3

5'-C GAT CTG GAT ATT ATT TTT GAT ACC CCT
TTG GGG AGA CAT- $3^{\prime}$

NOT SPECIFIED SEQUENCES CAC TTA CTA TGG TAT GTG CAG ACG TAA-3
5'-BHQ2-TAT GGT CGA ATA AGT TAA-3'

5'-TTG AAT AAG CTG GTA TAA
GGT AAT GCG ATT AAT TGA ATA GGT AAT GCG ATT AAT TGA ATA
AGC TGG TAT GTG TGT GTG TGT
GTG TGT GTG TGT GTG TGT-3,

5-GTG TGT GTG TGT GTG TGT GTG TGT GTG TGT AGA
CAC TTA CTA TCT AAT TGA ATA AGC TGG TAT GTG CAG

5'-biotin--(CH2)6-TCT AAT TGA ATA AGC TGG TAT GTG CAG
ACG-3'

5'-SH-GAG GGG TGG GCG GGA GGG AGA TTG CAC GGA CTA

${ }_{3}^{\prime}$-SH-(CH2)6-AATTGAATAAGCTGG

5'-SH-GAG GGG TGG AGA TTG CAC TTA CTA TCT AAT TGA

5'-biotin- AGA TTG CAC TTA CTA TCT AAT TGA ATA AGC TGG 5-biotin- AGA CAG ACG TAA TTG AAT AAG CTG GTA TAA GGT
AAT GCG ATT AAT TGA ATA AGC TGG TAT - 30 .

5'-biotin-GAT AGG AGT CGT GTG GGA TAG

TGT GGG AGA TTG CAC TTA CTA TCT AAT TGA ATA AGC

5-NH2-C6-AAT TGA ATA AGC TGG TA-3'

\section{ATT ATT TTT GAT ACC CCT TTG GGG AGA CAT CCT ATG CGT GCT ACCC GTG AA-3}

PBS (10 mM Na2 $\mathrm{HPO}_{4}, 137 \mathrm{mM} \quad \mathrm{NaCl}, 2.7 \mathrm{mM} \mathrm{KCl}, 2 \mathrm{mM}$
Tris-HCl pH 7.5; $10 \mathrm{mM}$

(pH 7.5) with $0.01 \%$ Tween (Aptamer dilution)

$20 \mathrm{mM}$ Tris, $0.1 \mathrm{M} \mathrm{NaCl}$,
$\mathrm{pH} 7.6$ (Binding buffer)

Binding buffer: Tris-HCl $10 \mathrm{mM}\left(\mathrm{pH}\right.$ 8.0), $\mathrm{NaCl} 120 \mathrm{mM}, \mathrm{CaCl}_{2} 20$

$10 \mathrm{mM}$ Tris-HCl pH 7.5 (aptamer reconstitution/ incubation)

SDS $1 \% \mathrm{v} / \mathrm{v}$ (aptamer capture)

PBS $100 \mathrm{mM} \mathrm{pH} 7.5$ with Milli-Q water and $0.01 \%$ of Tween (PBS-

T) (Aptamer dilution)
PBS, (Na2 $\mathrm{HPO}_{4}-\mathrm{NaH}_{2} \mathrm{PO}_{4}, 0.1 \mathrm{M}$ )

$25^{\circ} \mathrm{C}, \quad 15$ min (Incubation)

$95^{\circ} \mathrm{C}, 5$ min (Heating)

5 min on ice
$37^{\circ} \mathrm{C}, 1 \mathrm{~h}$. (hibridization)

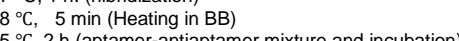

$37^{\circ} \mathrm{C}, 12 \mathrm{~h}$ (hibridizaztion-immobilization)

$37^{\circ} \mathrm{C}, 12 \mathrm{~h}$ (Binding)

$25^{\circ} \mathrm{C}, 15$ min (Incubation)
RT, 2 min (Aptamer capture)

RT, $2 h$ (DNA1 binding on GO)

mobilization on $\mathrm{Fe} 3 \mathrm{O} 4 / \mathrm{GO}$

$37^{\circ} \mathrm{C}, 1.5 \mathrm{~h}$ (Incubation)
(n)

$37^{\circ} \mathrm{C}, 1 \mathrm{~h}$ (Incubation)

RT, overnight (bio-probe)

RT, overnight (Immobilization)
$37^{\circ} \mathrm{C}, 1 \mathrm{~h}$ (Incubation)

Tris-HCl buffer (containing $0.05 \mathrm{M}$ Tris, $0.2 \mathrm{M} \mathrm{NaCl}$ and $0.001 \mathrm{M}$

$37^{\circ} \mathrm{C}$ (cDNA Immobilization)
$37^{\circ} \mathrm{C}, 2 \mathrm{~h}$ (hibridization)

$37^{\circ} \mathrm{C}, 2 \mathrm{~h}$ (hibridization)
$37^{\circ} \mathrm{C}, 10 \mathrm{~min}$ (Incubation FB1)

$95^{\circ} \mathrm{C}, 5 \mathrm{~min}$ (Heating)

$37^{\circ} \mathrm{C}, 2 \mathrm{~h}$ (Ap conjugation to electrode)

$37^{\circ} \mathrm{C}, 2 \mathrm{~h}$ (hibridization)

15 min (Incubation)

$2 \mathrm{~h}$ (Conjugation to AunRs)
$37^{\circ} \mathrm{C}, 2 \mathrm{~h}$ (cDNA Immobilization on electrode)

$37^{\circ} \mathrm{C}, 2 \mathrm{~h}$ ( ChDA (hibridization)

$50 \mathrm{mM}$ Tris-HCl

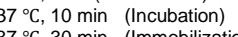

BSS buffer (10 mmo/L Na $\mathrm{HPO}_{4}, 2 \mathrm{mmol} / \mathrm{KH} \mathrm{KH}_{2} \mathrm{PO}_{4}, 2.7 \mathrm{mmol}$ $\mathrm{KCl}, 137$ mmol/L NaCl, $\mathrm{pH} 7.4$ )
$\mathrm{PBS}-\mathrm{T}$ (Washing)

$\begin{array}{ll}37^{\circ} \mathrm{C}, 30 \mathrm{~min} & \text { (Immobilization) } \\ 25^{\circ} \mathrm{C}, 60 \mathrm{~min} & \text { (Immobilization) }\end{array}$

Tris-HCl buffer $20 \mathrm{mmol} / \mathrm{L}$ with $0.5 \mathrm{mo} / \mathrm{L} \mathrm{NaCl}, 1 \mathrm{mmo} / \mathrm{L}$ EDTA

(Washing)
$20 \mathrm{mM}$ Tris-HCl pH 7.4 (Dissolving/Target Incubation)
$100 \mathrm{mM} \mathrm{Tris}-\mathrm{HCl}$ pH 7.4 (Re-dispersion)

$37^{\circ} \mathrm{C}, 120 \mathrm{~min}$ (Functionalization of magnetic beads)

$37^{\circ} \mathrm{C}, 90 \mathrm{~min}$ (Labelling of Ag NPs)

(T)

PBS, pH 7.4, 1 ( Washing, redispersion, AuNP stability)

$\mathrm{pH} 7.4$ (Hibridization, target incubation)

$37^{\circ} \mathrm{C}, 120$ min (Functionalization of $\mathrm{Fe}_{3} \mathrm{O}$

$\begin{array}{ll}37^{\circ} \mathrm{C}, 30 \mathrm{~min} & \text { (Hibridization) } \\ & \text { (Target Incubation) }\end{array}$

pH 7.4, $100 \mathrm{mM} \mathrm{NaCl}, 20 \mathrm{mM}$ Tris- $\mathrm{HCl}, 2 \mathrm{mM} \mathrm{MgCl} 2,5 \mathrm{mM} \mathrm{KCl}$,
$1 \mathrm{mMCaCl} 2$

$37^{\circ} \mathrm{C}, 6 \mathrm{~h}$ (electrode modification) $94^{\circ} \mathrm{C}, 5 \mathrm{~min}$ followed by 15 min cooling with ice

PBS pH 7.0 (aptamer solution)
Tris buffer pH 8.2(FB1 solution)

(folding)
Room temperature, $30 \mathrm{~min}$ (binding)

Room temperature, 30 min (binding)
$60^{\circ} \mathrm{C}, 15 \mathrm{~min}$ (aptamer dropcasting
$37^{\circ} \mathrm{C}, 30 \mathrm{~min}$ (Incubation)

$60^{\circ} \mathrm{C}, 10 \mathrm{~min}$ (cast on GONC)

Aptamer dilution: PBS $(10 \mathrm{mM}$

Aptamer dilution: $\mathrm{PBS}$ (aCl; $\mathrm{mM}$.2)
FB1 dilution: Tris (25 mM Tris; $300 \mathrm{mM} \mathrm{NaCl}$; pH 8.2) 
RT, 12 h (Addition of CDNA1 to aptamer-UCNPs)

$37^{\circ} \mathrm{C}, 50 \mathrm{~min}$ (Cooling)
$37^{\circ} \mathrm{C}, 50 \mathrm{~min}$ (Incubation)

$37^{\circ} \mathrm{C}, 12 \mathrm{~h}$ (Hibridization)

Hybridization buffer (not specified)

$50 \mathrm{mM}$ TE buffer $\mathrm{pH} 7.4$ ( $\mathrm{pH}$ adjustement)

(Target Incubation) 
Recent improved electrochemical methods also allowed multiplex analysis, as in the case of glassy carbon electrodes modified with enhancers of electron mobility such as $\mathrm{MoS}_{2}$ and AuNP. These were utilized for the simultaneous quantification of FB1 and ZEN produced by the different reduction peaks from FC6S and thionine, respectively, which functioned as labels for CDNA when simultaneously immobilized on colloidal gold [173]. Likewise, gold electrodes modified with a Yshaped DNA conformation were efficient for detecting OTA and FB1 due to immobilization of thiolated thionine and ferrocene on gold nanorods, which in addition of enhanced electron transfer, exhibited distinctive peak currents [174].

\subsection{Aptamer folding and aptasensing comparison}

As presented in Figure 7 , the 96 nt and 80 nt aptamers $[148,149]$ displayed a more complex structure, mostly expressed by the formation of multiple stem loops, in contrast with the simple folded organization of the reduced aptamers and minimers $[150,167]$. The final structure, predicted in Mfold, relied on the folding temperature, commonly varying from ice to room temperature, along with the ions present in the buffer $\left(\mathrm{Mg}^{+2}, \mathrm{Na}^{+}\right)$. On that note, ongoing attempts by our research group to develop a AuNP-based colorimetric assay unveiled the role of different binding buffers on the final assay specificity [200]. Unlike previous aptasensors [164, 167], assays with the $40 \mathrm{nt}$ aptamer under the presence of Tris $\mathrm{HCl}$ denoted lack of specificity when OTA was included [200].
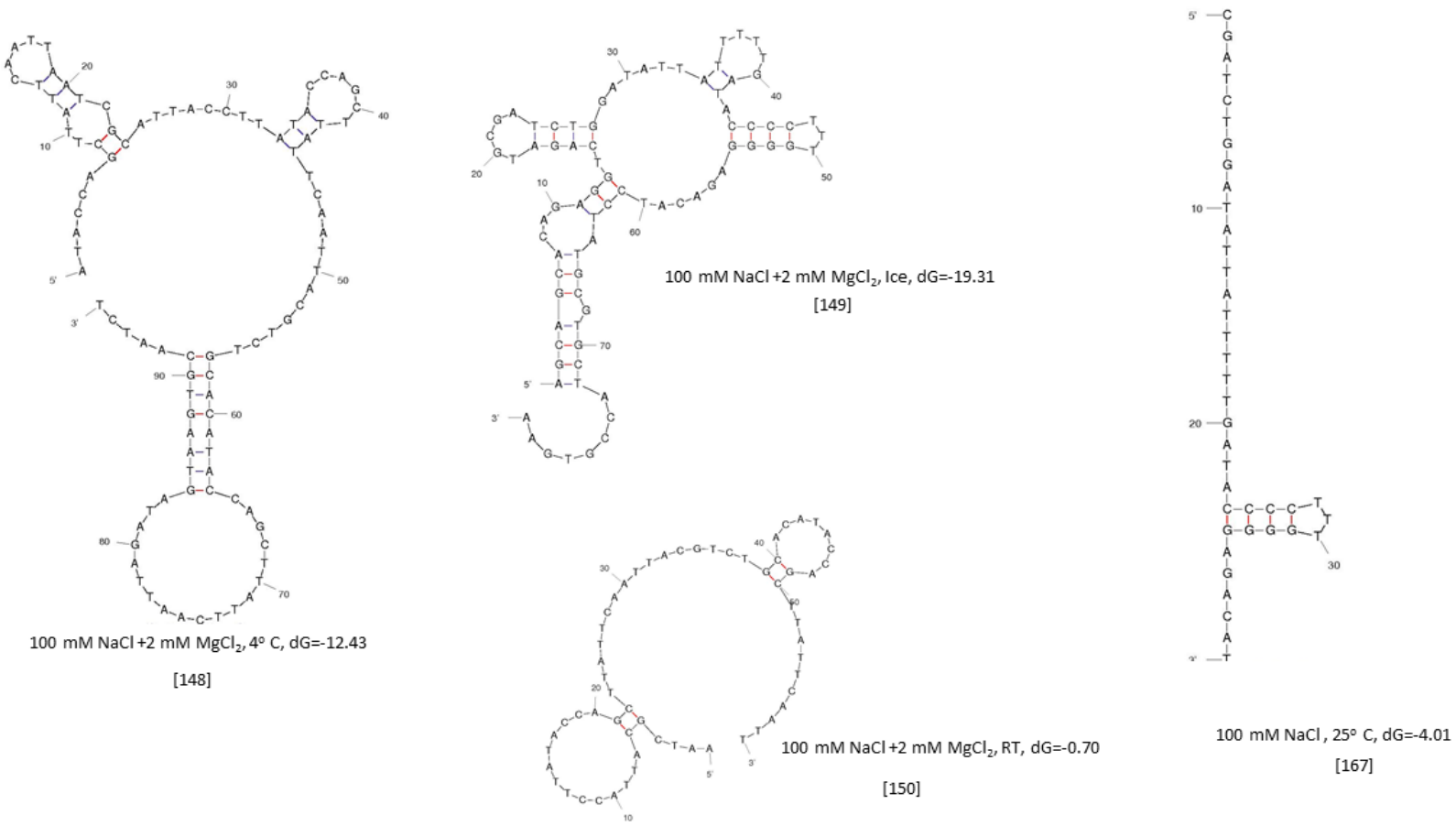

Figure 7. Aptamer folding forms obtained in Mfold at the specified conditions

A PCA specific to all the aptamer-based biosensors for FB1, is indicated in Figure 8, by using LODmax, ATmax and APmax of $100 \mu \mathrm{g} / \mathrm{L}$ [169], 720 minutes [166] and 12900 minutes [151], respectively. As already noted, assays with a hybridized $96 \mathrm{nt}$ aptamer were mainly correlated to the lowest LOD's through fluorescent [151, 152, 161, 166, 170], chemiluminescent [155], optical [169] and MS [197] detections, along with fluorescent and SERS signals obtained from a non-specified hybridized aptamer $[177,198]$. On the other hand, the shortest assay times were correlated to applications with the $96 \mathrm{nt}$ aptamer in its end-modified [157, 160,163, 168] and hybridized forms [158, $165,173,174]$, as well as electrochemical designs with some shorter sequences including a thiol modified $80 \mathrm{nt}$ aptamer[156] and an unmodified $40 \mathrm{nt}$ [164] sequence. Likewise, the assay preparation time showed high correlation to $60 \mathrm{nt}$ fluorescent [159], $40 \mathrm{nt}$ electrochemical [167], and $96 \mathrm{nt}$ 
colorimetric [200] aptasensors. Nevertheless, as already stated, the high correlation of the $96 \mathrm{nt}$ aptamer with a high sensitivity (low LODs) in combination with its convenient specificity, were relevant for the existence of more biosensors based on this long length sequence. In addition, more robust techniques might be ideal for increasing the sensitivity of aptasensors. For instance, the analysis of the signals from the unique complex produced by the incubation of the $96 \mathrm{nt}$ aptamer, FB1 and AuNP in particular buffer conditions $\left(\mathrm{MgCl}_{2} 1 \mathrm{mM}\right)$, can be translated to LODs as low as $3 \mu \mathrm{g} / \mathrm{L}$ levels (analysis of spectral scan) with a refining to $56 \mathrm{pg} / \mathrm{L}$, when employing techniques such as asymmetric flow field-flow fractionation (AF4) for resolving those complexes [200]. It is worth noting that no paperbased biosensor has so far been developed with any of the aptamers, whose application could reduce the cost and extend the applicability of such sensitive conformations.

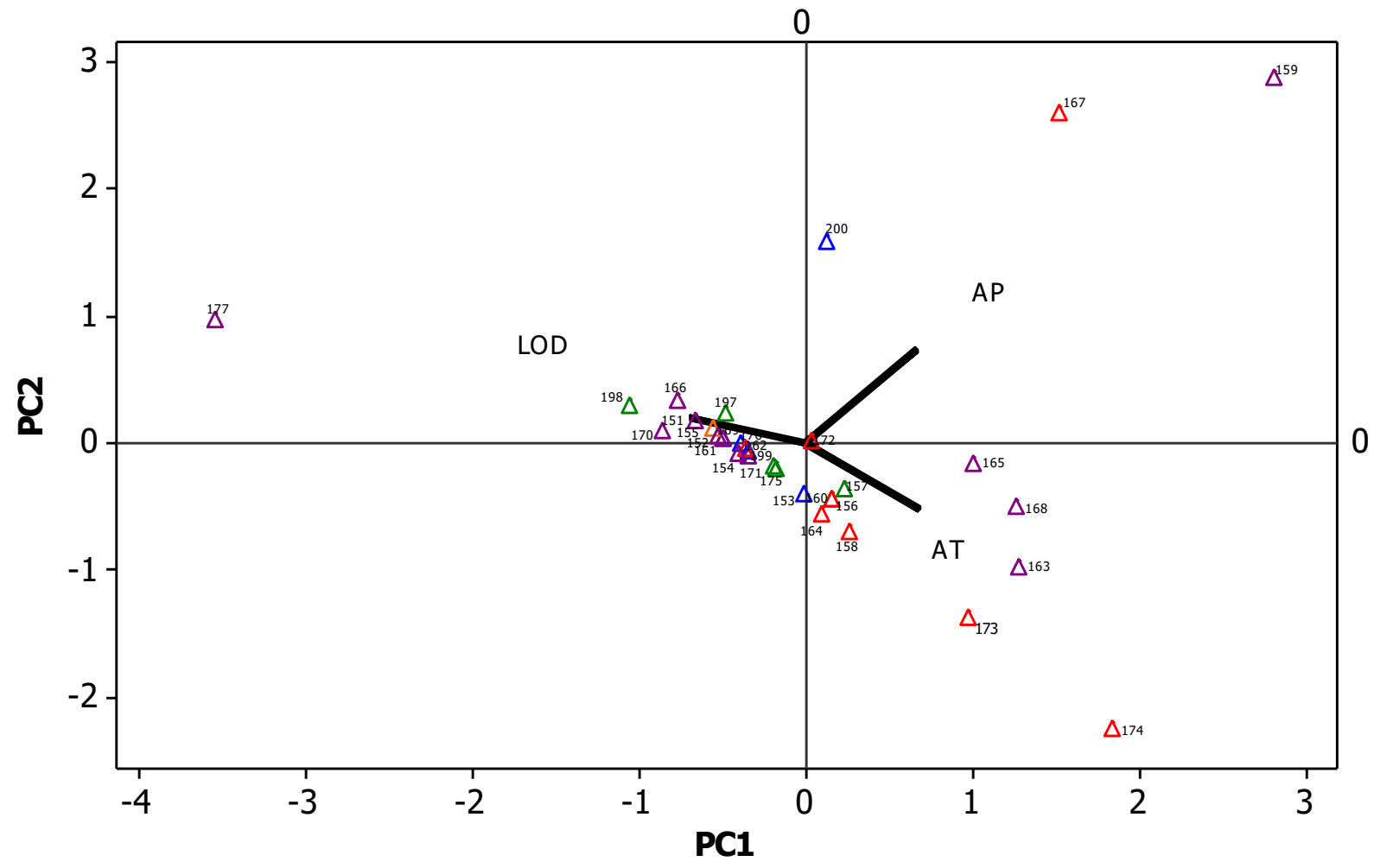

Figure 8. Principle component analysis for the correlation of all the reported aptasensors for optical $(\Delta)$, fluorescent $(\Delta)$, chemiluminescent $(\Delta)$, electrochemical $(\Delta)$, and other signals $(\Delta)$. The numbers correspond to the correlated references from Table 5.

\section{Conclusions}

The use of aptamers for the quantification of fumonisin B1 is at the central focus in the field of biosensors with many areas of opportunity, on account of their relatively recent dissemination and the few strands already reported. Even when sensitive, aptasensors featured similar or lower detections limits than well-established immunosensing techniques, LC-MS assays and Raman-based methods, the recent application of MIPS has redirected the attention on the improvement of the LODs from aptamer-based biosensors. Additionally, despite the diversity of approaches performed with the two selected aptamers and their shortened forms, to date around $95 \%$ of all the aptasensors have been proposed as bulk experiments. Hence, there is considerable room of opportunity for the exploration of different supports, ideally paper matrices for the refinement of on-site testing. Additionally, reducing the extraction steps is a desirable quality for quick analysis of samples in remote areas.

Thus far, the specificity of the aptamers utilized for FB1 quantification has been confirmed against up to 19 different molecules, and in multiplex detections of up to 4 targets, while their limits of detection confirmed the feasibility of addressing contamination levels under the regulated limits. It is important to understand and uncover the role of the selected support, and binding conditions (binding buffer, temperature, time) on the selectivity and affinity of the resulting biosensor. Despite all 
the advances regarding aptamers, more efforts are necessary to obtain shorter strands with high affinity towards FB1 or novel targets, so the final sensing method can be simplified, yet be effective.

Acknowledgments: V.A. Miron-Merida acknowledges Mexico's National Council of Science and Technology (CONACyT) for their support in his Postgraduate Studies through and Academic Scholarship.

Conflicts of Interest: The authors declare no conflict of interest.

\section{References}

1. Marin, S., Ramos, A.J.; Cano-Sancho, G.; Sanchis, V. Mycotoxins: Occurrence, toxicology, and exposure assessment. Food Chem Toxicol, 2013, 60, pp.218-237.

2. Karlovsky, P.; Shman, M.; Berthiller, F.; De Meester, J.; Eisenbrand, G.; Perrin, I.; Oswald, I.P.; Speijers, G.; Chiodini, A.; Recker, T.; Dussort, P. Impact of food processing and detoxification treatments on mycotoxin contamination. Mycotoxin Res 2016, 32(4), pp.179-205.

3. Bennett, J.W.; Klich, M. Mycotoxins. Clin Microbiol Rev 2003, 16(3), pp.497-516.

4. European Commission. 2018. RASFF portal. Available online: https://ec.europa.eu/food/safety/rasff en (Accessed on 07 April 2020)

5. Lamprecht, S.C.; Marasas, W.F.O.; Alberts, J.F.; Cawood, M.E.; Gelderblom, W.C.A.; Shephard, G.S.; Thiel, P.G.; Calitz, F.J. Phytotoxicity of fumonisins and TA-toxin to corn and tomato. Phytopathology 1994, 84(4), pp.383-391.

6. Rheeder, J.P.; Marasas, W.F.O.; Vismer, H.F. Production of fumonisin analogs by Fusarium species. Appl Environ Microbiol 2002, 68(5), pp.2101-2105.

7. Gelderblom, W.C.A.; Jaskiewicz, K.; Marasas, W.F.O.; Thiel, P.G.; Horak, R.M.; Vleggar, R.; Kriek, N.P.J. Fumonisins- Novel mycotoxins with cancer-promoting activity produced by Fusarium moniliforme. Appl Environ Microbiol 1988, 54(7), pp.1806-1811.

8. Abbas, H.K.; Vesonder, R.F.; Boyette, C.D.; Hoagland, R.E.; Krick, T. Production of fumonisins by Fusarium moniliforme cultures isolated from jimsonweed in Mississippi. J Phytopathol 1992, 136(3), pp.199-203.

9. Bezuidenhout, S.C.; Gelderblom, W.C.A.; Gorst-Allman, C.P.; Horak, R.M.; Marasas, W.F.O.; Spiteller, G.; Vleggaar, R. Structure elucidation of the fumonisins, mycotoxins from Fusarium moniliforme. $J$ Chem Soc D 1988, O(11), pp.743-745.

10. Branham, B.E.; Plattner, R.D. Isolation and characterization of a new fumonisin from liquid cultures of Fusarium moniliforme. J Nat Prod 1993, 56(9), pp.1630-1633.

11. Abbas, H.K.; Riley, R.T. The presence and phytotoxicity of fumonisins and AAL-toxin in Alternaria alternate. Toxicon 1995, 34(1), pp.133-136.

12. Frisvad, J.C.; Smedsgaard, J.; Samson, R.A.; Larsen, T.O.; Thrane, U. Fumonisin B 2 production by Aspergillus niger. J Agric Food Chem 2007, 55(23), pp.9727-9732.

13. Månsson, M.; Klejnstrup, M.L.; Phipps, R.K.; Nielsen, K.F.; Frisvad, J.C.; Gotfredsen, C.H.; Larsen, T.O. Isolation and NMR characterization of Fumonisin $\mathrm{B}_{2}$ and a new fumonisin $\mathrm{B}_{6}$ from Aspergillus niger. J Agric Food Chem 2010, 58(2), pp.949-953.

14. Mogensen, J.M.; Møller, K.A.; Freiesleben, P.; Labuda, R.; Varga, E.; Sulyok, M.; Kubatova, A.; Thrane, U.; Andersen, B.; Nielsen, K.F. Production of fumonisins $B_{2}$ and $B_{4}$ in Tolypocladium species. $J$ Ind Microbiol Biotechnol 2011, 38(9), pp.1329-1335.

15. Ostry, V.; Malir, F.; Toman, J.; Grosse, Y. Mycotoxins as human carcinogens-the IARC Monographs classification. Mycotoxin Res 2017, 33(1), pp.65-73. 
16. Gutleb, A.C.; Morrison, E.; Murk, A.J. Cytotoxicity assays for mycotoxins produced by Fusarium strains: a review. Environ Toxicol Pharmacol 2002, 11(3-4), pp.309-320.

17. Riley, R.T.; Merrill, A.H. Ceramide synthase inhibition by fumonisins: a perfect storm of perturbed sphingolipid metabolism, signaling, and disease. J Lipid Res 2019, 60(7), pp.1183-1189.

18. Régnier, M.; Polizzi, A.; Lukowicz, C.; Smati, S.; Lasserre, F.; Lippi, Y.; Naylies, C.; Laffitte. J.; Bétoulières, C.; Montagner, A.; Ducheix, S.; Gourbeyre, P.; Ellero-Simatos, S.; Menard, S.; Bertrand-Michel, J.; Al Saati, T.; Lobaccaro, J.M.; Burger, H.M.; Gelderblom, W.C.; Guillou, H.; Oswald, I.P.; Loiseau, N. The protective role of liver $X$ receptor (LXR) during fumonisin B1-induced hepatotoxicity. Arch Toxicol 2019, 93, pp. 505-517.

19. Liu, X.; Fan, L.; Yin, S.; Chen, H.; Hu, H. Molecular mechanisms of fumonisin B1-induced toxicities and its applications in the mechanism-based interventions. Toxicon 2019, 167, pp.1-5.

20. Yuan, Q.; Jiang, Y.; Fan, Y.; Ma, Y.; Lei, H.; Su, J. Fumonisin B1 induces oxidative stress and breaks barrier functions in pig iliac endothelium cells. Toxins 2019, 11, pp.387.

21. Sharma, S.K.; Sharma, S.P.; Miller, D.; Parel. J.M.A.; Leblanc, R.M. Interfacial behavior of fumonisin B1 toxin and its degradation on the membrane. Langmuir 2019, 35(7), pp.2814-2820.

22. Marasas, W.F.O.; Jaskiewicz, K.; Venter, F.S.; Van Schalkwyk, D.J. Fusarium moniliforme contamination of maize in oesophageal cancer areas in Transkei. S Afr Med J 1988, 74(3), pp.110-114.

23. Yoshizawa, T.; Yamashita, A.; Luo, Y. Fumonisin occurrence in corn from high- and low-risk areas for human esophageal cancer in China. Appl Environ Microbiol 1994, 60(5), pp.1626-1629.

24. Missmer, S.A.; Suarez, L.; Felkner, M.; Wang, E.; Merrll Jr.; A. H.; Rothman, K.J.; Hendricks, K.A. Exposure to fumonisins and the occurrence of neural tube defects along the Texas-Mexico border. Environ Health Perspect 2006, 114(2), pp.237-241.

25. Magoha, H.; De Meulenaer, B.; Kimanya, M.; Hipolite, C.L.; Kolsteren, P. Fumonisin B1 contamination in breast milk and its exposure in infants under 6 months of age in Rombo, Nothern Tanzania. Food Chem Toxicol 2014, 74, 112-116.

26. Riley, R.T.; Torres, O.; Matute. J.; Gregory, S.G.; Ashley-Koch, A.E.; Showker, J.L.; Mitchell, T.; Voss, K.A.; Maddox, J.R.; Gelineau-van Waes, J.B. Evidence for fumonisin inhibition of ceramide synthase in humans consuming maize-based foods and living in high exposure communities in Guatemala. Mol Nutr Food Res 2015, 59(11), pp.2209-2224.

27. Torres, O.; Matute, J.; Gelineau-van Waes, J.; Maddox, J.R.; Gregory, S.G.; Ashley-Koch, A.; Showker, J.I.; Zitomer, N.C.; Voss, K.A.; Riley, R.T. Urinary fumonisin B1 and estimated fumonisin intake in women from high- and low- exposure communities in Guatemala. Mol Nutr Food Res 2014, 58(5), pp.973-983.

28. Chen, C.; Mitchell, N.J.; Gratz, J.; Houpt, E.R.; Gong, Y.; Egner, P.A.; Groopman, J.D.; Riley, R.T.; Showker, J.L.; Svensen, E.; Mduma, E.R.; Patil, C.L.; Wu, F. Exposure to aflatoxin and fumonisin in children at risk for growth impairment in rural Tanzania. Environ Int 2018, 115, pp.29-37.

29. Food and Agriculture Organization of the United Nations. 2004. Worldwide regulations for mycotoxins in food and feed in 2003 (31 December 2003). Available online: http://www.fao.org/docrep/007/y5499e/y5499e00.htm (Accessed on 31 October 2017)

30. Udomkun, P.; Wiredu, A.N.; Nagle, M.; Bandyopadhyay, R.; Müller, J.; Vanlauwe, B. Mycotoxins in SubSaharan Africa: Present situation, socio-economic impact, awareness, and outlook. Food Contro/2017, 72(A), pp.110-122. 
31. Bartók, T.; Szécsi, A.; Szekeres, A.; Mesterházy, A.; Bartók, M. Detection of new fumonisin mycotoxins and fumonisin-like compounds by reversed-phase high-performance liquid chromatography/electrospray ionization ion trap mass spectrometry. Rapid Commun Mass Spectrom 2006, 20(16), pp.2447-2462.

32. Savi, G.D.; Piacentini, K.C.; Marchi, D.; Scussel, V.M. Fumonisins $\mathrm{B}_{1}$ and $\mathrm{B}_{2}$ in the corn-milling process and corn-based products, and evaluation of estimated daily intake. Food Addit Contam: Part A 2016, 33(2), pp.339-345.

33. Yamagishi, D.; Akamatsu, H.; Otani, H.; Kodama, M. Pathological evaluation of host-specific AAL-toxins and fumonisin mycotoxins produced by Alternaria and Fusarium species. J Gen Plant Pathol 2006, 72(5), pp.323-327.

34. Noonim, P.; Mahakarnchanakul, W.; Nielsen, K.F.; Frisvad, J.C.; Samson, R.A. Fumonisin B 2 production by Aspergillus niger in Thai coffee beans. Food Addit Contam: Part A 2009, 26(1), pp.94100.

35. Varga, J.; Kocsubé, S.; Suri, K.; Szigeti, G.; Szekeres, A.; Varga, M.; Tóth, B.; Bartók, T. Fumonisin contamination and fumonisin producing black Aspergilli in dried vine fruits of different origin. Int $\mathrm{J}$ Food Microbiol 2010, 143(3), pp.143-149.

36. Hossain, S.M.; Luckham, R.E.; Smith, A.M.; Lebert, J.M.; Davies, L.M.; Pelton, R.H.; Filipe, C.; Brennan, J.D. Development of a bioactive paper sensor for detection of neurotoxins using piezoelectric inkjet printing of sol-gel-derived bioinks. Anal Chem 2009, 81(13), pp.5474-5483.

37. Lee, S.; Kim, G.; Moon, J. Performance improvement of the one-dot lateral flow immunoassay for Aflatoxin B1 by using a smartphone-based reading system. Sensors 2013, 13(4), pp.5109-5116.

38. Ueno, Y.; lijima, K.; Wang, S.-D.; Sugiura, Y.; Sekijima, M.; Tanaka, T.; Chen, C.; Yu, S.-Z. Fumonisins as a possible contributory risk factor for primary liver cancer: a 3-year study of corn harvested in Haimen, China, by HPLC and ELISA. Food Chem Toxicol 1997, 35(12), pp.1143-1150.

39. Campa, R.; Miller, D.; Hendricks, K. Fumonisin in tortillas produced in small-scale facilities and effect of traditional masa production methods on this mycotoxin. J Agric Food Chem 2004, 52, pp.4432-4437.

40. Gong, Y.Y.; Torres-Sanchez, L.; Lopez-Carrillo, L.; He Peng, J.; Sutcliffe, A.E.; White, K.L.; Humpf, HU.; Turner, P.C.; Wild, C.P. Association between tortilla consumption and human urinary fumonisin B1 levels in a Mexican population. Cancer Epidemiol Biomarkers Prev, 2008, 17(3), pp.688-694.

41. Dall'Asta, C.; Mangia, M.; Berthiller, F.; Molinelli, A.; Sulyok, M.; Schuhmacher, R.; Krska, R.; Galaverna, G.; Dossena, A.; Marchello, R. Difficulties in fumonisin determination: the issue of hidden fumonisins. Anal BioanalChem 2009, 395(5), pp.1335-1345.

42. Ghali, R.; Ghorbel, H.; Hedilli, A. Fumonisin determination in Tunisian foods and feeds. ELISA and HPLC methods comparison. J Agric Food Chem 2009, 57(9), pp.3955-3960.

43. Gazzotti, T.; Lugoboni, B.; Zironi, E.; Barbarossa, A.; Serraino, A.; Pagliuca, G. Determination of fumonisin B1 in bovine milk by LC-MS/MS. Food Control 2009, 20(12), pp.1171-1174.

44. Silva, L.J.G.; Pena, A.; Lino, C.M.; Fernández, M.F.; Mañes, J. Fumonisin determination in urine by LCMS-MS. Anal BioanalChem 2010, 396(2), pp.809-816.

45. Tansakul, N.; Jala, P.; Laopiem, S.; Tangmunkhong, P.; Limsuwan, S. Co-occurrence of five Fusarium toxins in corn-dried distiller's grains with solubles in Thailand and comparison of ELISA and LC-MS/MS for fumonisin analysis. Mycotoxin Res 2013, 29(4), pp.255.260.

46. Petrarca, M.H.; Rodrigues, M.I.; Rossi, E.A.; De Sylos, C.M. Optimisation of a simple preparation method for the determinations of fumonisin B1 in rice. Food Chem 2014, 158, pp.270-277. 
47. Bordin, K.; Rottinghaus, G.E.; Landers, B.R.; Ledoux, D.R.; Kobashigawa, E.; Corassin, C.H.; Oliveira, C.A.F. Evaluation of fumonisin exposure by determination of fumonisin B1 in human hair and in Brazilian corn products. Food Control 2015, 53, pp.67-71.

48. Liu, H.; Luo, J.; Kong, W.; Liu, Q.; Hu, Y.; Yang, M. UFLC-ESI-MS/MS analysis of multiple mycotoxins in medicinal and edible Areca catechu. Chemosphere 2016, 150, pp.176-183.

49. Li, M.; Kong, W.; Li, Y.; Liu, H.; Liu, Q.; Dou, X.; Ou-yang, Z.; Yang, M. High-throughput determination of multi-mycotoxins in Chinese yam and related products by ultra fast liquid chromatography coupled with tandem mass spectrometry after one-step extraction. J Chromatogr B 2016, 1022, pp. 118-125.

50. Xing, Y.; Meng, W.; Sun, W.; Li, D.; Yu, Z.; Tong, L.; Zhao, Y. Simultaneous qualitative and quantitative analysis of 21 mycotoxins in Radix Paeoniae Alba by ultra-high performance liquid chromatography quadrupole linear ion trap mass spectrometry and QuEChERS for sample preparation. J Chromatogr B 2016, 1031, pp.202-213.

51. Danezis, G.P.; Anagnostopoulos, C.J.; Liapis, K.; Koupparis, M.A. Multi-residue analysis of pesticides, plant hormones, veterinary drugs and mycotoxins using HILIC chromatography e MS/MS in various food matrices. Anal Chim Acta 2016, 942, pp. 121-138.

52. Zhang, S.; Lu, J.; Wang, S.; Mao, D.; Miao, S.; Ji, S. Multy-mycotoxins analysis in Pheretima using ultra-high-performance liquid chromatography tandem mass spectrometry based on a modified QuEChERS method. J Chromatogr B 2016, 1035, pp.31-41.

53. Dagnac, T.; Latorre, A.; Fernández, B.; Maria, M. Validation and application of a liquid chromatographytandem mass spectrometry based method for the assessment of the cooccurrence of mycotoxins in maize silages from dairy farms in NW Spain. Food Addit Contam: Part A 2016, 33(12), pp.1850-1863.

54. Sun, J.; Li, W.; Zhang, Y.; Hu, X.; Wu, L.; Wang, B. QuEChERS purification combined with ultrahighperformance liquid chromatography tandem mass spectrometry for simultaneous quantification of 25 mycotoxins in cereals. Toxins 2016, 8, pp.375-392.

55. Smith, L.L.; Francis, K.A.; Johnson, J.T.; Gaskill, C.L. Quantification of fumonisin B1 and B2 in feed using FMOC pre-column derivatization with HPLC and fluorescence detection. Food Chem 2017, 234, pp.174-179.

56. Souto, P.C.M.C.; Jager, A.V.; Tonin, F.G.; Petta, T.; Di Gregório, M.C.; Cossalter, A-M.; Pinton, P.; Oswald, I.P.; Rottinghaus, G.E.; Oliveira, A.A.F. Determination of fumonisin B1 levels in body fluids and hair from piglets fed fumonisin B1-contaminates diets. Food Chem Toxicol 2017, 108(A), pp.1-9.

57. Osteresch, B.; Viegas, S.; Cramer, B.; Humpf, H-U. Multi-mycotoxin analysis using dried blood spots and dried serum spots. Anal BioanalChem 2017, 409, pp.3369-3382.

58. Flores-Flores, M.E.; González-Peñas, E. An LC-MS/MS method for multi-mycotoxin quantification in cow milk. Food Chem 2017, 218, pp.378-385.

59. Zhao, Y.; Wan, L.; Bai, X.; Liu, Y.; Zhang, F.; Liu, Y.; Liao, X. Quantification of mycotoxins in vegetable oil by UPLC-MS/MS after magnetic solid-phase extraction. Food Addit Contam: Part A 2017, 34(7),pp.1201-1210.

60. Annunziata, L.; Stramenga, A.; Visciano, P.; Schirone, M.; De Colli, L.; Novella, M.; Campana, G.; Scortichini, G. Simultaneous determination of aflatoxins, T-2 and HT-2 toxins, and fumonisins in cerealderived products by QuEChERS extraction coupled with LC-MS/MS. Anal BioanalChem 2017, 409, pp.5143-5155. 
61. Miró-Abella, E.; Herrero, P.; Canela, N.; Arola, L.; Borrull, F.; Ras, R.; Fontanals, N. Determination of mycotoxins in plant-based beverages using QuEChERS and liquid chromatography-tandem mass spectrometry. Food Chem 2017, 229, pp.366-372.

62. Abia, W.A.; Warth, B.; Ezekiel, C.N.; Sarkanj, B.; Turner, P.C.; Marko, D.; Krska, R.; Sulyok, M. Uncommon toxic microbial metabolite patterns in traditionally home-processed maize dish (fufu) consumed in rural Cameroon. Food Chem Toxicol 2017, 107, pp.10-19.

63. Hamed, A.M.; Arroyo-Manzanares, N.; García-Campaña, A.M.; Gámiz-Gracia, L. Determination of Fusarium toxins in functional vegetable milks applying salting-out-assisted liquid-liquid extraction combined with ultra-high performance liquid chromatography tandem mass spectrometry. Food Addit Contam: Part A 2017, 34(11), pp.2033-2041.

64. Zhao, X.S.; Kong, W.J.; Wang, S.; Wei, J.H.; Yang, M.H. Simultaneous analysis of multiple mycotoxins in Alpinia oxyphylla by UPLC-MS/MS. World Mycotoxin Journal 2017, 10(1), pp.41-51.

65. Du, L.-J.; Chu, C.; Warner, E.; Wang, Q.-Y.; Hu, Y.-H.; Chai, K.-J.; Cao, J.; Peng, L.-Q.; Chen, Y.-B.; Yang, J.; Zhang, Q.-D. Rapid microwave-assisted dispersive micro-solid phase extraction of mycotoxins in food using zirconia nanoparticles. J Chromatogr A 2018,1561, pp.1-12.

66. Huang, P.; Kong, W.; Wang, S.; Wang, R.; Lu, J.; Yang, M. Multiclass mycotoxins in lotus seeds analysed by an isotope-labelled internal standard-based UPLC-MS/MS. J Pharm Pharmacol 2018, 70, pp.1378-1388.

67. Zhang, B.; Chen, X.; Han, S.-Y.; Li, M.; Ma, T.-Z.; Sheng, W.-J.; Zhu, X. Simultaneous analysis of 20 mycotoxins in grapes and wines from Hexi corridor region (China): based on a QuEChERS-UHPLCMS/MS method. Molecules 2018, 23(8), 1926.

68. Abdallah, M.F.; Krska, R.; Sulyok, M. Occurrence of ochratoxins, fumonisin B2, aflatoxins(B1 and B2), and other secondary fungal metabolites in dried date palm fruits from Egypt: A mini-survey. $J$ Food Sci 2018, 83(2), pp.559-564.

69. De Baere, S.; Croubels, S.; Novak, B.; Bichl, G.; Antonissen, G. Development and validation of a UPLCMS/MS and UPLC-HR-MS method for the determination of fumonisin B1 and its hydrolysed metabolites and fumonisin B2 in broiler chicken plasma. Toxins 2018, 10(2), 62.

70. Cladière, M.; Delaporte, G.; Le Roux, E.; Came, V. Multi-class analysis for simultaneous determination of pesticides, mycotoxins, process-induced toxicants and packaging contaminants in tea. Food Chem 2018, 242, pp.113-121.

71. Carballo, C.; Font, G.; Ferrer, E.; Berrada, H. Evaluation of mycotoxin residues on ready-to-eat food by chromatographic methods coupled to mass spectrometry in tandem. Toxin 2018, 10(10), 243.

72. Park, J.; Kim, D.-H.; Moon, J.-Y.; An, J.-A.; Kim, Y.-W.; Chung, S.-H.; Lee, C. Distribution analysis of twelve mycotoxins in corn and corn-derived products by LC-MS/MS to evaluate the carry-over ratio during wet-milling. Toxins 2018, 10(8), 319.

73. Šarkanj, B.; Ezekiel, C.N.; Turner, P.C.; Abia, W.A.; Rychlik, M.; Krska, R.; Sulyok, M.; Warth, B. Ultrasensitive, stable isotope assisted quantification of multiple urinary mycotoxin exposure biomarkers. Anal Chim Acta 2018, 1019, pp.84-92.

74. González-Jartín, J. M; Alfonso, A.; Rodríguez, I.; Sainz, M. J.; Vieytes, M. R.; Botana, L. M. A. QuEChERS based extraction procedure coupled to UPLC-MS/MS detection for mycotoxins analysis in beer. Food Chem 2019, 275, pp.703-710. 
75. Da Silva, L.P.; Madureira, F.; De Azevedo, E.; Ferreira, A.; Augusti, R. Development and validation of a multianalyte method for quantification of mycotoxins and pesticides in rice using a simple dilute and shoot procedure and UHPLC-MS/MS. Food Chem 2019, 270, pp.420-427.

76. Bessaire, T.; Perrin, I.; Tarres, A.; Bebius, A.; Reding, F.; Theurillat, V. Mycotoxins in green coffee: Occurrence and risk assessment. Food Control 2019, 96, pp.59-67.

77. Jedziniak, P.; Panasiuk, L.; Pietruszka, K.; Posyniak, A. Multiple mycotoxins analysis in animal feed with LC-MS/MS: Comparison of extract dilution and immune affinity clean-up. J Sep Sci 2019, 42, pp.1240-1247.

78. Nafuka, S. N.; Misihairabgwi, J. M.; Bock, R.; Ishola, A.; Sulyok, M.; Krska, R. Variation of fungal metabolites in sorghum malts used to prepare Namibian traditional fermented beverages Omalodu and Otombo. Toxins 2019, 11(3), pp.165.

79. Abdallah, M. F.; Audenaert, K.; Lust, L.; Landschoot, S.; Bekaert, B.; Haesaert, G.; De Boevre, M.; De Saeger, S. Risk characterization and quantification of mycotoxins and their producing fungi in sugarcane juice: A neglected problem in a widely-consumed traditional beverage. Food Control 2020, 108, pp.106811.

80. Hort, V.; Nicolas, M.; Travel, A.; Jondreville, C.; Maleix, C.; Baéza, E.; Engel, E.; Guérin, T. Carry-over assessment of fumonisins and zearalenone to poultry tissues after exposure of chickens to a contaminated diet-A study implementing stable-isotope dilution assay and UHPLC-MS/MS. Food Control 2020, 107, pp.106789.

81. Thompson, V.S.; Maragos, C.M. Fiber-optic immunosensor for the detection of fumonisin B1. J Agric Food Chem 1996, 44(4), pp.1041-1046.

82. Mullett, W.; Lai, E.P.C.; Yeung, J.M. Immunoassay of fumonisins by a surface plasmon resonance biosensor. Anal Biochem 1998, 258(2), pp.161-167.

83. Ho, J.A.; Durst, R.A. Development of a flow-injection liposome immunoanalysis system for fumonisin B1. Anal Chim Acta 2000, 414(1-2), pp.61-69.

84. Maragos, C.M.; Jolley, M.E.; Plattner, R.D.; Nasir, M.S. Fluorescence polarization as means for determination of fumonisin in maize. Journal of Agricultural and Food Chem 2011, 49(2), pp.596-602.

85. Ligler, F.S.; Taitt, C.R.; Shriver-Lake, L.C.; Sapsford, K.E.; Shubin, Y.; Golden, J.P. Array biosensor for detection of toxins. Anal BioanalChem 2003, 377(3), pp.469-477.

86. Quan, Y.; Zhang, Y.; Wang, S.; Lee, N.; Kennedy, I.R. A rapid and sensitive chemiluminescence enzyme-linked immunosorbent assay for the determinations of fumonisins B1 in food samples. Anal Chim Acta 2006, 580(1), pp.1-8.

87. Lamberti, I.; Tanzarella, C.; Solinas, I.; Padula, C.; Mosiello, L. An antibody-based microarray assay for the simultaneous detection of aflatoxin B1 and fumonisin B1. Mycotoxin Res 2009, 25, pp.193-200.

88. Molinelli, A.; Grossalber, K.; Krska, R. A rapid lateral flow test for the determination of total type B fumonisins in maize. Anal BioanalChem 2009, 395, pp.1309-1316.

89. Anderson, G.P.; Kowtha, V.A.; Taitt, T.C. Detection of fumonisin B1 and Ochratoxin A in grain products using microsphere- based fluid array immunoassays. Toxins 2010, 2(2), pp.297-309.

90. Kadir, M.K.; Tothill, I.E. Development of an electrochemical immunosensor for fumonisins detection in foods. Toxins 2010, 2(4), pp.382-398.

91. Anfossi, L.; Calderara, M.; Baggiani, C.; Giovannoli, C.; Arletti, E.; Giraudi, G. Development and application of a quantitative lateral flow immunoassay for fumonisins in maize. Anal Chim Acta 2010, 682(1-2), pp.104-109. 
92. Wang, X.; Zhang, H.; Liu, H.; He, C.; Zhang, A.; Ma, J.; Ma, Y.; Wu, W. and Zheng, H. An immunoarray for the simultaneous detection of two mycotoxins, ochratoxin A and fumonisin B1. J Food Saf 2011, 31(3), pp.408-416.

93. Mirasoli, M.; Buragina, A.; Dolci, L.S.; Simoni, P.; Anfossi, L.; Giraudi, G.; Roda, A. Chemiluminescencebased biosensor for fumonisins quantitative detection in maize samples. Biosens Bioelectron 2012 , 32(1), pp.283-287.

94. Li, Y.-S.; Zhou, Y.; Lu, S.-Y.; Guo, D.-J.; Ren, H.-L.; Meng, X.-M.; Zhi, B.-H.; Lin, C.; Wang, Z.; Li, X.B.; Liu, Z.-S. Development of a one-step strip for rapid screening of fumonisins $B_{1}, B_{2}$ and $B_{3}$ in maize. Food Control 2012, 24(1-2), pp.72-77.

95. Lattanzio, V.M.T.; Nirvarlet, N.; Lippolis, V.; Gatta, S.D.; Huet, A.-C.; Delahaut, P.; Granier, B.; Visconti, A. Multiplex dipstick immunoassay for semi-quantitative determination of Fusarium mycotoxins in cereals. Anal Chim Acta 2012, 718, pp.99-108.

96. Zou, L.; Xu, Y.; Li, Y.; He, Q.; Chen, B.; Wang, D. Development of a single-chain variable fragment antibody-based enzyme-linked immunosorbent assay for determination of fumonisin B1 in corn samples. J Sci Food Agric 2013, 94(9), pp.1865-1871.

97. Peters, J.; Thomas, D.; Boers, E.; Rijk, T.; Berthiller, F.; Hasnoot, W.; Nielen, M.W.F. Colour-encoded paramagnetic microbead-based direct inhibition triplex flow cytometric immunoassay for ochratoxin $A$, fumonisins and zearalenone in cereals and cereal-based feed. Anal BioanalChem 2013, 405(24), pp.7783-7794.

98. Wang, Y-K.; Yan, Y-X.; Ji, W-H.; Wang, H.; Li, S-Q.; Zou, Q.; Sun, J-H. Rapid simultaneous quantification of zearalenone and fumonisin $B_{1}$ in corn and wheat by lateral flow dual immunoassay. $J$ Agric Food Chem 2013, 61(21), pp.5031-5036.

99. Venkataramana, M.; Navya, K.; Chandranyaka, S.; Privanka, S.R.; Murali, H.S.; Batra, H.V. Development and validation of an immunochromatographic assay for rapid detection of fumonisin B1 from cereal samples. J Food Sci Technol 2014, 51(9), pp.1920-1928.

100.Ezquerra, A.; Vidal, J.C.; Bonel, L.; Castillo, J.R. A validated multi-channel electrochemical immunoassay for rapid fumonisin B1 determination in cereal samples. Anal Methods 2015, 7, pp.37423749.

101.Masikini, M.; Mailu, S.N.; Tsegaye, A.; Njomo, N.; Molapo, K.M.; Ikpo, C.O.; Sunday, C.E.; Rassie, C.; Wilson, L.; Baker, P.G.L.; Iwuoha, E.I. A fumonisin immunosensor based on polyanilino-carbon nanotubes doped with palladium telluride quantum dots. Sensors 2015, 15(1), pp.529-546.

102.Zangheri, M.; Nardo, F.; Anfossi, L.; Giovannoli, C.; Baggiani, C.; Roda, A.; Mirasoli, M. A multiplex chemiluminescent biosensor for type B-fumonisins and aflatoxin B1 quantitative detection in maize flour. Analyst 2015, 140, pp.358-365.

103.Yang, X.; Zhou, X.; Zhang, X.; Qing, Y.; Luo, M.; Liu, X.; Li, C.; Li, Y.; Xia, H.; Qiu, J. A highly sensitive electrochemical immunosensor for fumonisin B1 detection in corn using single-walled carbon nanotubes/chitosan. Electroanalysis 2015, 27(11), pp.2679-2687.

104.Jodra, A.; López, M.A.; Escarpa, A. Disposable and reliable electrochemical magnetoimmunosensor for fumonisins simplified determinations in maize-based foodstuffs. Biosens Bioelectron 2015, 64, pp.633-638.

105.Shu, M.; Xu, Y.; Wang, D.; Liu, X.; Li, Y.; He, Q.; Tu, Z.; Qiu, Y.; Ji, Y.; Wang, X. Anti-idiotypic nanobody: A strategy for development of sensitive and green immunoassay for fumonisin B1. Talanta 2015, 143, pp.388-393. 
106.Li, C.; Mi, T.; Conti, G.O.; Yu, Q.; Wen, K.; Shen, J.; Ferrante, M.; Wang, Z. Development of screening fluorescence polarization immunoassay for the simultaneous detections of fumonisins B1 and B2 in maize. J Agric Food Chem 2015, 63(20), pp.4940-4946.

107.Ren, W.; Huang, Z.; Xu, Y.; Li, Y.; Ji, Y.; Su, B. Urchin-like gold nanoparticle-based immunochromatographic strip test for rapid detection of fumonisin $\mathrm{B}_{1}$ in grains. Anal BioanalChem 2015, 407(24), pp.7341-7348.

108.Lu, L.; Seenivasan, R.; Wang, Y.-C.; Yu, J.-H.; Gunasekaran, S. An electrochemical immunosensor for rapid sensitive detection of mycotoxins fumonisin B1 and deoxynivalenol. Electrochim Acta 2016, 213, pp.89-97.

109.Masikini, M.; Williams, A.R.; Sunday, C.E.; Waryo, T.T.; Nxusani, E.; Wilson, L.; Qakala, S. Bilibana, M.; Douman, S.; Jonnas, A.; Baker, P.G.L.; Iwuoha, E.I. Label free poly(2,5-dimethoxyaniline)-multiwalled carbon nanotubes impedimetric immunosensor for fumonisin B1 detection. Materials 2016, 9(4), pp.273-286.

110.Di Nardo, F.; Baggiani, C.; Giovannoli, C.; Spano, G.; and Anfossi, L. Multicolor immunochromatographic strip test based on gold nanoparticles for the determination of aflatoxin B1 and fumonisins. Microchim Acta 2017, 184(5), pp.1295-1304.

111.Urusov, A.E.; Petrakova, A.V.; Gubaydullina, M.K.; Zherdev, A. V.; Eremin, S. A.; Kong, D.; Liu, L.; Xu, C.; Dzantiev, B.B. High- sensitivity immunochromatographic assay for fumonisin B1 based on indirect antibody labelling. Biotechnol Lett 2017, 39(5), pp.751-758.

112.Bánati, H.; Darvas, B.; Fehér-Tóth, S.; Czéh, A.; Székacs, A. Determination of mycotoxin production of Fusarium species in genetically modified maize varieties by quantitative flow immunocytometry. Toxins 2017, 9(2), 70-81.

113.Tang, X.; Li, P.; Zhang, Z.; Zhang, Q.; Guo, J.; Zhang, W. An ultrasensitive gray-imaging- based quantitative immunochromatographic detection method for fumonisin B1 in agricultural products. Food Control 2017, 80, pp.333-340.

114.Peltomaa, R.; Benito-Peña, E.; Barderas, R.; Sauer, U.; González, M.; Moreno-Bondi, M.C. Microarraybased immunoassay with synthetic mimotopes for the detection of fumonisin B1. Anal Chem 2017, 89, pp.6216-6223.

115. Hao, K.; Suryoprabowo, S.; Hong, T.; Song, S.; Liu, L.; Zheng, Q.; Kuan, H. Immunochromatographic strip for ultrasensitive detection of fumonisin B1. Food and Agr Immunol 2018, 29(1), pp.699-710.

116.Anfossi, L.; Di Nardo, F.; Cavalera, S.; Giovannoli, C.; Spano, G.; Speranskaya, E.S.; Goryacheva, I.Y.; Baggiani, C. A lateral flow immunoassay for straightforward determination of fumonisin mycotoxins based on the quenching of the fluorescence of CdSe/ZnS quantum dots by gold and silver nanoparticles. Microchim Acta 2018, 185(2), 94.

117.Zhou,Y.; Huang. X.; Zhang, W.; Ji, Y.; Chen, R.; Xiong, Y. Multi-branched gold nanoflower-embedded iron porphyrin for colorimetric immunosensor. Biosens Bioelectro 2018, 102, pp.9-16.

118.Pagkali, V.; Petrou, P.S.; Makarona, E.; Peters, J.; Haasnoot, W.; Jobst, G.; Moser, I.; Gajos, K.; Budkowski, A.; Economou, A.; Misiakos, K.; Raptis, I.; Kakabakos, S.E. Simultaneous determination of aflatoxin B1, fumonisin B1 and deoxynivalenol in beer samples with a label-free monolithically integrated optoelectronic biosensor. J Hazard Mater 2018, 359, pp.445-453.

119.Peltomaa, R.; Amaro-Torres, F.; Carrasco, S.; Orellana, G.; Benito-Peña, E.; Moreno-Bondi, M.C. Homogeneous quenching immunoassay for fumonisin B1 based on gold nanoparticles and an epitopemimicking yellow fluorescent protein. ACS Nano 2018,12, pp.11333-11342. 
120.Yu, S.; He, L.; Yu, F.; Liu, L.; Qu, C.; Qu, L.; Liu, J.; Wu, Y.; Wu, Y. A lateral flow assay for simultaneous detection of Deoxynivalenol, Fumonisin B1 and Aflatoxin B1. Toxicon 2018, 156, pp.23-27.

121.Lu, T.; Zhan, S.; Zhou, Y.; Chen, X.; Huang, X.; Leng, Y.; Xiong, Y.; Xu, Y. Fluorescence ELISA based on CAT-regulated fluorescence quenching of CdTe QDs for sensitive detection of FB1. Anal Methods 2018, 10, pp.5797-5802.

122.Jie, M.; Yu, S.; Yu, F.; Liu, L.; He, L.; Li, Y.; Zhang, H.; Ou, L.; Harrington, P.; Wu, Y. An ultrasensitive chemiluminescence immunoassay for fumonisin B1 detections in cereals based on gold-coated magnetic nanoparticles. J Sci Food Agric 2018, 98(9), pp.3384-3390.

123.Li, Z.; Sheng, W.; Li, S.; Shi, Y.; Zhang, Y.; Wang, S. Development of a gold nanoparticle enhanced enzyme linked immunosorbent assay based on monoclonal antibodies for the detections of fumonisin B1, B2 and B3 in maize. Anal Methods 2018, 10, pp.3506-3513.

124.Chen, X.; Liang, Y.; Zhang, W.; Leng, Y.; Xiong, Y. A colorimetric immunoassay based on glucose oxidase-induced AuNP aggregation for the detection of fumonisin B1. Talanta 2018, 186, pp.29-35.

125.Sheng, W.; Wu, H.; Ji, W.; Li, Z.; Chu, F.; Wang, S. Visual non-instrumental on-site detection of fumonisin B1, B2, and B3 in cereal samples using a clean-up combined with gel-based immunoaffinity test column assay. Toxins 2018, 10(4), pp.165-179.

126.Wang, X.; Park, S.-G.; Ko, J.; Xiao, X.; Giannini, V.; Maier, S.A.; Kim, D.-H.; Choo, J. Sensitive and reproducible immunoassay of multiple mycotoxins using surface-enhanced Raman scattering mapping on 3D plasmonic nanopillar arrays. Small 2018, 14, pp.1801623.

127.Zhang, X.; Wang, Z.; Fang, Y.; Sun, R.; Cao, T.; Paudyal, N.; Fang, W.; Song, H. Antibody microarray immunoassay for simultaneous quantification of multiple mycotoxins in corn samples. Toxins 2018, 10(10), pp.415.

128.Shu, M.; Xu, Y.; Dong, J.; Zhong, C.; Hammock, B.D.; Wang, W.; Wu, G. Development of a noncompetitive idiometric nanobodies phage immumoassay for the determination of fumonisin B1. Food Agr Immunol 2019, 30(1), pp.510-521.

129.Lu, L.; Gunasekaran, S. Dual-channel ITO-microfluidic electrochemical immunosensor for simultaneous detection of two mycotoxins. Talanta 2019, 194, pp.709-716.

130.Qu, J.; Xie, H.; Zhang, S.; Luo, P.; Guo, P.; Chen, X.; Ke, Y.; Zhuang, J.; Zhou, F.; Jiang, W. Multiplex flow cytometric immunoassays for high-throughput screening of multiple mycotoxin residues in milk. Food Anal Methods 2019, 12, pp. 877-886.

131.Duan, H.; Li, Y.; Shao, Y.; Huang, X.; Xiong, Y. Multicolor quantum dot nanobeads for simultaneous multiplex immunochromatographic detection of mycotoxins in maize. Sens Actuators, B. Chemical 2019, 291, pp. 411-417.

132.Cheng, Z. X.; Ang, W. L.; Bonanni, A. Electroactive Nanocarbon Can Simultaneously Work as Platform and Signal Generator for Label-Free Immunosensing. ChemElectroChem 2019, 6(14), pp. 3615-3620.

133.Zhan, S.; Zheng, L.; Zhou, Y.; Wu, K.; Duan, H.; Huang, X.; Xiong, Y. A Gold Growth-Based Plasmonic ELISA for the Sensitive Detection of Fumonisin B1 in Maize. Toxins 2019, 11(6), pp. 323.

134.Shao, Y.; Duan, H.; Zhou, S.; Ma, T.; Guo, L.; Huang, X.; Xiong, Y. Biotin-Streptavidin SystemMediated Ratiometric Multiplex Immunochromatographic Assay for Simultaneous and Accurate Quantification of Three Mycotoxins. J Agric Food Chem 2019, 67(32), pp. 9022-9031.

135. Huang, X.; Huang, T.; Li, X.; Huang, Z. Flower-like gold nanoparticles-based immunochromatographic test strip for rapid simultaneous detection of fumonisin B1 and deoxynivalenol in Chinese traditional medicine. J Pharm Biomed Anal 2020, 177, pp. 112895. 
136.Hou, S.; Ma, J.; Cheng, Y.; Wang, H.; Sun, J.; Yan, Y. One-stop rapid detection of fumonisin B1, dexyonivalenol and zearalenone in grains. Food Control 2020, pp.107107._

137.Yang, H.; Zhang, Q.; Liu, X.; Yang, Y.; Yang, Y.; Liu, M.; Li, P.; Zhou, Y. Antibody-biotin-streptavidinhorseradish peroxidase (HRP) sensor for rapid and ultra-sensitive detection of fumonisins. Food Chem 2020, 316, pp.126356.

138.Ren, W.; Xu, Y.; Huang, Z.; Li, Y.; Tu, Z.; Zou, L.; He, Q.; Fu, J.; Liu, S.; Hammock, B. D. Single-chain variable fragment antibody-based immunochromatographic strip for rapid detection of fumonisin B1 in maize samples. Food Chem 2020, pp. 126546.

139.Liu, Z.; Hua, Q.; Wang, J.; Liang, Z.; Li, J.; Wu, J.; Shen, X.; Lei, H.; Li, X. A smartphone-based dual detection mode device integrated with two lateral flow immunoassays for multiplex mycotoxins in cereals. Biosens Bioelectron 2020, pp.112178.

140.Lee, K.M.; Herrman, T. Determination and prediction of fumonisin contamination in Maize by surfaceenhanced Raman spectroscopy (SERS). Food Bioprocess Technol 2016, 9(4), pp.588-603.

141.Smolinska-Kempisty, K.; Guerreiro, A.; Canfarotta, F.; Cáceres, C.; Whitcombe, M.J.; Piletsky, S. A comparison of the performance of molecularly imprinted polymer nanoparticles for small molecule targets and antibodies in the ELISA format. Sci Rep 2016, 6, pp. 37638.

142.Zhang, W.; Xiong, H.; Chen, M.; Zhang, X.; Wang, S. Surface-enhanced molecularly imprinted electrochemiluminescence sensor based on Ru@SiO2 for ultrasensitive detection of fumonisin B1. Biosens Bioelectron 2017, 96, pp. 55-61.

143.Munawar, H.; Smolinska-Kempisty, K.; Cruz, A.G.; Canfarotta, F.; Piletska, E.; Karim, K.; Piletsky, S.A. Molecular imprinted polymer nanoparticle-based assay (MINA): application for fumonisin B1 determination. Analyst 2018,143, pp.3481-3488.

144.Mao. L.; Ji, K.; Yao, L.; Xue, X.; Wen, W.; Zhang, X.; Wang, S. Molecularly imprinted photoelectrochemical sensor for fumonisin B1 based on GO-CdS heterojunction. Biosens Bioelectron 2019, 127, pp. 57-63.

145.Munawar, H.; Safaryan, A. H.; De Girolamo, A.; Garcia-Cruz, A.; Marote, P.; Karim, K.; Lippolis, V.; Pascale, M.; Piletsky, S. A. Determination of Fumonisin B1 in maize using molecularly imprinted polymer nanoparticles-based assay. Food Chem 2019, 298, 125044.

146. Chotchuang, T.; Cheewasedtham, W.; Jayeoye, T. J.; Rujiralai, T. Colorimetric determination of fumonisin B1 based on the aggregation of cysteamine-functionalized gold nanoparticles induced by a product of its hydrolysis. Microchim Acta 2019, 186(9), pp.655.

147.Li, L.; Chen, W.; Li, H.; Iqbal, J.; Zhu, Y.; Wu, T.; Du, Y. Rapid determination of fumonisin (FB1) by syringe SPE coupled with solid-phase fluorescence spectrometry. SAA 2020, 226, pp.117549.

148.McKeague, M.; Bradley, C.R.; De Girolamo, A.; Visconti, A.; Miller, J.D.; DeRosa, M.C. Screening and initial binding assessment of fumonisin B1 aptamers. Int J Mol Sci 2010, 11(12), pp.4864-4881.

149.Chen, X.; Huang, Y.; Duan, N.; Wu, S.; Xia, Y.; Ma, X.; Zhu, C.; Jiang, Y.; Ding, Z.; Wang, Z. Selection and characterization of single stranded DNA aptamers recognizing fumonisin B1. Microchim Acta 2014, 181, pp.1317-1324.

150.Frost, N.R.; McKeague, M.; Falcioni, D.; DeRosa, M.C. An in solution assay for interrogation of affinity and rational minimer design for small molecules binding aptamers. Analyst 2015, 140, pp.6643-6651.

151.Wu, S.; Duan, N.; Ma, X.; Xia, Y.; Wang, H.; Wang, Z.; Zhang, Q. Multiplexed fluorescence resonance energy transfer aptasensor between upconversion nanoparticles and graphene oxide for the simultaneous determination of mycotoxins. Anal Chem 2012, 84(14), pp.6263-6270. 
152.Wu, S.; Duan, N.; Li X.; Tan, G.; Ma, X.; Xia, Y.; Wang, Z.; Wang, H. Homogenous detection of fumonisin $\mathrm{B}_{1}$ with a molecular beacon based on fluorescence energy transfer between NaYF 4 :YB, Ho upconversion nanoparticles and gold nanoparticles. Talanta 2013, 116(15), pp.611-618.

153.Wang, W.F.; Wu, S.; Ma, X.Y.; Xia, Y.; Wang, Z.P. Novel methods for fumonisin B1 detection based on AuNPs labelling and aptamer recognition. J Food Sci Biotech 2013, 32(5), pp.501-508.

154.Yue, S.; Jie, X.; Wei, L.; Bin, C.; Dou, W.D.; Yi, Y., QingXia, L.; JianLin, L.; TieSong, Z. Simultaneous detection of ochratoxin $A$ and fumonisin $B 1$ in cereal samples using an aptamer-photonic crystal encoded suspension array. Anal Chem 2014, 86(23), pp.11797-11802.

155.Zhao, Y.; Luo, Y.; Li, T.; Song, Q. AuNPs driven electrochemiluminescence aptasensors for sensitive detection of fumonisin B1. RSC Advances 2014, 4, pp.57709-57714.

156.Chen, X.; Huang, Y.; Ma, X.; Jia, F.; Guo, X.; Wang, Z. Impedimetric aptamer-based determination of the mold toxin fumonisin $B_{1}$. Microchim Acta 2015, 182(9-10), pp.1709-1714.

157.Chen, X.; Bai, X.; Li, H.; Zhang, B. Aptamer-based microcantilever array biosensor for detection of fumonisin $\mathrm{B}_{1}$. RSC Advances 2015, 5, pp.35448-35452.

158.Shi, Z.-Y.; Zheng Y.-T.; Zhang, H.-B.; He, C.-H.; Wu, W.-D.; Zhang, H.-B. DNA electrochemical aptasensor for detecting fumonisin B1 based on graphene and thionine nanocomposite. Electroanalysis 2015, 27(5), pp.1097-1103.

159.Gui, H.; Jin, Q.; Zhang, Y.; Wang, X.; Yang, Y.; Shao, C.; Cheng, C.; Wei, F.; Yang, Y.; Yang, M.; Song, $\mathrm{H}$. Development of an aptamer/ fluorescence dye PicoGreen-based method for detection of fumonisin B1. Sheng Wu Gong Cheng Xue Bao 2015, 31(9), pp.1393-1400.

160.Ren, C.; Li, H.; Lu, X.; Quian, J.; Zhu, M.; Chen, W.; Liu, Q.; Hao, N; Li, H.; Wang, K. A disposable aptasensing device for label-free detection of fumonisin B1 by integrating PDMS film-based micro-cell and screen-printed carbon electrode. Sens Actuators, B 2017, 251, pp.192-199.

161.Yang, Y.; Li, W.; Shen, P.; Liu, R.; Li, Y.; Xu, J.; Zheng, Q.; Zhang, Y.; Li, J.; Zheng. T. Aptamer fluorescence signal recovery screening for multiplex mycotoxins in cereal samples based on photonic crystal microsphere suspension array. Sens Actuators, B 2017, 248, pp.351.358.

162.Wang, C.; Qian, J.; An, K.; Huang, X.; Zhao, L.; Liu, Q.; Hao, N.; Wang, K. Magneto-controlled aptasensor for simultaneous electrochemical detection of dual mycotoxins in maize using metal sulfide quantum dots coated silica as labels. Biosens Bioelectron 2017, 89, pp.802-809.

163.Molinero-Fernández, A.; Moreno-Guzmán, M.; Ángel López, M.; Escarpa, A. Biosensing strategy for simultaneous and accurate quantitative analysis of mycotoxins in food samples using unmodified graphene micromotors. Anal Chem 2017, 89, pp.10850-10857.

164.Tian, H.; Sofer, Z.; Pumera, M.; Bonanni, A. Investigation on the ability of heteroatom-doped graphen for biorecognition. Nanoscale 2017, 9, pp.3530-3536.

165.王红旗, 王俊艳, 洪慧杰, 尹海燕, Maragos, C., 张 玲, 刘继红.伏马毒素 B1 核酸适配体链置换探针的 笁选及应用. 农产品质量与安全 2017,1, pp.44-48.

166.Liu, R.; Li, W.; Cai, T.; Deng, Y.; Ding, Z.; Liu, Y.; Zhu, X.; Wang, X.; Liu, J.; Liang, B.; Zheng, T.; LI, J. $\mathrm{TiO}_{2}$ nanolayer-enhanced fluorescence for simultaneous multiplex mycotoxin detection by aptamer microarrays on a porous silicon surface. ACS Appl Mater Interfaces 2018, 10, pp.14447-14453.

167.Cheng, Z.; Bonanni, A. All-in-One: Electroactive nanocarbon as simultaneous platform and label for single-step biosensing. Nanomaterials 2018, 24, pp.6380-6385. 
168.Molinero-Fernández, A.; Jodra, A.; Moreno-Guzmán, M.; López, M.A.; Escarpa, A. Magnetic reduced graphene oxide/nickel/platinum nanoparticles micromotors for mycotoxin analysis. Chem Eur $\mathrm{J} 2018$, 24, pp.7172-7176.

169.Hao, N.; Lu, J.; Zhou, Z.; Hua, R.; Wang, K. A pH-resolved colorimetric biosensor for simultaneous multiple target detection. ACS Sensors 2018, 3, pp.2159-2165.

170.Niazi, S.; Khan, I.M.; Yan, L.; Khan, M.I.; Mohsin, A.; Duan, N.; Wu, S.; Wang, Z. Simultaneous detection of fumonisin $\mathrm{B} 1$ and ochratoxin $\mathrm{A}$ using dual-color, time-resolved luminescent nanoparticles $\left(\mathrm{NaYF}_{4}\right.$ $\mathrm{Ce}, \mathrm{Tb}$ and $\mathrm{NH}_{2}-\mathrm{Eu} / \mathrm{DPA} @ \mathrm{SiO}_{2}$ ) as labels. Anal BioanalChem 2019; 411, pp.1453-1465.

171.Wang, C.; Huang, X.; Tian, X.; Zhang, X.; Yu, S.; Chang, X.; Ren, Y.; Qian, J. A multiplexed FRET aptasensor for the simultaneous detection of mycotoxins with magnetically controlled graphene oxide/Fe3O4 as a single energy acceptor. Analyst 2019, 144(20), 6004-6010.

172. Wei, M.; Zhao, F.; Feng, S.; Jin, H. A novel electrochemical aptasensor for fumonisin B 1 determination using DNA and exonuclease-I as signal amplification strategy. BMC Chemistry 2019, 13(1), pp.1-6.

173.Han, Z.; Tang, Z.; Jiang, K.; Huang, Q.; Meng, J.; Nie, D.; Zhao, Z. Dual-target electrochemical aptasensor based on co-reduced molybdenum disulfide and Au NPs (rMoS2-Au) for multiplex detection of mycotoxins. Biosens Bioelectron 2020, 150, pp.11894.

174.Wei, M.; Xin, L.; Feng, S.; Liu, Y. Simultaneous electrochemical determination of ochratoxin A and fumonisin B1 with an aptasensor based on the use of a $\mathrm{Y}$-shaped DNA structure on gold nanorods. Microchim Acta 2020, 187(2), pp.1-7.

175.He, D.; Wu, Z.; Cui, B.; Xu, E. Aptamer and gold nanorod-based fumonisin B1 assay using both fluorometry and SERS. Microchim Acta 2020, 187(4), pp.1-8.

176. Tao, Z.; Zhou, Y.; Li, X.; Wang, Z. Competitive HRP-Linked Colorimetric Aptasensor for the Detection of Fumonisin B1 in Food based on Dual Biotin-Streptavidin Interaction. Biosensors 2020, 10(4), pp.31.

177.He, D.; Wu, Z.; Cui, B.; Jin, Z.; Xu, E. A fluorometric method for aptamer-based simultaneous determination of two kinds of the fusarium mycotoxins zearalenone and fumonisin B1 making use of gold nanorods and upconversion nanoparticles. Microchim Acta 2020, 187, pp.254.

178. Kesici, E.; Erdem, A. Impedimetric detection of Fumonisin B1 and its biointeraction with fsDNA. Int $J$ Biol Macromol 2019, 139, pp.

179.Siler, D.J.; Gilchrist, D.G. Determination of host-selective phytotoxins from Alternaria alternata f.sp. lycopersici as their maleyl derivatives by high-performance liquid chromatography. J Chromatogr 1982 , 238, pp.167-173.

180.Sydenham, E.W.; Gelderblom, W.C.A.; Thiel, P.G.; Marasas, W.F.O. Evidence for the natural occurrence of fumonisin B1, a mycotoxin produced by Fusarium moniliforme, in corn. J Agric Food Chem 1990, 38(1), pp.285-290.

181.Shepard, G.S.; Sydenham, E.W.; Thiel, P.G.; Gelderblom, C.A. Quantitative determination of fumonisins B1 and B2 by high-performance liquid chromatography with fluorescence detection. J Liq Chromatogr 1990, 13(10), pp.2077-2087.

182.Bordin, K.; Rosim, R.E.; Neeff, D.V.; Rottinghaus, G.E.; Oliveira, C.A.F. Assessment of dietary intake of fumonisin B1 in São Paulo, Brazil. Food Chem 2014, 155, pp.174-178.

183. Holcomb, M.; Thompson, H.C.; Hankins, L.J. Analysis of fumonisin B1 in rodent feed by gradient elution HPLC using precolumn derivatization with FMOC and fluorescence detection. J Agric Food Chem 1993, 41(5), pp.764-767. 
184.Food Standards Agency. 2019. Incidents annual reports 2006-2017. Available online: https://www.food.gov.uk/about-us/reports-and-accounts (Accessed on 10 April 2020).

185. Gilbert-Sandoval, I.; Wesseling, S.; Rietjens, I.M. Occurrence and probabilistic risk assessment of fumonisin B1, fumonisin B2 and deoxynivalenol in nixtamalized maize in Mexico City. Toxins 2020, 12(10), p.644.

186.Yapo, A.E.; Strub, C.; Durand, N.; Ahoua, A.R.C.; Schorr-Galindo, S.; Bonfoh, B.; Fontana, A.; Koussémon, M. Mass spectrometry-based detection and risk assessment of mycotoxin contamination of 'kankankan'used for roasted meat consumption in Abidjan, Côte d'Ivoire. Food Addit Contam: Part A 2020, 37(9), pp.1564-1578.

187.Sulyok, M.; Krska, R.; Senyuva, H. Profiles of fungal metabolites including regulated mycotoxins in individual dried Turkish figs by LC-MS/MS. Mycotoxin Res 2020, 36(4), pp.381-387.

188.Zhang, L.; Sun, Y.; Liang, X.; Yang, Y.; Meng, X.; Zhang, Q.; Li, P.; Zhou, Y. Cysteamine triggered "turn-on" fluorescence sensor for total detection of fumonisin B1, B2 and B3. Food Chem 2020, 327, pp.127058.

189.Hou, S.; Ma, J.; Cheng, Y.; Wang, H.; Sun, J.; Yan, Y. Quantum dot nanobead-based fluorescent immunochromatographic assay for simultaneous quantitative detection of fumonisin B1, dexyonivalenol, and zearalenone in grains. Food Control 2020, 117, pp.107331.

190.Guo, L.; Wang, Z.; Xu, X.; Xu, L.; Kuang, H.; Xiao, J.; Xu, C. Europium nanosphere-based fluorescence strip sensor for ultrasensitive and quantitative determination of fumonisin B 1. Anal Methods 2020, 12, pp.5229-5235.

191.Munawar, H.; Garcia-Cruz, A.; Majewska, M.; Karim, K.; Kutner, W.; Piletsky, S.A., Electrochemical determination of fumonisin B1 using a chemosensor with a recognition unit comprising molecularly imprinted polymer nanoparticles. Sens Actuators, B 2020, 321, pp.128552.

192.Hines, H.B.; Brueggemann, E.E.; Holcomb, M.; Holder, C.L. Fumonisin B1 analysis with capillary electrophoresis-electrospray ionization mass spectrometry. Rapid Commun Mass Spectrom 1995, 9(6), pp.519-524.

193. Holcomb, M., Thompson Jr, H.C. Analysis of fumonisin B1 in corn by capillary electrophoresis with fluorescence detection of the FMOC derivative. J Microcolumn Sep 1995, 7(5), pp.451-454.

194. Maragos, C.M. Capillary zone electrophoresis and HPLC for the analysis of fluorescein isothiocyanatelabeled fumonisin B1. Journal of Agricultural and Food Chem 1995, 43(2), pp.390-394.

195. Maragos, C.M. Detection of the mycotoxin fumonisin B1 by a combination of immunofluorescence and capillary electrophoresis. Food and Agr Immunol 1997, 9(3), pp.147-157.

196.Kecskeméti, Á.; Nagy, C.; Biró, P.; Szabó, Z.; Pócsi, I.; Bartók, T.; Gáspár, A. Analysis of fumonisin mycotoxins with capillary electrophoresis-mass spectrometry. Food Addit Contam: Part A 2020, 37(9), pp.1553-1563.

197.Jiang, D.; Huang, C.; Shao, L.; Wang, X.; Jiao, Y.; Li, W.; Chen, J.; Xu, X. Magneto-controlled aptasensor for simultaneous detection of ochratoxin $A$ and fumonisin B1 using inductively coupled plasma mass spectrometry with multiple metal nanoparticles as element labels. Anal Chim Acta 2020, 1127, pp.182-189.

198.Wu, Z.; He, D.; Cui, B.; Jin, Z.; Xu, E.; Yuan, C.; Liu, P.; Fang, Y.; Chai, Q. Trimer-based aptasensor for simultaneous determination of multiple mycotoxins using SERS and fluorimetry. Microchim Acta 2020, 187(9), pp.1-7. 
199.Zheng, Y.T.; Zhao, B.S.; Zhang, H.B.; Jia, H.; Wu, M. Colorimetric aptasensor for fumonisin B1 detection by regulating the amount of bubbles in closed bipolar platform. J Electroanal Chem 2020, 877, p.114584.

200.Mirón-Mérida, V.A.; González-Espinosa, Y.; Gong, Y.Y.; Guo, Y.; Goycoolea, F.M. Comparison of the Performances of Two Aptamers on a Colorimetric Assay for the Quantification of Fumonisin B1. MDPI Proceedings 2020, 60(1), pp.19. 